\title{
Técnicas de clusterização e estratificação de indivíduos para estudo de redes funcionais cerebrais
}

\author{
Taiane Coelho Ramos
}

\author{
TESE APRESENTADA AO \\ Instituto DE MATEMÁticA E EstatísticA \\ dA Universidade de São Paulo \\ PARA OBTENÇÃO DO TÍTULO DE \\ DOUTORA EM CIÊNCIAS
}

Programa: Ciência da Computação

Orientador: Prof. Dr. André Fujita

Durante o desenvolvimento deste trabalho o autor recebeu auxílio financeiro do CNPq (Processo 141492/2017-1)

São Paulo

19 de março de 2021 



\title{
Técnicas de clusterização e estratificação de indivíduos para estudo de redes funcionais cerebrais
}

\author{
Taiane Coelho Ramos
}

Esta versão da tese contém as correções e alterações sugeridas pela Comissão Julgadora

durante a defesa da versão original do trabalho, realizada em 19 de março de 2021.

Uma cópia da versão original está disponível no Instituto de Matemática e Estatística da Universidade de São Paulo.

Comissão Julgadora:

- Prof. Dr. André Fujita (orientador) - IME-USP

- Prof. Dr. Luiz Antonio Baccalá - EP-USP

- Profa. Dra. Leticia de Oliveira - UFF

- Prof. Dr. Daniel Yasumasa Takahashi - UFRN

- Prof. Dr. João Ricardo Sato - UFABC 
Autorizo a reprodução e divulgação total ou parcial deste trabalho, por qualquer meio convencional ou eletrônico, para fins de estudo e pesquisa, desde que citada a fonte. 
Quando partires de regresso à Ítaca, faz votos de que seja longa a viagem, plena de aventuras e de experiências. [...] Por pobre que a descubras, Ítaca não te traiu. Sábio como és agora, senhor de tanta experiência, terás compreendido o sentido de Ítaca.

- Konstantínos Kaváfis 



\section{Agradecimentos}

Este trabalho não seria possível sem o apoio, horas de trabalho e dedicação de diversas pessoas. Listo aqui algumas dessas pessoas que tiveram fundamental importância nesta jornada.

Primeiramente agradeço ao meu orientador, Prof. André Fujita, que me aceitou como sua aluna de mestrado e doutorado no programa de ciência da computação do IME-USP. Agradeço por sua disponibilidade e pronto atendimento todas as vezes que precisei tirar dúvidas, pedir conselhos ou ter trabalhos revisados. Foram muitas horas de dedicação e atenção a este trabalho.

Agradeço à Profa. Janaína Mourão-Miranda por me receber em seu laboratório na UCL e a toda a sua equipe. Agradeço ao Agoston, Fabio e Rick por todo o tempo dedicado a me auxiliar com as ferramentas e discutir resultados. Agradeço também a Cemre, Konstantino, Najiba, Nils e James pelas boas conversas e trocas de experiências. Em especial agradeço à Profa. Isabel que se tornou uma querida amiga e me apoiou muito com toda sua compreensão e acolhimento.

Meu tempo na Inglaterra me trouxe muito mais do que experiências profissionais, mas também bons amigos que foram fundamentais para manter meu foco e saúde mental. Agradeço a todas as pausas para o chá com Rica, Eleni, Ann e Fotini. Todas as conversas de almoço com Kiko, Michelli, Bepe, Pera e Sean. Todos do CMIC e AI Center que foram sempre tão gentis, me convidando para almoçar, me ensinando a usar a máquina de café e conversando comigo nas confraternizações. Em especial, fui muito feliz por conhecer a Burcu, minha irmã (de alma) turca, que se manteve estudando comigo todos os dias, mesmo à distância, para nos apoiarmos e contarmos nossos "pomodoros" juntas.

No meu laboratório na USP também pude encontrar bastante companheirismo de meus colegas. Agradeço a todos que participaram das discussões do grupo de estudos de estatística em grafos (GEEG). Agradeço especialmente à Suzana, que sempre me foi tão companheira e me auxiliou em todas as horas que precisei. Agradeço também ao Grover, Eduardo, Maciel, Diogo e Vinícius pelos materiais de estudo e discussões. 
Também gostaria de agradecer a todos do Cursinho Cardume da Unifesp Santos, que me acolheu por 5 anos como educadora voluntária de matemática. Manter a atividade de ensino me ajudou a relembrar constantemente minha motivação para perseguir uma carreira acadêmica. Sou grata a tudo o que construímos neste cursinho e vou levar os ensinamentos para toda a vida.

Por fim, agradeço à minha família, que apesar de não compreender totalmente meus motivos para seguir nesta carreira tão difícil e com tantos desafios, nunca me pediu para desistir. Agradeço ao meu pai que me estimulou a assistir "O mundo de Beakman" quando eu tinha 4 anos e, sem saber, despertou meu interesse pela ciência. Agradeço à minha mãe que escolheu me matricular em uma boa escola e em um bom curso de inglês. Meus mais de 10 anos de curso foram bem utilizados. Agradeço aos meus irmãos Milton e Danilo por sempre terem me feito tantas perguntas, o que me forçou a saber sempre mais. Agradeço também à minha irmã Yasmin, que me foi boa companhia na infância e (sem querer) me mostrou que está tudo bem se a gente precisar desistir e voltar para casa. Agradeço também a minha madrinha Márcia, por todas as horas de conversas. E não poderia deixar de agradecer ao Carlos, pelo suporte que me deu durante os 4 anos deste doutorado. 


\section{Resumo}

Taiane Coelho Ramos. Técnicas de clusterização e estratificação de indivíduos para estudo de redes funcionais cerebrais. Tese (Doutorado). Instituto de Matemática e Estatística, Universidade de São Paulo, São Paulo, 2021.

Em diversas aplicações de neurociência gostaríamos de entender semelhanças e diferenças entre indivíduos analisando as conectividades do cérebro. Para isso, propomos duas abordagens: (i) agrupar indivíduos semelhantes ou (ii) posicioná-los em um espectro estratificado de um diagnóstico. Para (i), nós modelamos as redes cerebrais como um grafo e apresentamos dois métodos de clusterização baseados em análise espectral: um k-means para grafos do mesmo tamanho e uma abordagem baseada em modelo, chamada gCEM, para grafos de tamanhos diferentes. Para avaliar os pontos fortes e fracos dos métodos, projetamos alguns cenários usando modelos de grafos aleatórios. Também aplicamos a dados reais de compostos químicos e de redes cerebrais funcionais. Para (ii) nós utilizamos uma análise multivariada para encontrar associações cérebro-comportamentais e criar uma estratificação dos indivíduos variando de típicos a atípicos. Aplicamos um framework de múltiplos holdouts com o método Sparse Partial Least Squares para encontrar essas associações. Utilizamos uma amostra de 349 crianças e adolescentes do conjunto de dados ABIDE II e encontramos um modo de associação significativo entre dados fMRI e características comportamentais. As variáveis comportamentais mais importantes nesta associação estão relacionadas à capacidade de resposta social e as conexões cerebrais mais importantes estão dentro da rede de modo padrão e entre as redes somatomotora e visual.

Palavras-chave: Agrupamento de grafos. Densidade espectral. Grafos aleatórios. SPLS. Associações cérebro-comportamentais. 



\begin{abstract}
Taiane Coelho Ramos. Clustering techniques and stratification of individuals to study functional brain networks. Thesis (Doctorate). Institute of Mathematics and Statistics, University of São Paulo, São Paulo, 2021.
\end{abstract}

In several neuroscience applications, we would like to understand the similarities and the differences between individuals in the same group by analysing their brain connectivity. For this, we propose two approaches: (i) to group similar individuals or (ii) to place them in a stratified spectrum of a diagnosis. For (i), we model the brain networks as a graph and present two clustering methods based on spectral analysis: a k-means for graphs of the same size and a model-based approach, called gCEM, for graphs of different sizes. To assess the strengths and weaknesses of the methods, we designed some scenarios using random graph models. We also apply it to real data on chemical compounds and functional brain networks. For (ii), we use a multivariate analysis to find brain-behavioural associations and create stratification of individuals ranging from typical to atypical. We applied a framework of multiple holdouts with Sparse Partial Least Squares method to find these associations. We used a sample of 349 children and adolescents from the ABIDE II data set and found a significant association between fMRI data and behavioural characteristics. The most important behavioural variables in this association are related to social responsiveness. The most important brain connections are within the default-mode network and between the somatomotor and visual networks.

Keywords: Graph clustering. Spectral density. Random Graph. SPLS. Brain-Behaviour associations. 



\section{Lista de Abreviaturas}

CEM Clusterização por Expectativa-Maximização

(Clustering Expectation-Maximization)

ECoG Eletrocorticografia

ER Modelo de Grafos Aleatórios de Erdös-Rényi

FBN Rede Funcional do Cérebro (Functional Brain Network)

gCEM Clusterização por Expectativa-Maximização de Grafos

GRG Modelo de grafos aleatórios Geométrico (Geometric Random Graph)

JS Divergência de Jensen-Shannon

KL Divergência de Kullback-Leibler

KR Modelo de Grafos Aleatórios K-regular

LOC Perda de Consciência (Loss of Consciousness)

MIX Mistura de Modelos de Grafos Aleatórios

PA Modelo de Grafos Aleatórios com Ligação Preferencial (Preferential Attachment)

WS Modelo de Grafos Aleatórios de Watts-Strogatz

TEA Transtorno do Espectro do Autismo

PDD-NOS Transtorno Global do Desenvolvimento Sem Outra Especificação

DSM-5 Manual Diagnóstico e Estatístico de Transtornos Mentais quinta edição

CID-10 Classificação Internacional de Doenças décima edição

RDoC Critérios de Domínio de Pesquisa (Research Domain Criteria)

SPLS Sparse Partial Least Squares

rs-fMRI Imagem de Ressonância Magnética Funcional em Estado de Repouso

SRS Social Responsiveness Scale

CBCL Child Behavior Checklist

BRIEF Behavior Rating Inventory of Executive Function

DT Desenvolvimento Típico

ABIDE Autism Brain Imaging Data Exchange

AFNI Analysis of Functional NeuroImages 
FSL fMRIB Software Library

NITRC NeuroImaging Tools and Resources Collaboratory

MNI Montreal Neurological Institute

FD Framewise Displacement

DVARS Derivada da Variância quadrada média sobre voxels

ROI Região de interesse (Region of Interest)

SPLS Sparse Partial Least Squares 


\section{Lista de Figuras}

2.1 Esquemático da clusterização de grafos. . . . . . . . . . . . . . . . 6

2.2 Resultados da clusterização da simulação 1. . . . . . . . . . . . . . . . . . 17

2.3 Representação gráfica e resultados da simulação 2 . . . . . . . . . . . . . 19

2.4 Resultados da clusterização da simulação 3. . . . . . . . . . . . . . . . 20

2.5 Resultados da aplicação em redes funcionais do cérebro. . . . . . . . . . . 21

2.6 Repetição da análise apresentada em 2.5 com outros valores de corte. . . 22

2.7 Avaliação do ajuste do modelo selecionado aos grafos de compostos químicos. 24

3.1 Visão geral do modelo Sparse Partial Least Squares (SPLS) . . . . . . . . . 34

3.2 Representação da divisão dos dados em conjunto otimização e holdout. 37

3.3 Representação das divisões dos dados para otimizar os parâmetros de regularização. . . . . . . . . . . . . . . . . 38

3.4 Representação do teste de permutação para avaliação estatística do modelo

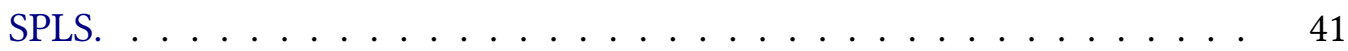

3.5 Relação entre a associação cérebro-comportamental e o diagnóstico. . . . 43

3.6 Coeficientes de correlação das variáveis comportamentais para a associação cérebro-comportamental encontrada. . . . . . . . . . . . . . 44

3.7 Pesos das variáveis de comportamento selecionadas pelo SPLS. . . . . . . 45

3.8 Representação da variação da conectividade cerebral. . . . . . . . . . . . 46

3.9 Comparação da conectividade funcional entre redes. . . . . . . . . . . . . 47 



\section{Sumário}

1 Introdução 1

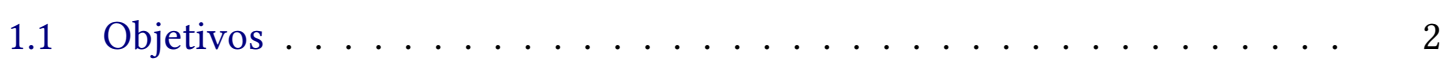

2 Clusterização de indivíduos com base em conectividades funcionais cerebrais 5

2.1 Clusterização de grafos . . . . . . . . . . . . . . . . . . 5

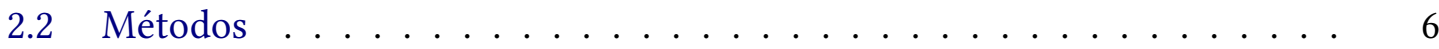

2.2.1 Densidade espectral de grafos . . . . . . . . . . . 6

2.2.2 A estatística de silhueta . . . . . . . . . . . . . 8

$2.2 .3 \quad$ K-means . . . . . . . . . . . . . . . . . . . 8

2.2.4 Algoritmo de clusterização baseado em modelo . . . . . . . . . . 9

2.2.5 Modelos de grafos aleatórios . . . . . . . . . . . . . . . . 12

2.2.6 Algoritmo de troca aleatória de Markov . . . . . . . . . . . . 13

2.2 .7 Simulações . . . . . . . . . . . . . . . . . . . . . . 14

2.2 .8 Redes do mundo real . . . . . . . . . . . . . . . . . . 15

2.2.9 Avaliação de desempenho da clusterização . . . . . . . . . . . . . 16

2.3 Resultados e discussão . . . . . . . . . . . . . . . . . . . . . . 16

2.3.1 Aplicações em dados simulados . . . . . . . . . . . . . . . . 16

2.3.2 Aplicações em dados empíricos . . . . . . . . . . . . . . . 18

2.4 Conclusão . . . . . . . . . . . . . . . . . . . . . . . . . 24

3 Estratificação do espectro autista com base em associações cérebro$\begin{array}{ll}\text { comportamentais } & 27\end{array}$

3.1 Associações cérebro-comportamentais em autismo . . . . . . . . . . . . 27

3.2 Métodos . . . . . . . . . . . . . . . . . . . . 29

$3.2 .1 \quad$ Dados . . . . . . . . . . . . . . . . . . . . . 29

3.2.2 Pré-processamento dos dados de fMRI . . . . . . . . . . . . . 29

3.2.3 Redes de conectividade funcional . . . . . . . . . . . . . . . 30

3.2.4 Dados comportamentais e demográficos . . . . . . . . . . . . . 31 
3.2.5 Remoção de covariáveis e normalização dos dados . . . . . . . . 33

3.2.6 Modelagem SPLS dos dados cerebrais e comportamentais . . . . 33

3.2.7 O método Sparse Partial Least Squares (SPLS) . . . . . . . . . . . 34

3.2.8 Framework de múltiplos holdouts . . . . . . . . . . . . . 36

3.3 Resultados . . . . . . . . . . . . . . . . . . . . . 42

3.4 Discussão . . . . . . . . . . . . . . . . . . . . . . . 48

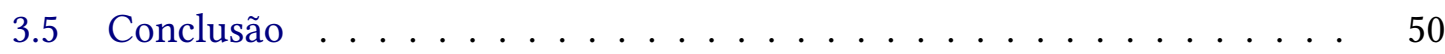

4 Considerações finais

5 Contribuições $\quad 53$

5.1 Produção de textos técnicos e científicos . . . . . . . . . . . . . 53

5.1 .1 Artigo publicado . . . . . . . . . . . . . . . . 53

5.1 .2 Artigo submetido . . . . . . . . . . . . . 53

5.1 .3 Artigos em preparação . . . . . . . . . . . . . . 53

5.2 Software ............................ 54

Referências 


\section{Capítulo 1}

\section{Introdução}

Sabemos que o cérebro é formado por redes de neurônios interconectados. Estudos já de longa data têm elucidado características estruturais do cérebro, como os diferentes tipos de neurônios, identificação de circuitos neurais e fibras de longa distância conectando diferentes regiões cerebrais. Novas técnicas de neuroimagem não invasiva (FrISTON, 2009) tem nos auxiliado a entender não só a organização estrutural, mas também nos possibilitam coletar dados sobre o funcionamento do cérebro. Utilizando técnicas de neuroimagem ou de eletroencefalografia, capturamos o sinal de pequenas regiões do cérebro e utilizamos métodos estatísticos para inferir uma rede funcional relacionando regiões que parecem se ativar conjuntamente (S. M. SMITH, 2012). Este tipo de rede pode ser construída tanto a partir do cérebro em estado de repouso (resting-state) (Fox e RAICHLE, 2007) como durante a execução de uma tarefa (task-evoked) (Allen et al., 2004). As redes funcionais nos auxiliam a entender melhor o funcionamento do cérebro e sua relação com a organização estrutural.

A neurociência visa não só entender a estrutura e funcionamento do cérebro, mas aplicar este conhecimento para entender melhor diferenças entre redes cerebrais de diferentes grupos. Por exemplo, entender diferenças na conectividade cerebral do indivíduo acordado ou sob anestesia, ou entre pessoas com e sem transtornos mentais (UHLHAAS e Singer, 2012). Diferenciar as redes dos grupos nos possibilita entender as mudanças causadas na rede cerebral. Uma das possibilidades para diferenciar os grupos é utilizar uma abordagem de clusterização, em que formamos grupos com os indivíduos mais próximos entre si. Outra possibilidade seria utilizar um método de estratificação em que identificamos uma dimensão de variação dos dados e os indivíduos são posicionados em um espectro de variação das características avaliadas.

A clusterização é um processo de classificação não-supervisionado que forma grupos de acordo com uma medida de distância. Para isso, vamos primeiramente modelar as redes funcionais como grafos. Então, propomos um algoritmo de clusterização para grafos do mesmo tamanho baseado no algoritmo k-means (MACQUEEN et al., 1967; LLOYd, 1982), considerando como distância a divergência de Jensen-Shannon (JS) entre os espectros de dois grafos. Em seguida, propomos um algoritmo de clusterização para grafos de tamanhos diferentes baseado no algoritmo de clusterização por Expectativa-Maximização (CEM) (Celeux e Govaert, 1992; Celeux e Govaert, 1995) que se baseia na divergência de 
Kullback-Leibler (KL) entre o grafo e um modelo de grafos aleatórios para definir os clusters. Avaliamos os algoritmos desenvolvidos em dados simulados. Também aplicamos os algoritmos em dados de eletrocorticografia (ECoG) de um macaco (NAGASAKA et al., 2011) para separar as redes funcionais do cérebro acordado e sob anestesia. Por fim, aplicamos para separar dois tipos de compostos químicos com redes de tamanhos diferentes.

A estratificação dos dados em um espectro de variação da característica pode ser feita utilizando métodos que identificam dimensões de variações dos dados. Com técnicas multivariadas é possível relacionar mais de uma informação sobre os indivíduos, como os dados de ressonância magnética funcional e escalas psicométricas (S. SMITH et al., 2015; Drysdale et al., 2017; Mihalik, Ferreira, Rosa et al., 2019). Neste trabalho, utilizamos o método Sparse Partial Least Squares (SPLS) (Monteiro, Rao, Shawe-Taylor et al., 2016) para identificar uma associação entre cérebro e comportamento de indivíduos com desenvolvimento típico (DT) e com transtorno do espectro do autismo (TEA) de forma a maximizar a covariância entre essas modalidades de dados. O SPLS atribui pesos para as variáveis que nos permite avaliar o sentido da variação na associação (aumento ou diminuição de conectividade ou de característica comportamental) e um score em relação a cada modalidade de variável (cérebro e comportamento) para cada indivíduo. Os scores identificam a variação das características de cada indivíduo de forma que, ao plotarmos todos os indivíduos representados por seus scores podemos visualizar o espectro de variação das características de autismo no grupo de estudo. Para avaliar os resultados utilizamos uma abordagem de treino e teste em conjuntos independentes. Para aumentar a chance de termos resultados generalizáveis, nós utilizamos um framework de múltiplos holdouts proposto por Mihalik, Ferreira, Moutoussis et al., 2020.

O objetivo deste trabalho é apresentar duas estratégias para auxiliar o estudo de redes funcionais do cérebro. A primeira em forma de algoritmos de clusterização de grafos e a segunda como uma estratificação dos indivíduos a partir de múltiplas modalidades de dados. Nós detalhamos os objetivos específicos na seção 1.1 deste capítulo. No Capítulo 2 , nós descrevemos todo o trabalho desenvolvido para a proposta dos dois algoritmos de clusterização em grafos, incluindo uma introdução ao tema (Seção 2.1), métodos (Seção 2.2), resultados e discussão (Seção 2.3) e conclusão (Seção 2.4). No Capítulo 3 nós descrevemos todas as etapas da estratificação do espectro autista com base em associação cérebrocomportamental utilizando SPLS, incluindo uma introdução ao tema (Seção 3.1), métodos (Seção 3.2), resultados (Seção 3.3), discussão (Seção 3.4) e conclusão (Seção 3.5). No Capítulo 4 fazemos as considerações finais deste trabalho e no Capítulo 5 citamos as produções textuais relacionadas aos resultados obtidos neste trabalho.

\subsection{Objetivos}

Os objetivos específicos deste trabalho são divididos em duas partes: os objetivos referentes à clusterização de grafos e os objetivos referentes à estratificação do espectro autista.

Os objetivos específicos da clusterização de grafos são:

1. Agrupar $N$ grafos em $K$ clusters de forma que os grafos gerados pelo mesmo modelo de grafo aleatório pertençam ao mesmo cluster. 
1.1 OBJETIVOS

2. Avaliar o desempenho dos algoritmos utilizando dados simulados.

3. Estimar o ponto em que um macaco perde a consciência em uma tarefa de anestesia utilizando o algoritmo k-means para grafos.

4. Separar compostos químicos de dois bancos de dados diferentes utilizando o algoritmo gCEM.

Os objetivos específicos relacionados à estratificação dos indivíduos são:

1. Encontrar uma associação cérebro-comportamental em uma amostra de indivíduos com DT e TEA.

2. Elucidar as redes cerebrais que estão associadas a comportamentos característicos do TEA. 



\section{Capítulo 2}

\section{Clusterização de indivíduos com base em conectividades funcionais cerebrais}

\subsection{Clusterização de grafos}

A clusterização é uma das técnicas mais amplamente utilizadas para análise exploratória de dados. Em ciências naturais, geralmente é o primeiro passo para reduzir o problema principal a problemas mais simples. Quando o conjunto de dados é composto de grafos, não é diferente. As abordagens mais comuns são a detecção de comunidade (BRANDES et al., 2008), algoritmos de clusterização espectral (Spielman e Teng, 2007; LuxbURG, 2007) e propagação de rótulo (X. Zhu e Ghahramani, 2002; Fujiwara e Irie, 2014; Ugander e BACKSTROM, 2013). No entanto, todos esses métodos estão focados em clusterizar os vértices do grafo (Figura 2.1 A), ou seja, eles consideram os vértices altamente interconectados como pertencentes ao mesmo cluster. Neste trabalho, não estamos interessados em clusterizar os vértices do grafo em subgrafos, mas sim em clusterizar uma coleção de grafos em subgrupos de grafos com estruturas semelhantes (Figura 2.1 B).

O principal problema de agrupar grafos empíricos é a aleatoriedade intrínseca. Por exemplo, duas redes cerebrais ou metabólicas funcionais são diferentes, mesmo pertencendo ao mesmo grupo (grupo saudável). Nesse caso, consideraríamos dois grafos "iguais" como pertencentes a clusters diferentes apenas devido à sua aleatoriedade. Assim, a comparação direta de suas estruturas parece ser limitada. Nesse contexto, uma propriedade interessante do grafo é a densidade espectral (Wilson e P. ZHU, 2008; Wills e Meyer, 2020). Os grafos gerados por diferentes modelos de grafos aleatórios apresentam diferentes densidades espectrais. Outra vantagem desta representação é sua independência de correspondência de vértices, o que ajuda a identificar redes que apresentam padrões de conectividade semelhantes (Demirci et al., 2008; Wilson e P. Zhu, 2008).

Com base na densidade espectral, propomos uma medida de distância e um algoritmo inspirado em k-means (MACQueEN et al., 1967; Lloyd, 1982) para agrupar grafos. No entanto, os grafos gerados pelo mesmo modelo de grafo aleatório e parâmetros, ou seja, 

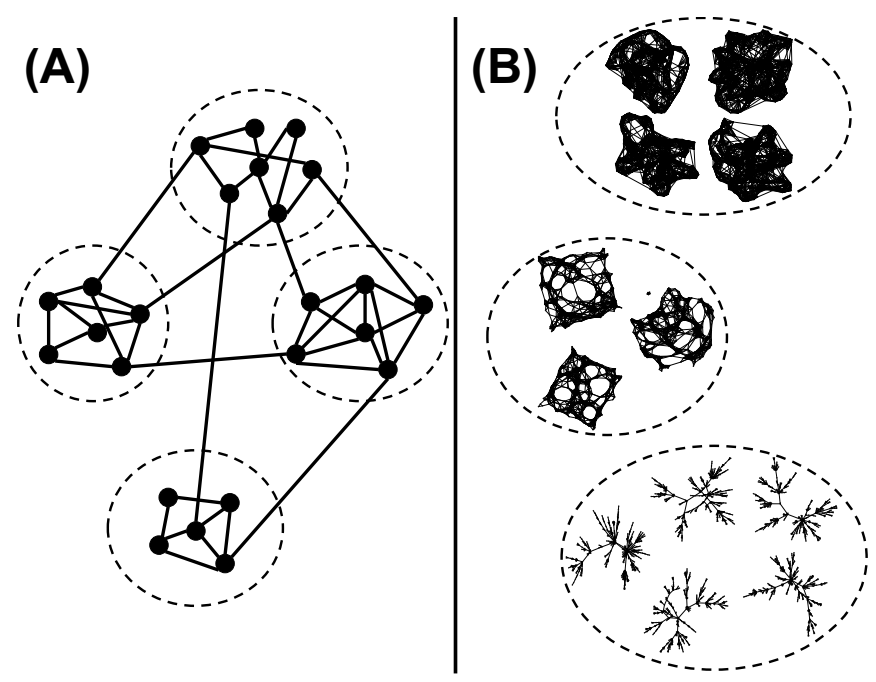

Figura 2.1: Esquemático da clusterização de grafos. (A) Agrupando os vértices de um grafo em subgrafos. Vértices altamente interconectados formam um cluster. (B) Os grafos gerados pelo mesmo modelo de grafo e o mesmo conjunto de parâmetros pertencem ao mesmo cluster, enquanto os grafos gerados por diferentes modelos, ou os mesmos modelos com um conjunto diferente de parâmetros, pertencem a diferentes clusters.

grafos que devem ser agrupados, podem apresentar tamanhos diferentes. Neste caso, os suportes das densidades espectrais são diferentes, e não é possível comparar os grafos usando as medidas de divergência entre os espectros.

Para comparar grafos de tamanhos diferentes, usaremos uma abordagem baseada em modelo. A ideia é baseada na abordagem de clusterização de mistura gaussiana (CELEUX e Govaert, 1992; Celeux e Govaert, 1995), onde representamos cada cluster por uma distribuição gaussiana. Aqui, assumimos que dois ou mais modelos de grafos geram as redes. Assim, atribuímos redes geradas pelo mesmo modelo ao mesmo cluster e redes geradas por modelos diferentes a clusters diferentes.

Para avaliar o desempenho de nossos algoritmos de clusterização de grafos, projetamos três simulações com cenários de tamanhos variados de grafos e modelos de grafos aleatórios. Finalmente, ilustramos as possíveis aplicações de nossa proposta em dois conjuntos de dados biológicos.

\subsection{Métodos}

\subsubsection{Densidade espectral de grafos}

Seja $G=(V, E)$ um grafo composto de um conjunto $V$ de $|V|$ vértices e um conjunto $E$ de $|E|$ arestas onde cada aresta em $E$ conecta dois vértices em $V$. Qualquer grafo não direcionado $G$ pode ser representado por sua matriz de adjacência $\mathbf{A}|V| \times|V|$ onde $\mathbf{A}_{i j}=$ $\mathbf{A}_{j i}=1(i, j=1, \ldots,|V|)$ se os vértices $i$ e $j$ estiverem conectados, e 0 caso contrário.

O espectro de $G$ é o conjunto de autovalores $\left(\lambda_{1} \geq \lambda_{2} \geq \ldots \geq \lambda_{|V|}\right)$ da matriz de adjacência A. Observe que, como $G$ não é direcionado, A é simétrico e, conseqüentemente, 
$\left(\lambda_{1}, \lambda_{2}, \ldots, \lambda_{|V|}\right)$ são reais. Seja $\lambda$ o vetor composto por todos os autovalores de um dado grafo $G$ e $\delta$ seja a função delta de Dirac. Então, a distribuição espectral de um grafo aleatório $G$ é definida como

$$
\rho_{G}(\lambda)=\frac{1}{|V|} \sum_{j=1}^{|V|} \delta\left(\lambda-\lambda_{j}\right) .
$$

Para estimar a densidade espectral, usamos uma regressão de kernel Gaussiano com o estimador de Nadaraya-Watson. Definimos a largura de banda do kernel gaussiano por $\left(\lambda_{1}-\lambda_{|V|}\right) /$ número de bins, e o número de bins usando o critério de Sturges. Finalmente, normalizamos a área abaixo da curva para um. Importante destacar que a largura do bin pode afetar os resultdos, caso seja utilizado um critério diferente para determinar este valor.

\section{Divergência de Kullback-Leibler}

Como mencionado anteriormente, duas densidades espectrais diferentes implicam que diferentes modelos de grafos aleatórios ou um conjunto diferente de parâmetros geraram os grafos. Assim, podemos usar a divergência de densidade espectral como uma medida de dissimilaridade entre grafos. Aqui usamos a divergência de Kullback-Leibler, doravante chamada de divergência KL. Seja $\rho_{G_{1}}$ a densidade espectral de um dado grafo e $\rho_{G_{2}}$ a densidade espectral de referência. Em seguida, definimos a divergência KL como

$$
K L\left(\rho_{G_{1}} \mid \rho_{G_{2}}\right)=\int_{-\infty}^{+\infty} \rho_{G_{1}}(\lambda) \log \frac{\rho_{G_{1}}(\lambda)}{\rho_{G_{2}}(\lambda)} d \lambda,
$$

se o suporte de $\rho_{G_{1}}$ contém o suporte de $\rho_{G_{2}}$ e $K L\left(\rho_{G_{1}} \mid \rho_{G_{2}}\right)=\infty$ caso contrário (TAKAHASHI et al., 2012; Siqueira SANTOs et al., 2016). Observe que a divergência KL não é uma medida simétrica, ou seja, $K L\left(\rho_{G_{1}} \mid \rho_{G_{2}}\right) \neq K L\left(\rho_{G_{2}} \mid \rho_{G_{1}}\right)$. Assim, a divergência KL é útil nos casos em que a referência é clara.

\section{Divergência de Jensen-Shannon}

Nos casos em que desejamos comparar grafos, mas não está claro qual densidade espectral é a referência, podemos usar a divergência de Jensen-Shannon (JS). Seja $\rho_{G_{m}}=$ $\frac{1}{2}\left(\rho_{G_{1}}+\rho_{G_{2}}\right)$. Então definimos a divergência JS entre duas densidades espectrais $\rho_{G_{1}}$ e $\rho_{G_{2}}$ como

$$
J S\left(\rho_{G_{1}}, \rho_{G_{2}}\right)=\frac{1}{2} K L\left(\rho_{G_{1}} \mid \rho_{G_{m}}\right)+\frac{1}{2} K L\left(\rho_{G_{2}} \mid \rho_{G_{m}}\right) .
$$

A divergência JS é simétrica, ou seja, $J S\left(\rho_{G_{1}}, \rho_{G_{2}}\right)=J S\left(\rho_{G_{2}}, \rho_{G_{1}}\right)$, e não negativa. Além disso, a raiz quadrada da divergência JS satisfaz a desigualdade triangular. Assim, podemos usar $\sqrt{J S\left(\rho_{G_{1}}, \rho_{G_{2}}\right)}$ como medida da distância entre densidades espectrais $\rho_{G_{1}}$ e $\rho_{G_{2}}$.

Descreveremos dois algoritmos de clusterização para grafos baseados nas divergências de Kullback-Leibler e Jensen-Shannon nas próximas seções. 


\subsubsection{A estatística de silhueta}

A estatística de silhueta é uma medida de quão bem um item está clusterizado, dada a saída de um algoritmo de clusterização (Rousseeuw, 1987). Seja $G=\left\{G_{1}, \ldots, G_{N}\right\}$ os $N$ grafos que são clusterizados em $C=\left\{C_{1}, \ldots, C_{K}\right\}$ clusters por um algoritmo de clusterização. Denotamos a dissimilaridade entre os grafos $G_{i}$ e $G_{j}$ como $d\left(G_{i}, G_{j}\right)$. Seja $|C|$ o número de grafos em um cluster C. Então definimos a dissimilaridade de um grafo $G_{i}$ ao cluster $\mathrm{C}$ como a dissimilaridade média do grafo $G_{i}$ a todos os grafos em $C$ :

$$
d\left(G_{i}, C\right)=\frac{1}{|C|} \sum_{G_{c} \in C} d\left(G_{i}, G_{c}\right)
$$

Denotamos $C_{i}$ como o cluster ao qual $G_{i}$ foi designado pelo algoritmo de clusterização. Definimos a dissimilaridade do grafo $G_{i}$ ao cluster que ele pertence como $a_{i}=d\left(G_{i}, C_{i}\right)$ e a dissimilaridade do grafo $G_{i}$ ao cluster mais próximo que ele não pertence como $b_{i}=$ $\min _{C_{j} \neq C_{i}} d\left(G_{i}, C_{j}\right)$. Assim, podemos medir o quão bem cada grafo $G_{i}$ (para $i=1, \ldots, N$ ) foi clusterizado analisando a estatística de silhueta dada por:

$$
s_{i}= \begin{cases}\frac{b_{i}-a_{i}}{\max \left\{b_{i}, a_{i}\right\}}, & \text { se }\left|C_{i}\right|>1, \\ 0, & \text { se }\left|C_{i}\right|=1 .\end{cases}
$$

A estatística de silhueta $s_{i}$ assume um valor entre -1 e 1 e sua interpretação segundo Rousseeuw, 1987 é a que segue. Quando $s_{i} \approx 1, a_{i} \ll b_{i}$, isso significa que o segundo melhor cluster está mais longe do que o cluster ao grafo $G_{i}$ foi atribuído e, portanto, ele foi atribuído ao cluster mais apropriado. Se $s_{i} \approx 0$, então $a_{i} \approx b_{i}$ e neste caso não é claro se o grafo $G_{i}$ está melhor alocado no cluster atual ou no segundo melhor. Se $s_{i} \approx-1$, então $a_{i} \gg b_{i}$, o que significa que o grafo $G_{i}$ deveria ter sido atribuído ao segundo melhor cluster, pois ele está mais próximo que o cluster ao qual $G_{i}$ foi atribuído. Portanto, $s_{i}$ é uma medida de o quão boa foi a alocação feita pelo algoritmo de clusterização do grafo $G_{i}$ ao cluster $C_{i}$.

\subsubsection{K-means}

K-means é um dos algoritmos de clusterização mais populares devido à sua simplicidade. $\mathrm{O}$ algoritmo consiste em inicializar os centroides de $K$ clusters e atribuir iterativamente os itens ao cluster com o centroide mais próximo. Para adaptar k-means para nosso problema de clusterização de grafos, primeiro representaremos cada grafo por sua densidade espectral. Definimos os centroides de $K$ como a média aritmética de todas as densidades espectrais pertencentes ao cluster. A divergência JS fornece a distância entre o grafo e o centroide do cluster (consulte a seção 2.2.1). O algoritmo 1 descreve o procedimento k-means para grafos.

O k-means é sensível à condição inicial, ou seja, os resultados podem mudar dependendo de como o algoritmo atribui os $N$ grafos aos clusters no passo 2. Além disso, utiliza uma busca gulosa (passo 3), ou seja, a solução depende da ordem de atribuição dos grafos aos clusters. Consequentemente, este método pode não encontrar a solução global. Assim, 


\section{Algoritmo $1 \mathrm{~K}$-means}

Entrada: os $N$ grafos e o número de clusters $K$.

Saída: os $K$ clusters.

1: Para cada grafo $G_{i}$, computar sua densidade espectral $\rho_{G_{i}}$.

2: Seja $\mathbf{l}_{k}^{G_{i}}=1$ se o grafo $G_{i}$ pertence ao $k$-ésimo cluster, e $\mathbf{l}_{k}^{G_{i}}=0$ caso contrário $(k=$ $1, \ldots, K)$. Inicializar $\mathbf{l}_{k}^{G_{i}}$ atribuindo aleatoriamente cada grafo $G_{i}$ a um dos $K$ clusters.

3: Computar o centroide $\rho_{G_{k}}$ de cada cluster k fazendo

$$
\rho_{G_{k}}=\frac{\sum_{i=1}^{N} \mathbf{l}_{k}^{G_{i}} \rho_{G_{i}}}{\sum_{i=1}^{N} \mathbf{l}_{k}^{G_{i}}} .
$$

4: Para cada grafo $G_{i}$, computar a distância $\mathbf{d}_{k}^{G_{i}}$ entre cada grafo $G_{i}$ e o centroide do $k$-ésimo cluster fazendo

$$
\mathbf{d}_{k}^{G_{i}}=\sqrt{J S\left(\rho_{G_{i}}, \rho_{G_{k}}\right)} .
$$

5: Atualizar $\mathbf{l}_{k}^{G_{i}}$ atribuindo cada grafo $G_{i}$ ao $k$-ésimo cluster, que fornece a menor distância $\mathbf{d}_{k}^{G_{i}}$.

6: Recalcular o centroide $\rho_{G_{k}}$ para o cluster que recebeu e para o que perdeu o grafo $G_{i}$.

7: Vá para o passo 4 até que nenhum grafo seja remanejado.

recomendamos executar o algoritmo k-means várias vezes com diferentes condições iniciais e selecionar o resultado da clusterização com a maior estatística de silhueta média considerando todos os grafos (RousseEuw, 1987).

\subsubsection{Algoritmo de clusterização baseado em modelo}

Como grafos de diferentes tamanhos têm diferentes quantidades de autovalores, as densidades espectrais também são diferentes, mesmo quando o mesmo modelo de grafo aleatório os gera. Conhecemos uma normalização para a densidade espectral de alguns modelos de grafos aleatórios que permite comparar grafos de tamanhos diferentes. Por exemplo, podemos comparar as densidades espectrais de dois grafos gerados por um modelo de grafo aleatório Erdös-Rényi dividindo os autovalores por $\sqrt{|V|}$ (FARKAs et al., 2001; TRAN et al., 2013). No entanto, para a maioria dos modelos de grafos aleatórios, a normalização da densidade espectral é desconhecida. Portanto, não podemos usar o k-means para grafos com tamanhos diferentes. Para resolver este problema, propomos um método baseado em modelo. A ideia é baseada na clusterização por expectativa-maximização descrita a seguir.

\section{Algoritmo de clusterização por expectativa-maximização (CEM)}

Uma maneira de lidar com o problema tradicional de clusterização é aplicando um método baseado em modelos. Para utilizarmos o algoritmo de clusterização por expectativamaximização (CEM) (Celeux e Govaert, 1992; Celeux e Govaert, 1995), representamos cada cluster por uma distribuição paramétrica, geralmente uma distribuição Gaussiana $\mathcal{N}(\mu, \Sigma)$ onde $\mu$ é o vetor $d$-dimensional que representa a média e $\Sigma$ é a matriz $d \times d$ de covariância. Em seguida, modelamos todo o conjunto de dados por uma mistura de 
gaussianas. Queremos encontrar os parâmetros para essa mistura de gaussianas que maximiza a função de verossimilhança.

No caso em que temos dados gerados por apenas uma gaussiana, podemos encontrar os parâmetros ótimos que geram os dados observados utilizando um estimador de máxima verossimilhança (MLE). Este estimador para cada parâmetro da normal é obtido igualando a derivada parcial da função de densidade à zero. Seguindo a mesma ideia, podemos obter a função de verossimilhança $L$ para a mistura de gaussianas. Sendo $\theta=(\mu, \Sigma)$ o conjunto de parâmetros das $K$ gaussianas, $x$ os $N$ dados observados e $w$ o peso de cada uma das gaussianas, temos:

$$
L(\theta \mid x)=\prod_{i=1}^{N} \sum_{k=1}^{K} w_{k} \mathcal{N}\left(x_{i} \mid \mu_{k}, \Sigma_{k}\right) .
$$

Por simplicidade, geralmente trabalhamos com a função log da verossimilhança como segue:

$$
l(\theta \mid x)=\sum_{i=1}^{N} \log \left(\sum_{j=1}^{K} w_{j} \mathcal{N}\left(x_{i} \mid \mu_{k}, \Sigma_{k}\right)\right) .
$$

No entanto, ao igualar as derivadas parciais de 2.6 a zero e tentarmos resolver, vemos que não é possível encontrar os estimadores de máxima verossimilhança dos parâmetros na forma fechada. Por isso, utilizamos um algoitmo iterativo para resolver o problema.

A abordagem de expectativa-maximização consiste em assumir os parâmetros iniciais e ir ajustando de forma iterativa até obter uma boa estimativa. No passo de expectativa, assumimos que conhecemos os parâmetros verdadeiros (inicializados aleatoriamente ou a estimativa obtida na iteração anterior) e calculamos a probabilidade $p_{i, j}$ de cada ponto $x_{i}$ ter sido gerado por cada gaussiana $j$ da mistura. No passo de maximização, assumimos que cada ponto $x_{i}$ foi gerado pela gaussiana $j$ mais provável (abordagem hard), ou utilizamos a medida de probabilidade $p_{i, j}$ como uma ponderação (abordagem soft) e obtemos uma nova estimativa para os parâmetros. Assim, repetimos estes passos de forma iterativa, sempre melhorando a estimativa dos parâmetros até a convergência.

Descrevemos o algoritmo EM para mistura de gaussianas como segue:

\section{Adaptação do CEM para grafos (gCEM)}

Para adaptarmos o método descrito na seção 2.2.4 para grafos, primeiro precisamos de um estimador de parâmetros para grafos. Descrevemos o método utilizado a seguir.

Supondo que um determinado modelo de grafo aleatório gerou o grafo, podemos usar a divergência KL para construir um estimador de parâmetro. Seja $M$ um modelo de grafo aleatório, $\rho_{G}$ a densidade espectral do grafo $G$ e $\left\{\rho_{M}(\theta)\right\}$ uma família paramétrica de densidades espectrais indexadas por um vetor real $\theta$. Suponha que exista um valor $\theta^{*}$ do parâmetro $\theta$ que minimiza $K L\left(\rho_{G} \mid \rho_{M}(\theta)\right)$. Um estimador $\hat{\theta}$ de $\theta^{*}$ é dado por (TAKAHASHI et al., 2012; SiQUeIRA SANTOS et al., 2016) 
Algoritmo 2 Clusterização por expectativa-maximização - CEM

Entrada: o vetor $\mathbf{x}$ de $\mathrm{N}$ observações, o número de clusters $K$.

Saída: os $K$ clusters, e o conjunto de parâmetros estimados para cada cluster.

1: Inicialize aleatoriamente os parâmetros dos modelos $\left(w_{k}, \mu_{k}\right.$ e $\Sigma_{k}$, para $\left.k=1,2, \ldots, K\right)$.

2: Expectativa: Compute a probabilidade de ocorrer $x_{i}$, para $i=1, \ldots, N$ dado que os parâmetros são $\mu_{j}, \Sigma_{j}$, para $j=1, \ldots, K$.

$$
p_{i, j}=\frac{w_{j} \mathcal{N}\left(x_{i} \mid \mu_{j}, \Sigma_{j}\right)}{\sum_{k=1}^{K} w_{k} \mathcal{N}\left(x_{i} \mid \mu_{k}, \Sigma_{k}\right)}
$$

3: Maximização: atualize os parâmetros da seguinte forma:

$$
\hat{w}_{j}=\frac{\sum_{i=1}^{N} p_{i, j}}{N}, \hat{\mu}_{j}=\frac{\sum_{i=1}^{N} p_{i, j} x_{i}}{\sum_{i=1}^{N} p_{i, j}}, \hat{\Sigma}_{j}=\frac{\sum_{i=1}^{N} p_{i, j}\left(x_{i}-\mu_{j}\right)\left(x_{i}-\mu_{j}\right)}{\sum_{i=1}^{N} p_{i, j}} .
$$

4: Repetir as etapas 2 e 3 até a convergência.

$$
\hat{\theta}=\underset{\theta}{\arg \min } K L\left(\rho_{G} \mid \rho_{M}(\theta)\right)
$$

Exceto para alguns modelos (por exemplo, modelo de grafo aleatório Erdös-Rényi), a densidade espectral teórica é desconhecida. Assim, estimamos $\rho_{M}(\theta)$ usando uma abordagem de Monte Carlo. O estimador de parâmetro baseado na divergência KL é implementado na função graph.param.estimator do pacote R statGraph (https: //CRAN.R-project.org/package=statGraph).

Para fazermos uma clusterização por expectativa-maximização para grafos, a ideia geral é assumir que $K$ modelos de grafos aleatórios geram os $N$ grafos. Neste caso, o modelo de grafos aleatórios faz o papel da distribuição Gaussiana no algoritmo original e este modelo dará a distribuição dos grafos sem assumir normalidade. Começamos o algoritmo realizando a estimação de parâmetros para cada grafo utilizando o estimador 2.8 e inicializamos os parâmetros de cada cluster. Na etapa de expectativa, usamos a divergência KL como uma medida de dissimilaridade entre a densidade espectral do grafo e a densidade espectral do modelo de cada cluster. Calculamos a probabilidade condicional de um grafo pertencer a um cluster como o inverso da divergência KL normalizada entre 0 e 1. Atribuímos o grafo ao cluster com a maior probabilidade condicional (abordagem hard). $\mathrm{Na}$ etapa de maximização, re-estimamos o parâmetro do cluster como uma média dos parâmetros estimados para os grafos daquele cluster. Repetimos as etapas de expectativa e maximização até a convergência. As saídas desse algoritmo são os clusters dos grafos e os parâmetros estimados para os modelos de cada cluster. $\mathrm{O}$ algoritmo 3 descreve a abordagem de clusterização de grafos por expectativa-maximização (gCEM).

Observe que esse algoritmo também pode assumir que diferentes modelos de grafos aleatórios geram cada cluster. No entanto, neste caso, propomos executar uma abordagem de seleção de modelos (TAKAHAshi et al., 2012) para cada grafo. 
Algoritmo 3 Clusterização de grafos por expectativa-maximização - gCEM

Entrada: os $N$ grafos, o número de clusters $K$, e o modelo de grafos aleatórios $M$.

Saída: os $K$ clusters, e o conjunto de parâmetros estimados para cada cluster.

1: Para cada grafo $G_{i}$, estime o parâmetro $\theta_{G_{i}}$, assumindo que o grafo $G_{i}$ veio do modelo $M$, usando a equação 2.8 .

2: Inicialize os parâmetros $\theta_{k}$ dos $K$ clusters, para $k=1, \ldots, K$

3: Expectativa: Compute a "probabilidade condicional" $\mathbf{t}_{k}^{G_{i}}$ do grafo $G_{i}$ vir do modelo de grafos aleatórios $M$ com parâmetros $\theta_{k}$ fazendo

$$
\mathbf{t}_{k}^{G_{i}}=\frac{1 / K L\left(\rho_{G_{i}} \mid \rho_{M}\left(\theta_{k}\right)\right)}{\sum_{i=1}^{N} 1 / K L\left(\rho_{G_{i}} \mid \rho_{M}\left(\theta_{k}\right)\right)} .
$$

4: Clusterização: Seja $\mathbf{l}_{k}^{G_{i}}=1$ se o grafo $G_{i}$ pertence ao $k$-ésimo cluster, e $\mathbf{l}_{k}^{G_{i}}=0$ caso contrário. Atualize $\mathbf{l}_{k}^{G_{i}}$ atribuindo cada grafo $G_{i}$ ao $k$-ésimo cluster, que fornece o máximo $\mathbf{t}_{k}^{G_{i}}$.

5: Maximização: Compute a nova estimativa dos parâmetros

$$
\hat{\theta}_{k}=\frac{\sum_{i=1}^{N} \mathbf{l}_{k}^{G_{i}} \theta_{G_{i}}}{\sum_{i=1}^{N} \mathbf{l}_{k}^{G_{i}}} .
$$

6: Vá para o passo 3 até a convergência de $\mathbf{t}_{k}^{G_{i}} \times K L\left(\rho_{G_{i}} \mid \rho_{M}\left(\theta_{k}\right)\right)$.

\subsubsection{Modelos de grafos aleatórios}

Usamos modelos de grafos aleatórios principalmente para estudar as propriedades estruturais de redes do mundo real. Aqui, assumimos que todos os grafos não têm rótulos, são não direcionados e não têm loops. Nas seções a seguir, descrevemos resumidamente cinco modelos de grafos aleatórios: Erdös-Rényi (ERDÖs e RÉNYI, 1959), geométrico (PENRose, 1999), k-regular (BollobÁs e BÉla, 2001), Watts-Strogatz (WATts e StrogAtz, 1998), e ligação preferencial (BARABÁsi e Albert, 1999).

\section{Modelo de grafos aleatórios de Erdös-Rényi}

O modelo de grafos aleatórios de Erdös-Rényi (ERDös e RÉNYI, 1959) consiste em gerar um grafo de $|V|$ vértices e conectar cada par de vértices por uma aresta com probabilidade $p$. Uma versão alternativa é sortear uniformemente $|E|$ arestas entre todas as arestas possíveis no grafo.

\section{Modelo de grafos aleatórios geométrico}

O modelo de grafos aleatórios geométrico (PenRose, 1999) gera um grafo inicialmente dispondo uniformemente $|V|$ vértices no espaço $R^{d}$. Então, ele conecta dois vértices por uma aresta se sua distância for menor que um raio $r$. Em todos os nossos experimentos, definimos $d=2$. 


\section{Modelo de grafos aleatórios k-regular}

O modelo de grafos aleatórios k-regular (BollobÁs e BÉLA, 2001) gera grafos onde todos os vértices $|V|$ apresentam o mesmo grau $k$. Chamamos de grau do vértice o número de arestas que incidem sobre o vértice. Este modelo gera grafos conectando vértices aleatoriamente por tentativa e erro, com o grau dos vértices sendo a única restrição.

\section{Modelo de grafos aleatórios de Watts-Strogatz}

O modelo de grafos aleatórios de Watts-Strogatz (WATts e Strogatz, 1998) gera grafos com $|V|$ vértices seguindo um algoritmo gerador como segue. Primeiro, crie uma rede em formato de anel com $|V|$ vértices conectando cada vértice com seus $k$ vizinhos mais próximos, $k / 2$ de cada lado. Então, para cada aresta $(i, j)$ (onde $i<j$ ), com probabilidade $p$ substitua a aresta por $(i, l)$, onde $l \neq i \neq j$ é aleatoriamente selecionado entre todos os vértices. Este modelo cria grafos com propriedades de " mundo pequeno" (distância média entre os vértices curta e alto coeficiente de agrupamento), que também observamos em muitas redes do mundo real. Observe que conforme o parâmetro $p \rightarrow 1$, o modelo Watts-Strogatz se aproxima de um modelo Erdös-Rényi. Sua densidade espectral também se aproxima da distribuição em semicírculo típica do modelo Erdös-Rényi (FARKAS et al., 2001).

\section{Modelo de grafos aleatórios com ligação preferencial}

Modelo de grafos aleatórios com ligação preferencial geram grafos cuja distribuição dos graus segue a lei de potência ("livre de escala") (BARABÁsi e Albert, 1999). Novos vértices na rede tendem a se conectar aos vértices com graus mais altos. Esta tendência cria vértices " $h u b$ ", ou seja, vértices com um grau muito alto. Os modelos de grafos com ligação preferencial possuem diversas implementações, das quais a mais conhecida é o modelo de Barabási-Albert (BARABÁsi e AlBert, 1999). Abaixo, descrevemos o algoritmo conforme implementado no pacote igraph do R.

Comece com $m \leq|V|$ vértices em um clique, ou seja, todos os vértices estão conectados. A cada iteração, adicione um novo vértice com $m$ conexões aos vértices adicionados anteriormente do grafo. A probabilidade de que o novo vértice escolha um determinado vértice $v_{i}$ para se conectar é proporcional ao grau do vértice $v_{i}$ e ao fator de escala $p s$ da seguinte forma: $P\left(v_{i}\right) \sim \operatorname{degree}\left(v_{i}\right)^{p s}$. Novos vértices são adicionados até que o grafo contenha $|V|$ vértices.

\subsubsection{Algoritmo de troca aleatória de Markov}

No cenário (b) da simulação 2 descrito na seção 2.2.7, desejamos perturbar gradualmente a conectividade de uma rede em formato de anel preservando número de vértices, arestas e sequência de graus da rede. Para este fim, reconectamos as arestas da rede original seguindo o algoritmo de troca aleatória de Markov (MAslov e Sneppen, 2002; LANGe et al., 2014) descrito a seguir. Selecione um par de arestas $\left(i_{1}, j_{1}\right),\left(i_{2}, j_{2}\right)$, onde $i_{1} \neq j_{1} \neq i_{2} \neq j_{2} \mathrm{e}$ tente religar as arestas para obter $\left(i_{1}, j_{2}\right),\left(i_{2}, j_{1}\right)$. Caso alguma das novas arestas já exista na rede, selecione um novo par de arestas. 


\subsubsection{Simulações}

Para avaliar o desempenho de nossos algoritmos, projetamos três simulações. Cada simulação é composta por cenários com grafos gerados por diferentes modelos de grafos aleatórios descritos na seção 2.2.5. Definimos "cluster" como o conjunto de grafos gerados pelo mesmo modelo de grafo aleatório e parâmetros. Descrevemos a configuração dos cenários de simulação da seguinte forma:

1. Grafos do mesmo tamanho. Esta simulação é composta por seis cenários como segue:

(a) três modelos de grafos aleatórios de Erdös-Rényi com parâmetros $p_{1}=0.2$, $p_{2}=0.25$, e $p_{3}=0.3$

(b) três modelos de grafos aleatórios geométricos com parâmetros $r_{1}=0.15, r_{2}=$ 0.25 , e $r_{3}=0.35$;

(c) três modelos de grafos aleatórios k-regulares com parâmetros $k_{1}=2, k_{2}=4$, e $k_{3}=6$;

(d) três modelos de grafos aleatórios de Watts-Strogatz com parâmetros $p_{1}=0.05$, $p_{2}=0.10, p_{3}=0.15$ e parâmetro $k=16$ para todos os clusters;

(e) três modelos de grafos aleatórios com ligação preferencial com parâmetros $p s_{1}=1, p s_{2}=2, p s_{3}=3$ e parâmetro $m=10$ para todos os clusters;

(f) e um modelo de grafos aleatórios geométrico com parâmetro $r=0.1$, um modelo de grafos aleatórios com ligação preferencial com parâmetros $p s=1.5$ e $m=10$, e um modelo de grafos aleatórios k-regular com parâmetro $k=2$.

Observe que existem três clusters em cada cenário. Definimos o número de grafos como 10 por cluster, ou seja, um número total de $N=30$ grafos. Para avaliar o efeito do tamanho do grafo no algoritmo de agrupamento, definimos $|V|=30,60,90,120$.

2. Grafos do mesmo tamanho e número de arestas. Esta simulação é composta por dois cenários como segue:

(a) um modelo de grafos aleatórios de Watts-Strogatz com parâmetros $k=20$ e $p$ variando de 0 a 1 , em passos de 0.1 , e um modelo de grafos aleatórios Erdös-Rényi com parâmetro $|E|=1000$. Observe que, à medida que $p$ aumenta, os grafos Watts-Strogatz se tornam mais semelhantes a um grafo Erdös-Rényi. No final, os grafos não devem ser distinguíveis.

(b) comece com um modelo de grafos aleatórios de Watts-Strogatz com parâmetros $p=0$ e $k=20$ e um modelo de grafos aleatórios k-regular com parâmetro $k=20$. Em seguida, execute o algoritmo de comutação aleatória de Markov descrito na seção 2.2.6 para religar 100,200, .., 1000 pares de arestas dos grafos de Watts-Strogatz. Observe que, conforme o número de arestas reconectadas aumenta, os grafos Watts-Strogatz se tornam mais semelhantes aos grafos k-regular. No final, os grafos não devem ser distinguíveis.

Para ambos os cenários, existem dois clusters iniciais com diferentes estruturas de conectividade. O primeiro é composto de grafos Watts-Strogatz com rede em formato de anel e o segundo pode ser composto de grafos Erdös-Rényi ou k-regulares. 
Nós gradualmente aumentamos as perturbações na conectividade dos grafos WattsStrogatz, enquanto mantemos os parâmetros do segundo cluster fixos. Este processo faz com que os grafos Watts-Strogatz se aproximem da estrutura dos grafos ErdösRényi ou k-regulares. Cada cluster é composto por 10 grafos $(N=20) \operatorname{com}|V|=100$ vértices.

3. Grafos de tamanhos diferentes. Esta simulação é composta por seis cenários, conforme descrito na simulação 1 . A diferença é que sorteamos o número de vértices uniformemente no intervalo $[30,120]$ para cada grafo. Em outras palavras, grafos pertencentes a um mesmo cluster podem apresentar tamanhos diferentes.

Executamos cada cenário de simulação 100 vezes.

\subsubsection{Redes do mundo real}

Para ilustrar a aplicabilidade de nossos algoritmos de clusterização a problemas do mundo real, descrevemos dois conjuntos de dados.

\section{Redes funcionais do cérebro}

Para avaliar a aplicabilidade do k-means em dados do mundo real, usamos dados de uma "tarefa de anestesia" (YANAGAwA et al., 2013) disponível no site do projeto neurotycho (http://neurotycho.org/). O objetivo era determinar quando a rede funcional do cérebro muda de estado entre acordado e sob anestesia. Este conjunto de dados é composto por 128 (canais) de séries temporais de eletrocorticografia (ECoG) coletadas de um macaco (Macaca fuscata) a uma taxa de amostragem de $1 \mathrm{kHz}$ e amostrada para $200 \mathrm{~Hz}$ (ver NAGASAKA et al., 2011 para mais detalhes). Todos os procedimentos do experimento envolvendo o macaco foram executados pelos pesquisadores do projeto neurotycho e nós utilizamos os dados gravados de ECoG disponíveis no site para nossas análises. Analisamos os dados de experimentos realizados em dois dias distintos com o mesmo macaco, denominado Chibi, sob o anestésico Medetomidina. Para evitar mal-entendidos, chamamos os dados dos dias 1 e 2 de Chibi 1 e Chibi 2, respectivamente. Primeiro, os dados do ECoG foram coletados por alguns minutos com o macaco acordado e sentado com os braços contidos. Aos 2581 segundos para Chibi 1 e 2955 segundos para Chibi 2, uma injeção de anestésico foi aplicada ao macaco. Definimos o ponto de perda de consciência (LOC) (4 292 segundos para Chibi 1 e 4155 segundos para Chibi 2) como o momento em que o macaco não respondeu mais ao toque em sua mão ou narina com um cotonete. Um pesquisador observou o macaco sob anestesia e testou se havia resposta a estímulos físicos. Definimos o ponto de recuperação (7 295 segundos para Chibi 1 e 6533 segundos para Chibi 2) quando o macaco respondeu aos estímulos físicos com a mesma intensidade de antes da anestesia. Dividimos a série temporal do ECoG em uma janela de um segundo para construir as redes cerebrais funcionais do macaco. Calculamos o coeficiente de correlação de Pearson entre as séries temporais de cada janela, obtendo assim uma matriz de correlação $(128 \times 128)$ por segundo. Criamos uma matriz de adjacência do grafo com base na matriz de correlação, onde correlações maiores que um corte de 0.5 foram atribuídas como um na matriz de adjacência e zero, caso contrário. Amostramos um grafo a cada 10 segundos para nossa análise, totalizando uma série temporal de 876 e 794 grafos para Chibi 1 e Chibi 2, respectivamente. 


\section{Compostos químicos}

Para verificar se o gCEM pode clusterizar grafos empíricos, nós o aplicamos em um conjunto de dados no qual os grupos são conhecidos. Combinamos dois conjuntos de dados de compostos químicos diferentes chamados MUTAG e BZR, disponíveis publicamente em http://graphkernels.cs.tu-dortmund.de. O conjunto de dados MUTAG compreende 188 grafos com um número médio de vértices de 17, $93 \pm 4,58$ (média \pm desvio padrão (sd)), um número médio de arestas de 39, $58 \pm 11,39$ e um diâmetro médio de $8,21 \pm 1,84$ arestas. O conjunto de dados BZR compreende 405 grafos com um número médio de vértices de $35,75 \pm 7,26$, um número médio de arestas de 76, $71 \pm 15$, 40 e um diâmetro médio de $11,65 \pm 2,11$ arestas. O objetivo é avaliar se o gCEM pode obter dois clusters, sendo um composto por grafos do conjunto de dados MUTAG e outro por grafos do conjunto de dados BZR.

\subsubsection{Avaliação de desempenho da clusterização}

O índice de Jaccard é uma medida comumente usada para avaliação dos resultados da clusterização e está presente em muitos pacotes para este fim (GATES e AHN, 2019). Representa uma medida de similaridade entre a clusterização esperada e a obtida (LEVANDOWSKY e Winter, 1971). Um índice de Jaccard próximo a 1 indica que a maioria dos elementos está no cluster correto (R. Tibshirani e Walther, 2005). Calculamos o índice de Jaccard usando o pacote clusteval do R.

\subsection{Resultados e discussão}

\subsubsection{Aplicações em dados simulados}

Para avaliar o desempenho dos algoritmos de clusterização propostos, começamos aplicando o algoritmo k-means nos cenários da simulação 1 descritos na seção 2.2.7. Nesta simulação, cada cenário é composto por três clusters. Os cenários de (a) a (e) são compostos por três clusters gerados pelo mesmo modelo de grafos aleatórios, mas com parâmetros diferentes. O cenário (f) é composto por três clusters representados por diferentes modelos. Para avaliar se o algoritmo tem melhor desempenho conforme o número de vértices aumenta, rodamos cada cenário com $|V|=30,60,90,120$. Assumimos que os grafos gerados pelo mesmo modelo e parâmetros pertencem ao mesmo cluster. A figura 2.2 mostra os resultados obtidos pelo k-means na simulação 1. Os índices de Jaccard obtidos apresentam correlação positiva com o número de vértices dos grafos. Em outras palavras, quanto maior for o grafo, melhor será o desempenho da clusterização do k-means. É um resultado esperado porque a densidade espectral do grafo converge para uma densidade esperada quando o número de vértices tende ao infinito (MCKAY, 1981; TrAN et al., 2013; Blackwell et al., 2007; Dumitriu, PAL et al., 2012; FARKAs et al., 2001).

Na simulação 2, estamos interessados em verificar se o k-means consegue clusterizar corretamente mesmo quando os grafos têm o mesmo número de arestas. No cenário (a), começamos com dois clusters: um composto por grafos Erdös-Rényi e outro composto 


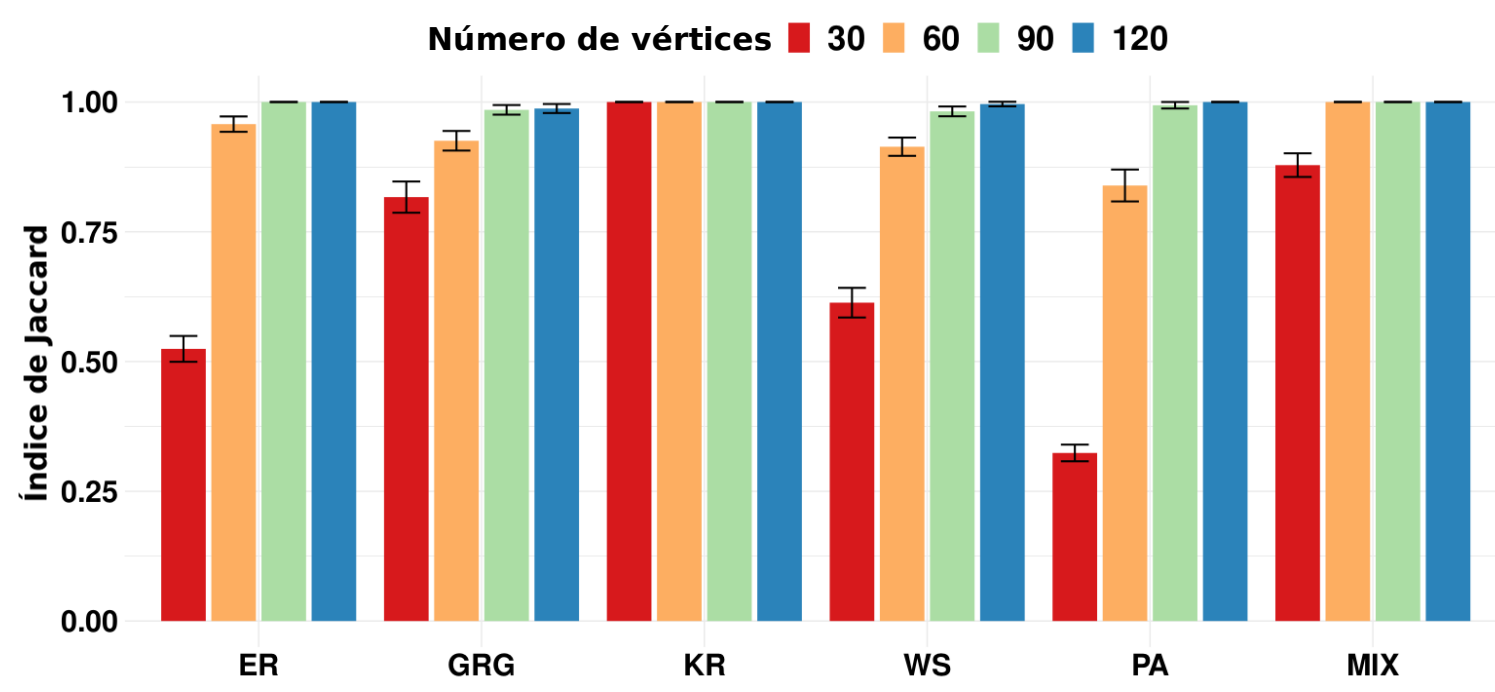

Figura 2.2: Cada grupo de barras representa um cenário da simulação 1 repetido 100 vezes, para os grafos de tamanho 30, 60, 90 e 120 vértices. Os cenários são compostos pelos seguintes modelos de grafos aleatórios: Erdös-Rényi (ER), geométrico (GRG), k-regular (KR), Watts-Strogatz (WS), ligação preferencial (preferential attachment, $P A$ ), e uma mistura de modelos de grafos aleatórios geométrico, k-regular e ligação preferencial (MIX). O eixo y representa o indice de faccard médio. As barras de erro representam intervalos de confiança de 95\%. O algoritmo k-means proposto têm melhor desempenho à medida que o tamanho do grafo aumenta.

por grafos Watts-Strogatz, todos com um número fixo de vértices $(|V|=100)$ e arestas $(|E|=1000)$. O parâmetro do Watts-Strogatz começou em $p=0$ (rede em formato de anel). Aumentamos gradualmente $p$ em passos de 0.1 para aumentar a quantidade de arestas reconectadas até que o grafo Watts-Strogatz se aproxime de um grafo Erdös-Rényi (quando $p=1$ ). A Figura 2.3 A ilustra o cenário (a). A Figura $2.3 \mathrm{~B}$ mostra o índice de Jaccard médio de 100 repetições para cada valor de $p$. Podemos ver que de $p=0$ a $p=0.3$, o k-means clusterizou corretamente os grafos. Quando $p=0.4$, ele erra alguns grafos representados pelo índice de Jaccard inferior neste ponto. De $p=0.5$ a $p=1$, o algoritmo classifica os grafos ao acaso. Portanto, este resultado mostra que o k-means consegue clusterizar corretamente grafos do mesmo tamanho e número de arestas. No entanto, quando a estrutura dos grafos passa a ser mais semelhante, o algoritmo erra a classificação, conforme esperado.

Além do número de vértices e arestas, outra característica importante do grafo é a distribuição de graus, que também pode fornecer informações suficientes para distinguir os clusters. Assim, na simulação 2, propomos o cenário (b) que mantém essas três características fixas. Começamos com dois clusters: um composto por grafos Watts-Strogatz e outro composto por grafos aleatórios k-regulares, todos com o mesmo número de vértices $(|V|=100)$, arestas $(|E|=1000)$ e grau de vértice $(d=20)$. Em cada etapa, geramos grafos Watts-Strogatz com o parâmetro $p=0$ e reconectamos as arestas usando o algoritmo de troca aleatória de Markov descrito na seção 2.2.6. Aumentamos o número de trocas em 100 a cada passo, de forma que os grafos de Watts-Strogatz se aproximam de grafos aleatórios k-regulares conforme o número de trocas aumenta. A Figura 2.3 C ilustra o design do cenário (b). A Figura $2.3 \mathrm{D}$ mostra os índices de Jaccard para cada número de trocas nos 
grafos de Watts-Strogatz. Podemos ver que de 0 a 400 trocas, o algoritmo foi capaz de clusterizar todos os grafos corretamente. Entre 500 e 600 trocas, o algoritmo errou alguns grafos, conforme representado pelos índices de Jaccard mais baixos. A partir de 700 trocas, o algoritmo dividiu os grafos em clusters ao acaso.

Grafos empíricos raramente apresentam o mesmo tamanho. Por isso, propomos o algoritmo gCEM, descrito na seção 2.2.4. Avaliamos o gCEM usando a simulação 3 descrita na seção 2.2.7. Esta simulação é composta pelos mesmos cenários usados na simulação 1. Porém, sorteamos o número de vértices dos grafos uniformemente entre 30 e 120. A Figura 2.4 mostra que o gCEM apresenta um bom desempenho em todos os cenários avaliados. Como os tamanhos do grafo variaram de 30 a 120, o número médio esperado de vértices é 75. Observe que os resultados são semelhantes aos que obtivemos quando o tamanho dos grafos está entre 60 e 90 na Figura 2.2.

\subsubsection{Aplicações em dados empíricos}

Ilustramos a aplicação do k-means e do gCEM em dois conjuntos de dados biológi$\cos$.

\section{Redes funcionais do cérebro}

No conjunto de dados da tarefa de anestesia, estamos interessados em identificar quando a rede funcional do cérebro (FBN) do macaco muda do estado de acordado para anestesiado (ou vice-versa). Na prática clínica, o momento da perda de consciência (LOC) é geralmente estabelecido pela responsividade a estímulos auditivos ou de toque (AN et al., 2018). Aqui, propomos clusterizar os FBNs do macaco em grupos de acordo com o estado atual da rede (acordado ou anestesiado) e identificar os pontos de transição de estado mental do macaco.

Como todos os FBNs apresentam o mesmo número de vértices, aplicamos o algoritmo k-means proposto. Observe que temos uma duplicata biológica, ou seja, dois conjuntos de dados coletados em dias diferentes para o mesmo macaco. Assim, realizamos a mesma análise para o primeiro e segundo dia. Para cada FBN, obtivemos um rótulo definindo a qual cluster ele pertence. Adotamos o rótulo 1 para representar o estado acordado e o rótulo 0 para representar o estado anestesiado. Para identificar o ponto de transição do estado mental, primeiro dividimos os FBNs em dois subconjuntos: um se referindo à transição de estado de acordado para anestesiado (ponto LOC) e o outro se referindo à transição de estado de anestesiado para acordado (ponto de recuperação). Para o primeiro subconjunto, incluímos os FBNs obtidos desde o início do experimento até o momento da injeção do anestésico. Incluímos a mesma quantidade de FBNs após a injeção anestésica para manter a anestesia como referência central. Para o segundo subconjunto, incluímos os FBNs obtidos a partir do momento em que o pesquisador certificou que o macaco estava acordado até o final do experimento. Incluímos a mesma quantidade de FBNs antes de o pesquisador verificar que o macaco estava acordado para manter esse ponto como referência central. Como a transição entre os estados mentais é um processo suave, esperávamos ver uma 
(A)

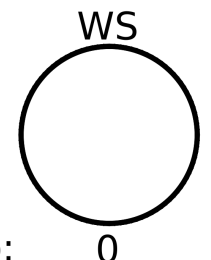

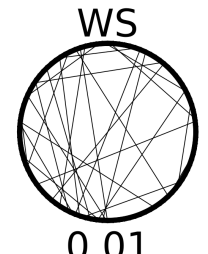

0.01

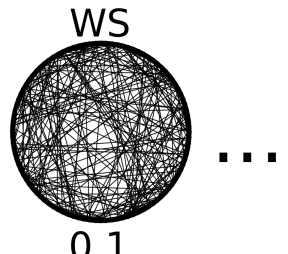

0.1
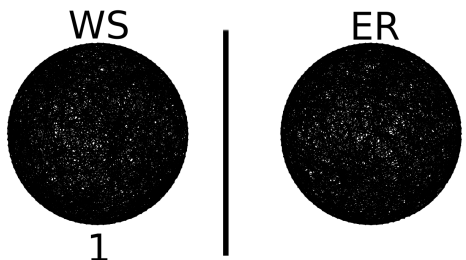

(B)

Clusterização de grafos Watts-Strogatz e Erdös-Rényi

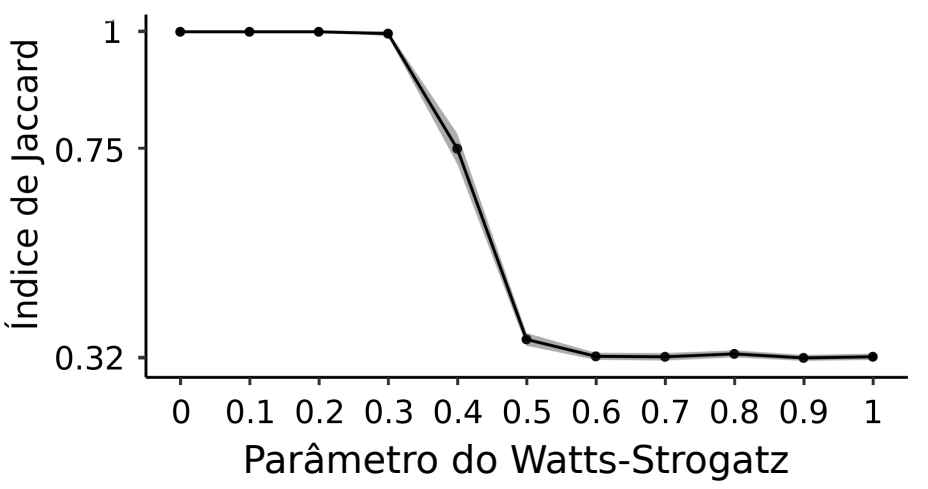

(C)
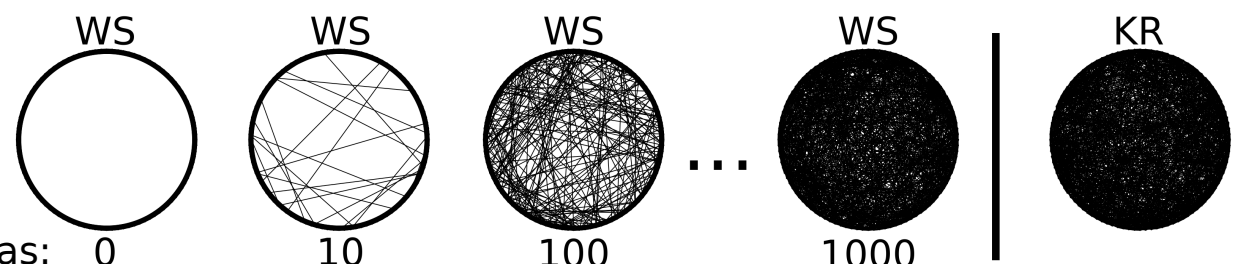

(D)

Clusterização de grafos Watts-Strogatz e k-means

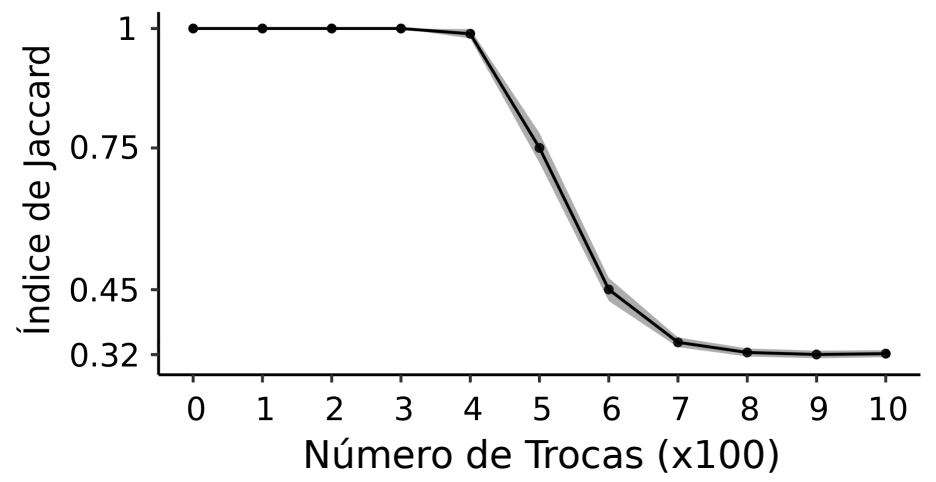

Figura 2.3: Representação gráfica e resultados da simulação 2. (A) Representação gráfica do cenário (a), ou seja, variando o parâmetro dos grafos de Watts-Strogatz (WS) até se aproximar de um grafo Erdös-Rényi (ER). (B) Resultados do cenário (a). Cada ponto representa o índice de faccard médio de 100 execuções. A área sombreada representa o intervalo de confiança de 95\%. (C) Representação gráfica do cenário (b), ou seja, reconectando as arestas dos grafos de Watts-Strogatz (WS) até se aproximar de um grafo k-regular (KR). (D) Resultados do cenário (b). Cada ponto representa o índice de faccard médio de 100 execuções. A área sombreada representa o intervalo de confiança de 95\%. Para ambos os cenários, o índice de faccard de 0.32 corresponde a $50 \%$ de precisão.

janela de tempo em que os grafos são classificados aleatoriamente até que a transição 


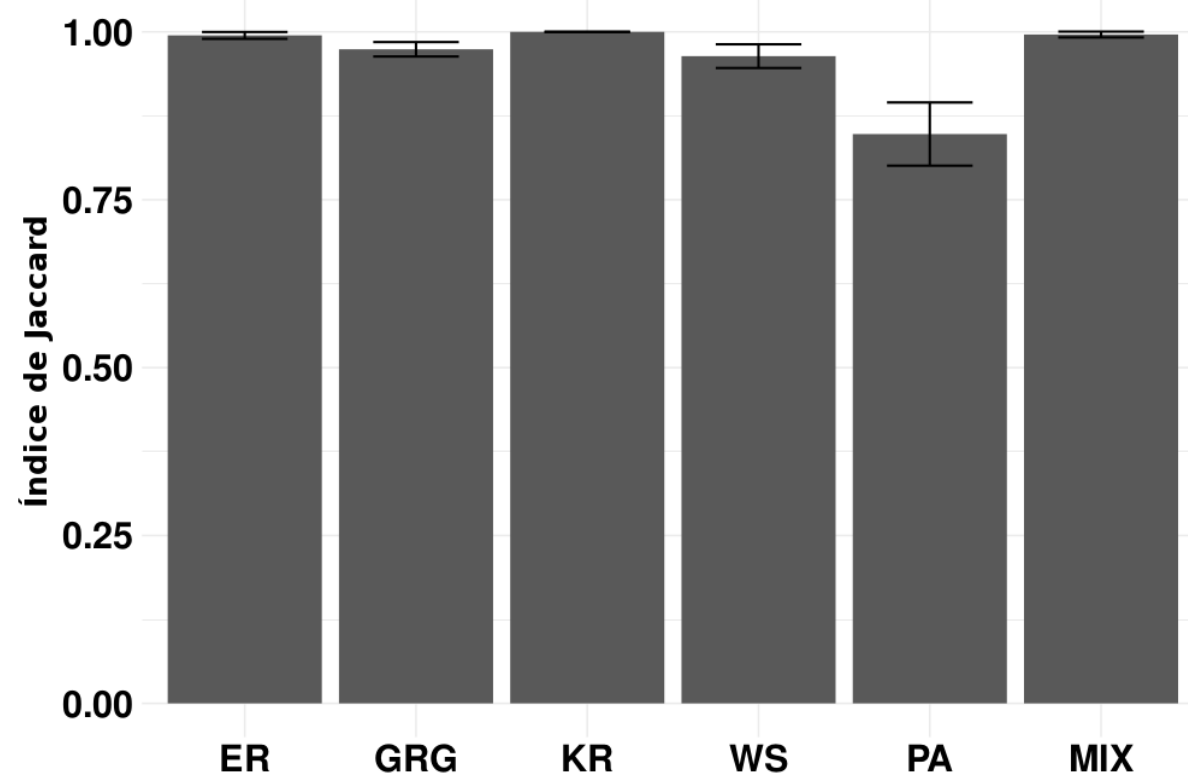

Figura 2.4: Cada barra representa um cenário de simulação 3 em 100 repetições. Sorteamos os tamanhos dos grafos uniformemente entre 30 e 120. Os cenários são compostos dos seguintes modelos de grafos aleatórios: Erdös-Rényi (ER), geométrico (GRG), k-regular (KR), Watts -Strogatz (WS), ligação preferencial (PA) e uma mistura de modelos de grafos aleatórios geométrico, $k$-regular e ligação preferencial (MIX). O eixo y representa os índices de faccard médios obtidos em 100 repetições. As barras de erro representam o intervalo de confiança de 95\%. O algoritmo gCEM consegue clusterizar corretamente, mesmo quando os tamanhos dos grafos são diferentes.

se estabilize. Assim, estimamos o ponto de tempo de transição calculando uma média móvel centralizada de 50 pontos dos rótulos de clusterização de cada subconjunto. Para o primeiro subconjunto, definimos o ponto LOC previsto para ser o primeiro ponto de tempo quando a média móvel é inferior a 0.5. Definimos o momento de recuperação previsto para o segundo subconjunto como o primeiro momento em que a média móvel é maior que 0.5 .

Os painéis da esquerda da Figura 2.5 representam o primeiro subconjunto de grafos para a transição de acordado para anestesiado. Os dois painéis à direita representam os grafos da transição de anestesiado para acordado. Os eixos x e y representam o tempo e o rótulo da clusterização, respectivamente. Cada ponto de tempo contém um FBN correspondente para o macaco representado por um círculo preto indicando seu estado mental previsto naquele momento. Nos painéis da esquerda, as linhas verticais sólidas verdes representam o momento da injeção do anestésico. As linhas verticais sólidas azuis representam o momento em que o pesquisador certificou que o macaco parou de responder aos estímulos físicos. As linhas verticais azuis tracejadas representam nosso ponto de tempo LOC previsto. Observamos que o ponto de tempo LOC estimado é após o momento da injeção do anestésico e antes do momento em que o pesquisador certificou que o macaco foi anestesiado. O ponto de tempo LOC previsto antecede o momento em que o pesquisador indicou que o macaco não respondeu a estímulos externos em 22 minutos para Chibi 1 e 14 minutos para Chibi 2. Nos painéis direitos, as linhas verticais sólidas laranja representam o momento em que o pesquisador certificou que o macaco voltou a responder aos estímulos 
físicos com a mesma intensidade antes da injeção do anestésico. As linhas tracejadas laranja representam nossa previsão do ponto de recuperação da anestesia. O ponto de tempo previsto precede o momento em que o pesquisador indica que o macaco estava acordado em 3.8 minutos para Chibi 1 e 2.5 minutos para Chibi 2. Nossos resultados estão de acordo com o esperado e estimam o ponto de mudança de estado mental do macaco antes das observações do pesquisador.

Para verificar se nossos resultados são robustos ao corte adotado na construção da matriz de adjacência (o corte foi 0.5 , conforme descrito na seção 2.2.8), reproduzimos as análises utilizando um corte de 0.45 e 0.55 . A Figura 2.6 mostra que nossos resultados e conclusões se mantém, mesmo com esta variação no corte.
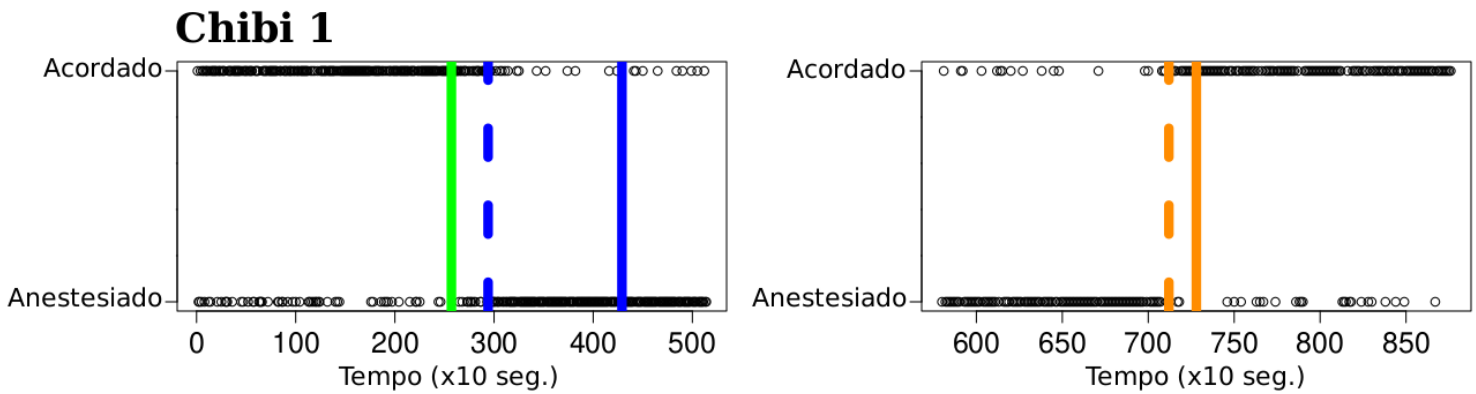

\section{Chibi 2}
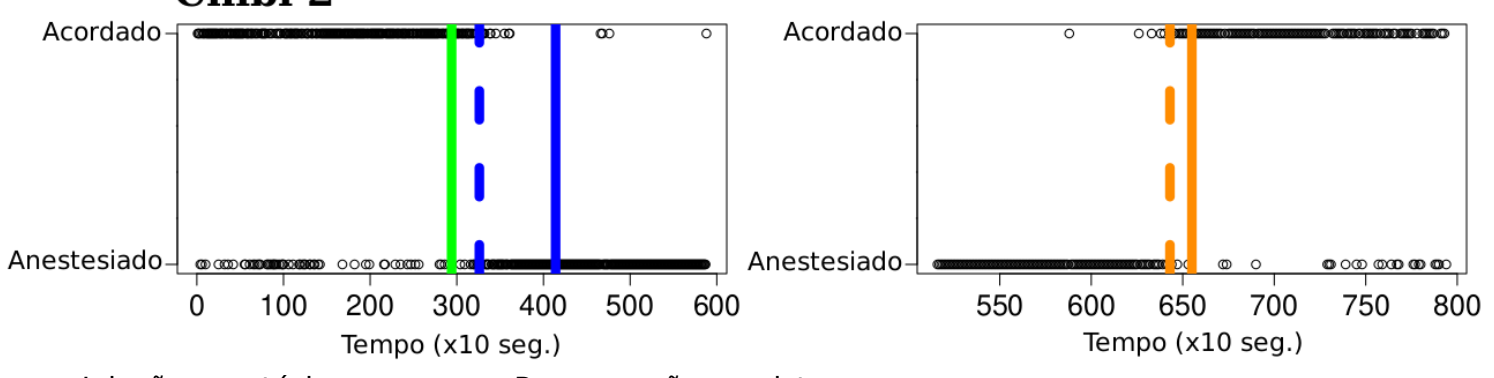

Injeção anestésica

- - Recuperação prevista

- Anestesiado previsto Anestesiado observado

Figura 2.5: Ponto de transição do estado mental. Os eixos x e y representam o tempo (em 10 segundos) e o estado mental do macaco. A linha verde representa o momento da injeção do anestésico. A linha sólida azul representa o momento em que o pesquisador certifica que o macaco parou de responder aos estímulos físicos. A linha laranja sólida representa o momento em que o pesquisador certifica que o macaco começa a responder novamente. Os círculos pretos representam o estado mental atribuído pelo $k$-means a cada FBN. Estimamos o momento em que o macaco muda seu estado mental como o ponto em que a média móvel centralizada é menor que 0.5 para os painéis esquerdos (linha tracejada azul) e maior que 0.5 para os painéis direitos (linhas tracejadas laranja). Os pontos de transição previstos ocorrem antes do pesquisador certificar o estado de transição, sugerindo uma melhor previsão do sinal de ECoG do que o teste empírico. 
(A) Chibi 1
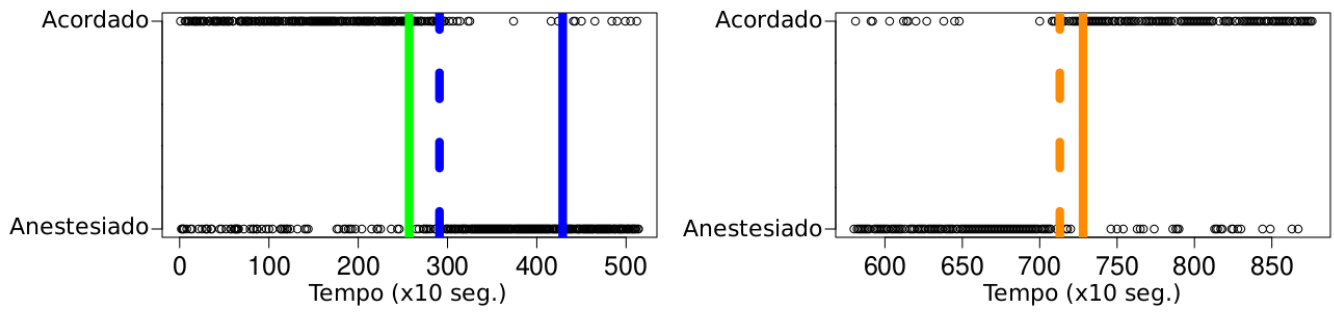

Chibi 2
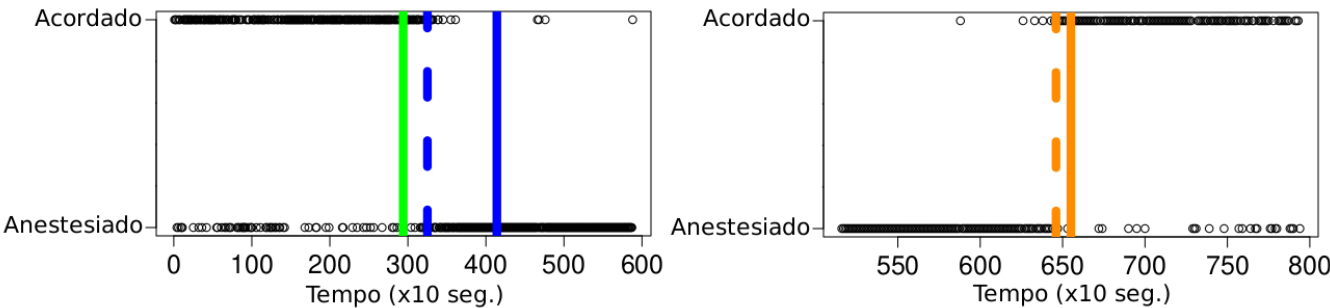

(B)
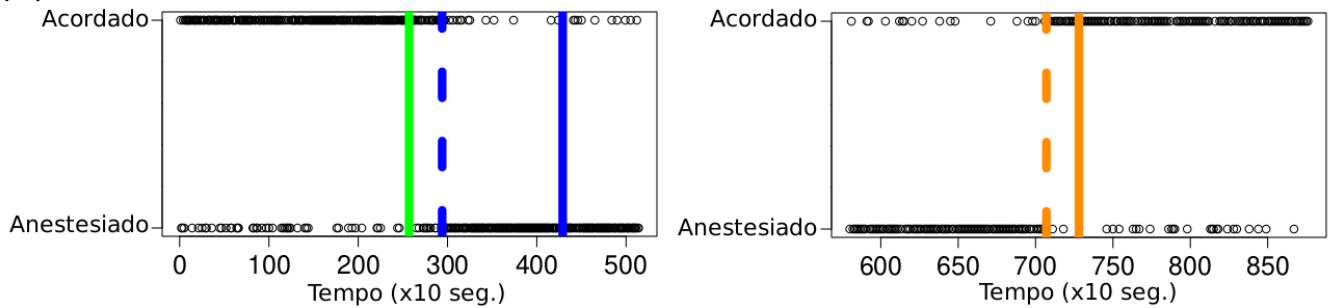

Chibi 2
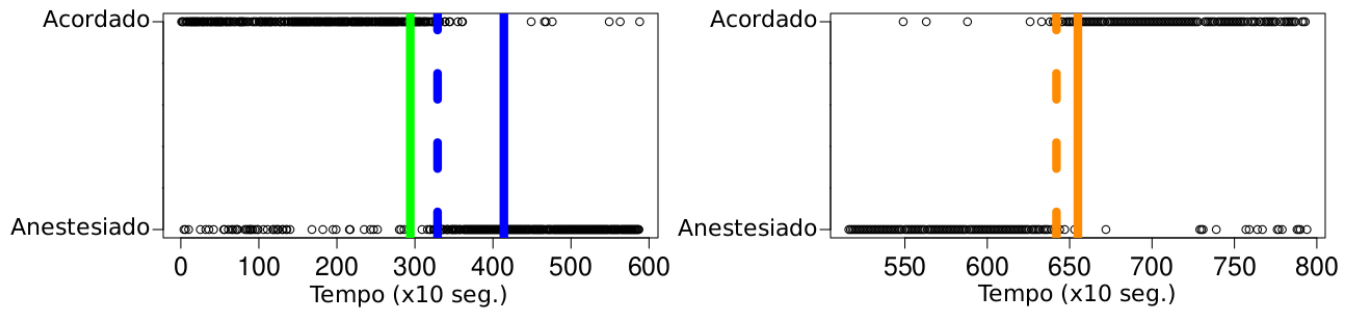

$\begin{array}{ll}\text { — Injeção anestésica } & - \text { - Recuperação prevista } \\ \text { - Anestesiado previsto } & \text { - Recuperação observada }\end{array}$

Figura 2.6: $O$ ponto de transição do estado mental usando um corte igual a 0.45 (painel A) e 0.55 (painel B) para incluir uma aresta entre dois vértices dos grafos. Os eixos $x$ e y representam o tempo (em 10 segundos) e o estado mental do macaco. A linha verde representa o momento da injeção do anestésico. A linha contínua em azul representa o momento em que o pesquisador certifica que o macaco parou de responder aos estímulos físicos, e a linha contínua em laranja representa o momento em que o pesquisador certifica que o macaco começou a responder novamente. Os círculos pretos representam o estado mental atribuído pelo k-means a cada FBN. Estimamos o momento em que o macaco muda seu estado mental como o ponto em que a média móvel centralizada é menor que 0.5 para os painéis esquerdos (linha tracejada azul) e maior que 0.5 para os painéis direitos (linhas tracejadas laranja). Os pontos de transição previstos ocorrem antes do pesquisador certificar o estado de transição, sugerindo uma melhor previsão do sinal de ECoG do que o teste empírico. Assim, a conclusão deste experimento não é afetada por uma pequena mudança $(e p s=0.05)$ no ponto de corte adotado para a geração dos grafos. 


\section{Compostos químicos}

Combinamos dois conjuntos de dados de compostos químicos diferentes chamados MUTAG e BZR. Para obter mais detalhes sobre esses conjuntos de dados, consulte a seção 2.2.8. Nosso objetivo é avaliar se o algoritmo de clusterização pode identificar de qual conjunto de dados cada composto químico veio. Como os grafos dos compostos apresentam um número diferente de vértices, usamos o algoritmo gCEM. Para definir qual modelo de grafo aleatório melhor se ajusta aos compostos químicos, aplicamos a abordagem de seleção de modelo descrita em TAKAHASHI et al., 2012. A abordagem de seleção de modelo consiste em escolher o modelo que minimiza a divergência KL entre as densidades espectrais do grafo e do modelo. A abordagem de seleção de modelos escolheu o modelo de grafos aleatórios Watts-Strogatz para $96.12 \%$ dos compostos químicos (a abordagem de seleção de modelos classificou os 23 compostos restantes como Erdös-Rényi). Este resultado não significa que o modelo Watts-Strogatz é aquele que gerou as redes moleculares, mas sim que é o modelo mais adequado entre as opções avaliadas. Para verificar se o modelo WattsStrogatz é uma boa representação para os compostos químicos, analisamos o ajuste entre as densidades espectrais dos grafos gerados pelo modelo e as densidades espectrais dos compostos químicos reais. A Figura 2.7 apresenta uma amostra ilustrativa de 20 compostos químicos e as densidades espectrais ajustadas. A zona cinza é o intervalo de confiança de 95\% para a densidade espectral ajustada. Observe que o modelo Watts-Strogatz apresenta um bom ajuste aos dados reais.

Uma vez que conhecemos os verdadeiros rótulos, ou seja, de qual conjunto de dados veio cada grafo, podemos medir o desempenho da clusterização. O algoritmo gCEM classificou corretamente 536 de 593 moléculas $(90.38 \%$ de precisão, índice de Jaccard = 0.7255). Os parâmetros estimados foram $p_{1}=0.122$ para o conjunto de dados MUTAG e $p_{2}=0.368$ para o conjunto de dados BZR. O parâmetro do modelo de grafos aleatórios Watts-Strogatz não está associado ao número de vértices nem ao número de arestas do grafo. Em vez disso, indica a aleatoriedade da disposição das arestas. Quanto menor o parâmetro, maior a probabilidade de o grafo exibir propriedades de mundo pequeno. Com base nos resultados obtidos, podemos inferir que os compostos MUTAG apresentam mais propriedades de mundo pequeno do que BZR. Em contraste, os compostos BZR apresentam uma configuração mais aleatória do que os MUTAG. 
MUTAG
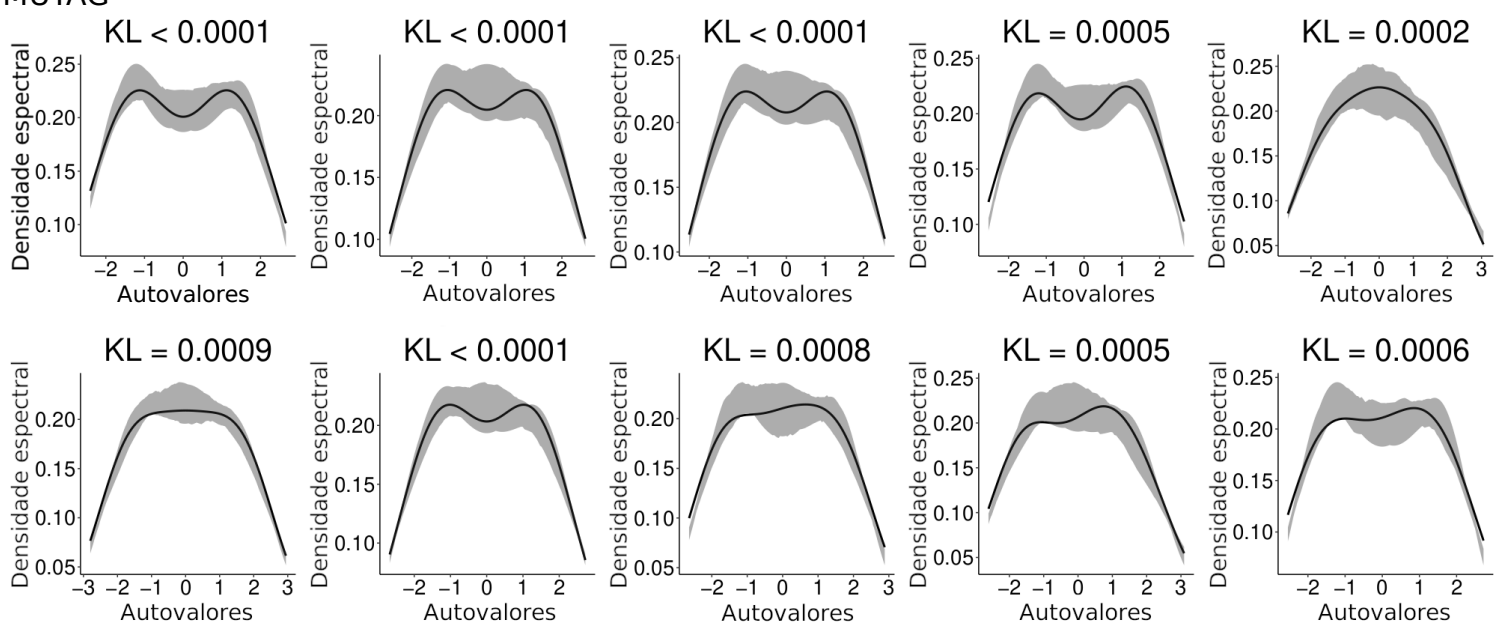

BZR
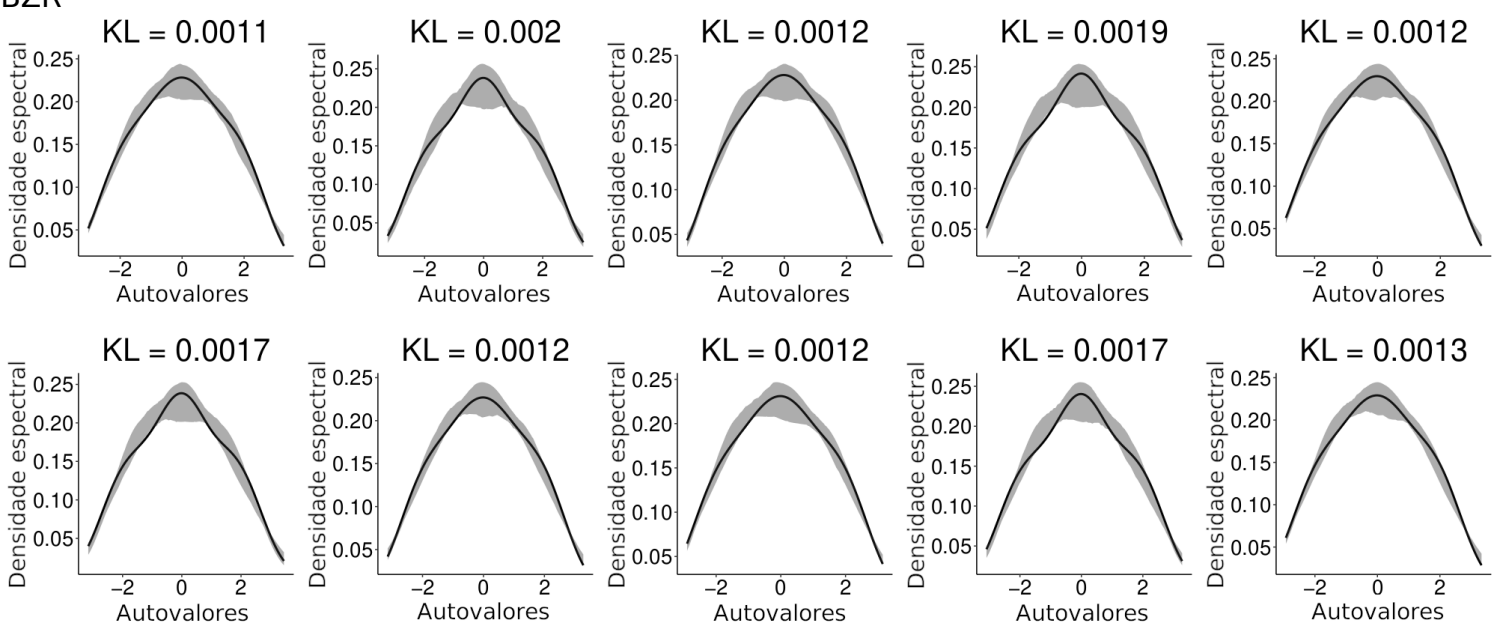

Figura 2.7: As densidades espectrais de grafos Watts-Strogatz ajustadas para 20 compostos quimicos selecionados aleatoriamente. A linha preta representa a densidade espectral do composto. A área cinza representa o intervalo de confiança de $95 \%$ para as densidades espectrais médias geradas pelo modelo Watts-Strogatz e parâmetros estimados. KL indicado representa a divergência entre o modelo Watts-Strogatz ajustado e o composto químico. Observe que a maioria das densidades espectrais dos compostos químicos (linha preta) está dentro da área cinza, indicando que o modelo Watts-Strogatz tem um bom ajuste para esses grafos.

\subsection{Conclusão}

Propusemos dois métodos de clusterização de grafos baseados na divergência de densidade espectral para clusterizar grafos semelhantes. Apresentamos primeiro o algoritmo de clusterização k-means como um método não paramétrico para clusterizar grafos. Em seguida, mostramos que ele clusteriza corretamente os grafos mesmo quando eles apresentam o mesmo número de vértices, arestas e distribuição de graus. O método k-means tem a limitação de que todos os grafos devem apresentar o mesmo número de vértices. Assim, propusemos o algoritmo gCEM baseado em modelo como alternativa. Uma vantagem do algoritmo gCEM é fornecer estimativas para os parâmetros de cada cluster, o que auxilia na interpretação das mudanças estruturais dos grafos entre os clusters. Uma limitação do algo- 
ritmo gCEM é que precisamos conhecer um modelo de grafos aleatórios que se ajuste bem aos dados empíricos. Se os grafos pertencerem a modelos diferentes, uma abordagem de seleção de modelos seria suficiente para definir os clusters. Os códigos de clusterização estão disponíveis no pacote statGraph do R (https://CRAN.R-project.org/package=statGraph), nas funções kmeans.graph e gCEM, sob licença GNU GPL. 



\section{Capítulo 3}

\section{Estratificação do espectro autista com base em associações cérebro-comportamentais}

\subsection{Associações cérebro-comportamentais em autismo}

Estima-se que o Transtorno do Espectro do Autismo (TEA) esteja presente em uma em cada 68 crianças nos Estados Unidos (Christensen, 2016) e em 1\% da população mundial (ElsabBagh et al., 2012). O diagnóstico compreende um grupo heterogêneo de transtornos do neurodesenvolvimento caracterizado principalmente por déficits na interação social, comunicação social, comportamento estereotipado ou repetitivo, interesses restritos e responsividade sensorial diferenciada (Association, 2013). Devido à falta de critérios precisos para diferenciar entre o diagnóstico de autismo, transtorno de Asperger e "Transtorno Global do Desenvolvimento Sem Outra Especificação" (PDD-NOS), a quinta edição do manual diagnóstico e estatístico de transtornos mentais (DSM-5) (Association, 2013) juntou todos esses diagnósticos sob a mesma classificação de espectro.

Embora a abordagem de estudo caso-controle pareça estar de acordo com as diretrizes diagnósticas do DSM-5 e da Classificação Estatística Internacional de Doenças e Problemas Relacionados com a Saúde décima edição (CID-10) (Association, 2013; Organization, 2004), há um número crescente de estudos sugerindo que uma abordagem estratificada seria mais apropriada para representar a natureza heterogênea do transtorno (KAPUR et al., 2012; Mottron e BzDok, 2020; Lombardo et al., 2019; TANG et al., 2020). A ideia de representar os transtornos de saúde mental como um espectro de características que variam de típicas a atípicas na população pode ser aplicada a muitos diagnósticos psiquiátricos. Essa é a essência do Research Domain Criteria (RDoC) (INSEL et al., 2010), que se propõe a combinar vários níveis de informação tais como genômica, neuroimagem e escalas psicométricas para estudar a natureza dos transtornos mentais. Em vez de um diagnóstico fixo, o RDoC visa retratar os sujeitos como pertencentes a um espectro de aspectos psicológicos e biológicos que variam de típicos a atípicos. 
A abordagem do RDoC pode ajudar a identificar biomarcadores associados a alterações comportamentais relacionadas ao diagnóstico, mas que não estão presentes em toda a amostra. Ter um marcador biológico ou fisiológico em vez de basear o diagnóstico apenas em aspectos comportamentais pode tornar o diagnóstico de transtorno mental mais preciso, ajudar a diferenciar subgrupos, fornecer diagnóstico precoce e melhorar a compreensão dos aspectos biológicos associados aos transtornos (Singh e Rose, 2009; Pratt e Hall, 2018).

Recentemente, técnicas de aprendizado de máquina aplicadas a dados psiquiátricos têm se mostrado uma grande promessa na descoberta de biomarcadores e no esclarecimento da relação entre sintomas e aspectos fisiopatológicos dos transtornos mentais (YAHATA et al., 2017; Oliveira et al., 2019; Portugal et al., 2019; Fernandes et al., 2020). No entanto, ainda não existe um biomarcador para TEA robusto com evidências suficientes para apoiar a aplicação na clínica (GoldAni et al., 2014). Seguindo a ideia do RDoC de estudar a variação de múltiplas características de típicas a atípicas, propomos estudar a associação entre dados cerebrais e comportamentais em TEA. Para isso, utilizaremos um único método estatístico multivariado, o Sparse Partial Least Squares (SPLS), que nos permite encontrar dimensões latentes que combinadas criam um espaço latente de associação multivariada entre múltiplos conjuntos de medidas coletadas dos mesmos indivíduos. Consideramos cada conjunto de medidas como uma visão de dados. Portanto, em nosso caso, temos a visão do cérebro e a visão do comportamento. Em resumo, o método SPLS identifica pesos para as variáveis que maximizam a covariância entre as visões dos dados projetadas nesses pesos. Um espaço latente, neste caso, corresponde a uma associação multivariada entre o cérebro e as variáveis comportamentais. Em outras palavras, os algoritmos identificam o espaço latente que representa a maior associação entre o cérebro e as variáveis comportamentais. Métodos multivariados se tornaram uma técnica cada vez mais popular para investigar associações entre traços de personalidade e dados de imagem do cérebro nos últimos anos (S. Smith et al., 2015; Drysdale et al., 2017; Mihalik, Ferreira, Moutoussis et al., 2020), mostrando resultados promissores para o estudo de transtornos psiquiátricos.

Especialmente para o espectro do autismo, a Imagem de Ressonância Magnética Funcional em Estado de Repouso (rs-fMRI) é uma das abordagens mais comuns para estudar a conectividade funcional do cérebro. Ele detecta flutuações espontâneas do sinal Dependente do Nível de Oxigênio no Sangue (BOLD) (BIswal et al., 1995) e a correlação nas flutuações entre as regiões é considerada um índice de conectividade funcional Fox e RAICHLE, 2007. Estudos de conectividade funcional relatam resultados mistos mostrando conectividade aumentada e diminuída no grupo TEA (MAximo et al., 2014; MAsH et al., 2018; VASA et al., 2016; King et al., 2019). Embora muitos estudos agora apoiem a hipótese de subconectividade funcional de longa distância, mais pesquisas são necessárias para investigar a hipótese de hiperconectividade de curta distância (O’REILly et al., 2017).

Do lado comportamental, selecionamos três instrumentos psicométricos: Escala de Responsividade Social (SRS) (Constantino, 2013), Lista de Verificação de Comportamento Infantil (CBCL) (ACHenbaCH, 2011) e Inventário de Avaliação Comportamental de Função Executiva (BRIEF) (GIOIA et al., 2018). O SRS mede aspectos relacionados à comunicação social, cognição social, maneirismos de autoconsciência e motivação social (Constantino et al., 2003). O CBCL mede aspectos relacionados ao comportamento infantil e à expressão afetiva, como comportamento agressivo, ansiedade, comportamento 
retraído, comportamento de internalização e externalização (HAVDAHL et al., 2016). O BRIEF mede aspectos relacionados às capacidades das funções executivas, como memória de trabalho, planejamento e execução de tarefas, organização de materiais, inibição de impulsos e regulação adequada das respostas emocionais KENWORTHY et al., 2008. Esses são aspectos-chave relacionados aos sintomas do espectro do autismo e podem fornecer uma boa representação da manifestação do transtorno no comportamento das crianças. Esses instrumentos geralmente não são aplicados no processo de diagnóstico de TEA na clínica, portanto, não esperamos que essas escalas psicométricas mapeiem o diagnóstico, mas elas representam sintomas específicos que podem ser relacionados a alterações na conexão cerebral.

Neste estudo, queremos encontrar uma associação cérebro-comportamento entre conexões funcionais do cérebro e características de comportamento, expressas de forma diferente em pessoas com desenvolvimento típico (DT) e pessoas com TEA. Esperamos que esse modo de associação nos auxilie a entender quais redes cerebrais estão relacionadas a características de comportamento potencialmente alteradas no TEA.

\subsection{Métodos}

\subsubsection{Dados}

Baixamos o mais recente conjunto de dados de ressonância magnética funcional do autismo em estado de repouso lançado pelo projeto Autism Brain Imaging Data Exchange (ABIDE) (Di MARTino et al., 2017), ou seja, ABIDE II. Este conjunto de dados é composto por 593 indivíduos com DT e 521 indivíduos com TEA (totalizando 1114 indivíduos), coletados em 19 locais. Após a exclusão de indivíduos com problemas de qualidade de imagem ou sem dados comportamentais e demográficos (critérios descritos nas seções 2.2 a 2.6), o conjunto de dados final usado neste estudo foi composto por 217 indivíduos com DT (147 homens, idade média $=10.74 \pm 2.35)$ e 177 indivíduos com TEA $(152$ homens, idade média $=$ $10.21 \pm 2.98$ ). De acordo com o projeto ABIDE (Di MARTINo et al., 2017), a coleta de dados e o compartilhamento de uma versão totalmente anonimizada dos conjuntos de dados foram aprovadas pelos respectivos comitês de revisão institucional ou comitês de ética de cada laboratório participante. Nenhuma informação protegida de saúde foi incluída. O conjunto de dados completo e mais detalhes sobre o procedimento de coleta de dados podem ser obtidos no site do projeto ABIDE (http://fcon_1000.projects.nitrc.org/indi/abide/).

\subsubsection{Pré-processamento dos dados de fMRI}

Pré-processamos os dados de fMRI seguindo o Pipeline Athena (Bellec et al., 2016), resumidos pelas seguintes etapas:

1. Exclusão dos primeiros quatro volumes.

2. Correção de temporização das fatias.

3. Correção de movimentos da cabeça. 
4. Registro da imagem dos volumes médios com a respectiva imagem anatômica do sujeito.

5. Normalização espacial para o espaço do Montreal Neurological Institute (MNI) $(4 \times 4$ × $4 \mathrm{~mm}$ de resolução).

6. Remoção dos efeitos de matéria branca, líquido cefalorraquidiano, movimento e tendência usando regressão linear múltipla.

7. Filtro temporal de passagem de banda $(0.009<\mathrm{f}<0.08 \mathrm{~Hz})$.

8. Suavização espacial usando um filtro gaussiano (FWHM $=6 \mathrm{~mm})$.

Para realizar as etapas descritas utilizamos o software Analysis of Functional NeuroImages (AFNI) (http://afni.nimh.nih.gov/afni) e a fMRIB Software Library (FSL) (http: //fsl.fMRIb.ox.ac.uk/fsl/fslwiki/). Mais detalhes sobre o pipeline Athena podem ser encontrados no site NeuroImaging Tools and Resources Collaboratory (NITRC) (www.nitrc.org/ plugins/mwiki/index.php/neurobureau:AthenaPipeline).

Os movimentos da cabeça dos indivíduos durante o exame de ressonância magnética podem levar a correlações espúrias entre as regiões. Assim, realizamos o procedimento de "scrubbing" (Power et al., 2012) para remover os quadros afetados pelo movimento da cabeça. Removemos os quadros que apresentavam Framewise Displacement (FD) maior que $0.5 \mathrm{~mm}$ e porcentagem de mudança de sinal (conforme medido pela Derivada da VARiância quadrada média sobre voxels (DVARS)) maior que 0.5\% $\triangle$ BOLD (Power et al., 2012). Não removemos os frames anteriores e seguintes ao afetado que não atendiam esses critérios. Para ser conservador, também excluímos da análise os indivíduos que apresentavam FD médio $>1 \mathrm{~mm}$ ou mais de $50 \%$ de frames com $\mathrm{FD}>0.2 \mathrm{~mm}$.

\subsubsection{Redes de conectividade funcional}

Para definir as regiões de interesse (ROIs), usamos um atlas de parcelamento funcional de 400 regiões cerebrais (CRADDOCK et al., 2012). Removemos trinta e cinco ROIs compreendendo os ventrículos ou estruturas não encefálicas usando o atlas do MNI. Assim, consideramos 316 ROIs para análise subsequente. Obtivemos a série temporal da ROI como a série temporal média dos voxels dentro da ROI. Excluímos da análise indivíduos com ROIs com sinais nulos devido às restrições de cobertura da bobina de cabeça do scanner.

Para cada sujeito, calculamos a correlação de Pearson entre as séries temporeais de todos os pares de ROIs. O valor de cada correlação de Pearson entre duas ROIs é utilizado como uma medida de conectividade funcional. Calculando a correlação entre todos os pares de ROIs, obtemos a matriz de conectividade funcional do indivíduos. Para evitar conexões repetidas, pegamos apenas a matriz triangular inferior, resultando em 49770 variáveis de conectividade funcional para cada indivíduos. O conjunto de dados formado pelas conectividades funcionais de todos os indivíduos é o que chamamos de "dados do cérebro" 


\subsubsection{Dados comportamentais e demográficos}

Precisamos de uma representação dos principais aspectos dos sintomas do espectro autista que possam fornecer uma boa caracterização da manifestação do transtorno no comportamento das crianças. Para isso, selecionamos três instrumentos psicométricos: o Social Responsiveness Scale (SRS), o Child Behavior Checklist (CBCL) e o Behavior Rating Inventory of Executive Function (BRIEF).

- Social Responsiveness Scale (SRS) (Constantino, 2013) mede aspectos relacionados à comunicação social, cognição social, autopercepção, maneirismos e motivação social. As variáveis obtidas a partir desta escala são:

- Autoconsciência: Monitorar o próprio comportamento no espaço, como por exemplo, saber se está invadindo o espaço social de outra pessoa.

- Cognição: Se refere ao processamento de informação social no contexto como um todo em vez de focar apenas em pequenos detalhes.

- Comunicação: Capacidade de interpretar comunicação social e responder a estímulos sociais de forma recíproca.

- Motivação: Iniciar interações sociais e atividades em grupo por iniciativa própria.

- Maneirismos: Comportamentos estereotipados e repetitivos, como balançar as mãos e ecolalia.

- Child Behavior Checklist (CBCL) (ACHENBACH, 2011) mede aspectos relacionados ao comportamento e à expressão afetiva da criança, como comportamento agressivo, comportamento transgressor, ansiedade, comportamento retraído, problemas de atenção, problemas sociais, queixas somáticas, comportamento de internalização e externalização. As variáveis obtidas a partir desta escala são:

- Ansiedade: Comportamentos relacionados a sintomas de ansiedade e depressão como chorar, apresentar preocupações e culpa.

- Comportamento retraído: Sintomas relacionados à depressão e comportamentos de isolamento social.

- Queixas somáticas: Sintomas físicos como dor, nausea, cansaço e tontura.

- Problemas sociais: Características de interação social problemática como se sentir rejeitado, ciumes, dependência emocional e solidão.

- Desordens do pensamento: Sintomas mentais como alucinações, insônia, automutilação, comportamentos anormais e repetitivos.

- Atenção: Sintomas relacionados à déficit de atenção.

- Atitude transgressiva: Comportamentos como usar drogas, cometer crimes, mentir, trair e não sentir culpa.

- Agressividade: Comportamentos agressivos como gritar, discutir, atacar, desobediência e teimosia. 
- Internalização: Comportamentos e sintomas relacionados a depressão e queixas somáticas.

- Externalização: Comportamentos relacionados à transgressão e agressividade.

- Behavior Rating Inventory of Executive Function (BRIEF) (GIOIA et al., 2018) mede aspectos relacionados às capacidades das funções executivas como: memória de trabalho, iniciativa para resolução de problemas, planejar e executar tarefas, organizar materiais, monitorar o próprio desempenho, inibir impulsos, desviar a atenção entre as atividades e regular as respostas emocionais de forma adequada. As variáveis obtidas a partir desta escala são:

- Controle de inibição: Controlar impulsos e parar um comportamento em um momento adequado.

- Monitoramento: Entender como o próprio comportamento afeta os outros, monitorar e avaliar o próprio desempenho durante a execução de uma tarefa.

- Mudança de foco: Transitar livremente entre atividades, mudança do foco de atenção e resolver problemas de forma flexível.

- Controle emocional: Capacidade de modular respostas emocionais de forma apropriada.

- Iniciativa: Capacidade de iniciar uma atividade de forma independente e propor ideias para solução de problemas.

- Memória de trabalho: Capacidade de reter informações ao concluir uma tarefa, ao codificar informações ou ao gerar metas e planos de forma sequencial.

- Planejamento: Capacidade de antecipar eventos futuros, definir metas, organizar e compreender os pontos centrais em apresentações escritas ou verbais.

- Organização: Capacidade de organizar os espaços de trabalho, lazer e armazenamento (por exemplo, mesas, armários, mochilas e quartos).

Também incluímos variáveis demográficas e 3 variáveis de QI, como segue:

- Idade: Idade da criança no momento da avaliação.

- Mulheres: Variável que indica se o indivíduo é mulher.

- Homens: Variável que indica se o indivíduo é homem.

- Categoria de destreza: Indica a preferência motora (destro, canhoto ou ambidestro).

- VIQ: Coeficiênte de inteligência verbal. Mede capacidades verbais e memória de trabalho.

- PIQ: Coeficiênte de inteligência de performance. Mede organização perceptual e velocidade de processamento.

- FIQ: Coeficiênte de inteligência funcional. Medida de inteligência geral, obtida a partir das duas anteiores. 
O projeto ABIDE reuniu dados previamente coletados por diversos centros, portanto, as escalas psicométricas utilizadas não são as mesmas entre os diferentes locais. Dentre todos os instrumentos psicométricos disponíveis no ABIDE, selecionamos aqueles relacionados à avaliação do comportamento e traços de personalidade que contém respostas para mais de $50 \%$ dos sujeitos. Incluímos neste estudo apenas indivíduos que possuíam pelo menos dois questionários comportamentais completos. A quantidade de dados faltantes de comportamento e demográficos é de $6,84 \%$. Para evitar a distribuição de informações confidenciais, o ABIDE fornece apenas as medidas resumidas em cada questionário e não as respostas individuais para cada item. Para manter a consistência, usamos os $t$-scores das medidas de resumo de cada questionário. No contexto de escalas psicométricas, o $t$-score é uma normalização entre 0 e 100 , com média 50 e cada 10 pontos de diferença para cima ou para baixo em relação à média representam um desvio padrão. Para ajudar na interpretabilidade, excluímos as medidas que são obtidas como uma combinação de outras medidas já incluídas na análise (por exemplo, pontuação total do SRS). Obtivemos 30 variáveis comportamentais e demográficas por sujeito, que chamamos de "dados comportamentais".

\subsubsection{Remoção de covariáveis e normalização dos dados}

Para evitar efeitos confundidores, removemos o efeito do local de coleta de dados e o FD médio fazendo uma regressão linear e pegando os resíduos. Cada variável cerebral e comportamental foi centrada na média e normalizada $\left(\mu=0, \sigma^{2}=1\right)$, e substituímos os dados ausentes pela mediana da variável correspondente em toda a amostra. Finalmente, as matrizes com dados cerebrais $\left(\mathrm{X} \in \mathbb{R}^{394 \times 49770}\right)$ e comportamentais $\left(\mathrm{Y} \in \mathbb{R}^{394 \times 30}\right)$ foram submetidas à análise SPLS.

\subsubsection{Modelagem SPLS dos dados cerebrais e comportamentais}

Realizamos uma análise multivariada utilizando o método Sparse Partial Least Squares (SPLS) para identificar padrões de conectividade do cérebro relacionados a dados comportamentais. O SPLS visa maximizar a covariância entre combinações lineares de dois ou mais conjuntos de dados, no nosso caso, os dados do cérebro e os dados comportamentais. Sendo $n$ o número de sujeitos, $p$ o número de variáveis cerebrais e $q$ o número de variáveis comportamentais, as matrizes $\mathrm{X} \in R^{n \times p}$ e $\mathrm{Y} \in R^{n \times q}$ são a entrada para o método SPLS que retorna $d=\min \{p, q\}$ modos de associação entre os conjuntos de variáveis. Cada modo de associação representa um espaço latente da relação entre cérebro e comportamento, no nosso caso. Para cada modo, o método SPLS fornece um vetor de pesos para as variáveis do cérebro $\mathbf{u} \in R^{p \times 1}$ e para as variáveis do comportamento $\mathbf{v} \in R^{q \times 1}$. Usando os pesos das variáveis, o framework calcula um score do indivíduo, referente a cada conjunto de dados, como uma combinação linear das variáveis (ou seja, a soma ponderada ou $\mathbf{P}_{X}=\mathbf{X u}$ e $\mathbf{P}_{Y}=\mathbf{Y v}$ ). Esses valores são armazenados como dois vetores, sendo um referente ao score do cérebro $\mathbf{P}_{X} \in R^{n \times 1}$ e um referente ao score do comportamento $\mathbf{P}_{Y} \in R^{n \times 1}$. Os scores cerebrais e comportamentais combinados determinam um espaço latente da relação cérebro-comportamento. Depois de encontrar cada espaço latente, removemos os efeitos da associação cérebro-comportamental encontrada tanto dos dados do cérebro como dos comportamentais (por deflação), e encontramos um novo espaço latente (modo de 
associação) utilizando o mesmo procedimento de forma iterativa. A figura 3.1 ilustra como o modelo SPLS é utilizado para identificar as dimensões latentes de associações cérebro-comportamentais.
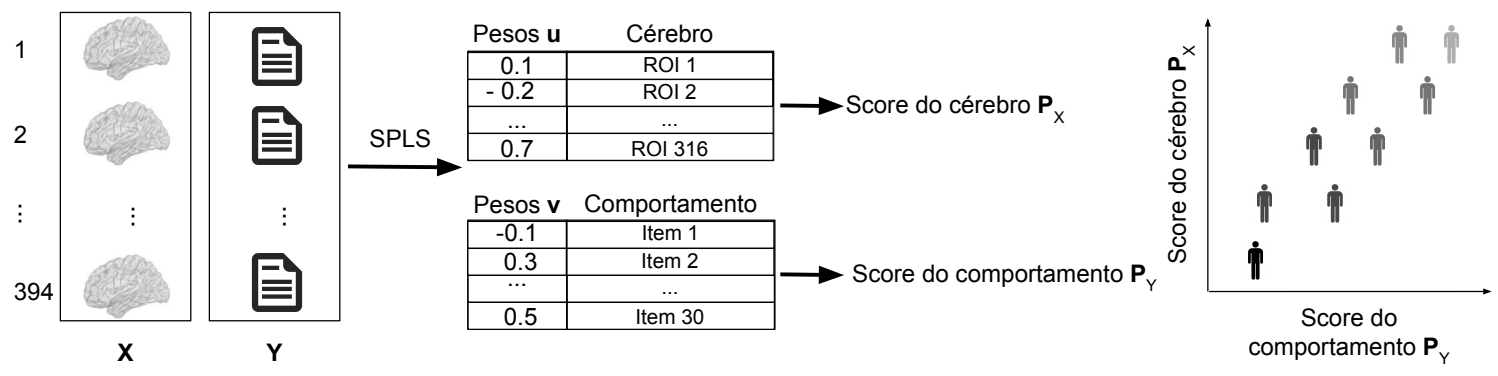

Figura 3.1: Visão geral do modelo Sparse Partial Least Squares (SPLS). O modelo SPLS busca vetores de peso $(\mathbf{u} e \mathbf{v})$ que maximizam a covariância entre as variáveis do cérebro $(\mathrm{X})$ e variáveis comportamentais $(\mathrm{Y})$. A combinação linear (soma ponderada) das variáveis cerebrais (colunas de $\mathrm{X}$ ) e comportamentais (colunas de $\mathrm{Y}$ ) com os respectivos pesos (elementos de $\mathbf{u}$ e $\mathbf{v}$ ) resulta em scores cerebrais e comportamentais $\left(\mathbf{P}_{X}\right.$ e $\left.\mathbf{P}_{Y}\right)$ para cada indivíduo. Os scores cerebrais e comportamentais podem ser combinados para criar um espaço latente que representa a expressão da associação cérebrocomportamental em toda a amostra. Figura original de MIHALIK, FERREIRA, MOUTOUSSIS et al., 2020.

\subsubsection{O método Sparse Partial Least Squares (SPLS)}

\section{Partial Least Squares (PLS)}

O PLS encontra um par de vetores de pesos $\mathbf{u}$ e $\mathbf{v}$ que maximizam a covariância entre as projeções de $\mathrm{X}$ e $\mathrm{Y}$ nos seus respectivos vetores de pesos. Seja $\|\mathbf{v}\|_{2}^{2}$ e $\|\mathbf{u}\|_{2}^{2}$ o quadrado da norma $L_{2}$ dos vetores de pesos $\mathbf{u}$ e $\mathbf{v}$, escrevemos o PLS como:

$$
\max _{\mathbf{u}, \mathbf{v}} \mathbf{u}^{\top} \mathbf{X}^{\top} \mathbf{Y v}
$$

sujeito a

$$
\|\mathbf{v}\|_{2}^{2}=1, \quad\|\mathbf{u}\|_{2}^{2}=1 \text {. }
$$

A solução da equação 3.1 pode ser obtida pela aproximação rank-1 de $\mathrm{X}^{\top} \mathrm{Y}$ utilizando Singular Value Decomposition (SVD) (LÊ CAO et al., 2008).

\section{Sparse Partial Least Squares}

Para explicarmos o método SPLS, precisamos primeiro apresentar o método de Análise de Correlação Canônica (CCA) (Hotelling, 1935). No CCA queremos encontrar vetores de pesos $\mathbf{u}$ e $\mathbf{v}$ que maximizam a correlação entre as projeções de $\mathrm{X}$ e $\mathrm{Y}$ nos vetores de pesos: 


$$
\max _{\mathbf{u}, \mathbf{v}} \mathbf{u}^{\top} \mathbf{X}^{\top} \mathbf{Y v}
$$

sujeito a

$$
\mathbf{u}^{\top} \mathbf{X}^{\top} \mathbf{X} \mathbf{u} \leq 1, \quad \mathbf{v}^{\top} \mathbf{Y}^{\top} \mathbf{Y} \mathbf{v} \leq 1 .
$$

Witten e R. J. Tibshirani, 2009 propuseram uma versão esparsa do CCA (SCCA). Adicionando uma restrição da norma $L_{1}\left(\|.\|_{1}\right)$ aos vetores de pesos utilizando parâmetros de regularização $c_{u}$ e $c_{v}$, obtemos o seguinte problema de otimização:

$$
\max _{\mathbf{u}, \mathbf{v}} \mathbf{u}^{\top} \mathbf{X}^{\top} \mathbf{Y v}
$$

sujeito a

$$
\mathbf{u}^{\top} \mathbf{X}^{\top} \mathbf{X u} \leq 1, \quad \mathbf{v}^{\top} \mathbf{Y}^{\top} \mathbf{Y v} \leq 1, \quad\|\mathbf{u}\|_{1} \leq c_{u}, \quad\|\mathbf{v}\|_{1} \leq c_{v} .
$$

Porém, assumindo que a matriz de covariância das variáveis é diagonal, os autores substituíram $\mathrm{X}^{\top} \mathrm{X}$ e $\mathrm{Y}^{\top} \mathrm{Y}$ pela matriz identidade, o que resulta no seguinte problema de otimização:

$$
\begin{aligned}
& \max _{\mathbf{u}, \mathbf{v}} \mathbf{u}^{\top} \mathbf{X}^{\top} \mathbf{Y v} \\
& \text { sujeito a } \\
& \|\mathbf{u}\|_{2}^{2} \leq 1, \quad\|\mathbf{v}\|_{2}^{2} \leq 1, \quad\|\mathbf{u}\|_{1} \leq c_{u}, \quad\|\mathbf{v}\|_{1} \leq c_{v} .
\end{aligned}
$$

Esta formulação é essencialmente uma versão esparsa do PLS (SPLS), pois o que está sendo maximizado não é mais a correlação entre as projeções, mas a covariância. A regularização da norma $L_{1}$ dos vetores de pesos garante a esparsidade desses vetores, forçando uma seleção de variáveis. Simultaneamente, a restrição da norma $L_{2}$ permite a seleção de variáveis correlacionadas (Zou e HAstie, 2005). Esta formulação do SCCA ou SPLS é bastante utilizada na literatura e é a que utilizamos neste trabalho (Rosa et al., 2015; D. A. Moser et al., 2018; Wh et al., 2018).

\section{O algoritmo do SPLS}

Witten e R. J. Tibshirani, 2009 propuseram um algoritmo iterativo para resolver a equação 3.4. Seja $S($., .) um operador de soft-thresholding definido como $S(a, \Delta)=$ $\operatorname{sgn}(a)(|a|-\Delta)_{+}$, onde $\operatorname{sgn}(a)$ é a função que retorna o sinal de $a, \Delta$ é uma constante positiva e $(x)_{+}=x$, se $x>0$ e $(x)_{+}=0$ se $x \leq 0 . \Delta_{u}$ e $\Delta_{v}$ são as constantes positivas usadas no cálculo dos vetores $\mathbf{u} \mathbf{e} \mathbf{v}$, respectivamente. Inicialmente calculamos $\mathbf{u}$ assumindo $\Delta_{u}=0$ e, caso isso resulte em $\|\mathbf{u}\|_{1}>c_{u}$, encontramos $\Delta_{u}$ usando uma busca binária tal que $\|\mathbf{u}\|_{1} \approx c_{u}$. O mesmo processo é feito para encontrar $\Delta_{v}$ tal que $\|\mathbf{v}\|_{1} \approx c_{v}$. Assim, temos o 
algoritmo:

\section{Algoritmo 4 SPLS}

Entrada: as matrizes de dados $\mathrm{X}$ e Y.

Saída: os vetores de pesos u e v.

1: Computar:

$$
\mathrm{C}=\mathrm{X}^{\top} \mathrm{Y}
$$

2: Inicializar $\mathbf{v}$ com a primeira componente do SVD de $\mathbf{C}$ para garantir $\|\mathbf{v}\|_{2}^{2}=1$

3: Atualizar u:

$$
\mathbf{u}=\frac{\mathrm{S}\left(\mathrm{Cv}, \Delta_{\mathrm{u}}\right)}{\left\|\mathrm{S}\left(\mathrm{Cv}, \Delta_{\mathrm{u}}\right)\right\|_{2}}
$$

4: Atualizar v:

$$
\mathbf{v}=\frac{\mathrm{S}\left(\mathrm{Cu}, \Delta_{\mathrm{v}}\right)}{\left\|\mathrm{S}\left(\mathrm{Cu}, \Delta_{\mathrm{v}}\right)\right\|_{2}}
$$

5: Repetir os passos 3 e 4 até a convergência de $\mathbf{u}$ e $\mathbf{v}$.

Este algoritmo é executado para encontrar um efeito de máxima covariância entre os conjuntos de variáveis. Para encontrar o próximo efeito, fazemos o processo de deflação conforme descrito a seguir na seção 3.2.8 e executamos o algoritmo novamente. A solução iterativa é interessante para o SPLS, pois assim podemos otimizar os parâmetros de regularização (esparsidade) independentemente para cada um dos efeitos encontrados.

\subsubsection{Framework de múltiplos holdouts}

Para otimizar os parâmetros de regularização do SPLS e avaliar a significância dos resultados do modelo, usamos um framework de múltiplos holdouts (multiple holdout framework) com critério conjunto de generalizabilidade e estabilidade para seleção dos parâmetros de regularização do modelo (Minalik, Ferreira, Moutoussis et al., 2020; Monteiro, Rao, Shawe-Taylor et al., 2016). Primeiro, sorteamos sem reposição indivíduos do banco de dados para formar um conjunto de otimização ( $80 \%$ dos dados originais) e um conjunto holdout (20\% dos dados originais). Cada indivíduo é representado por suas respectivas linhas das matrizes de cérebro $(\mathrm{X})$ e comportamento $(\mathrm{Y})$. O conjunto de otimização será utilizado para selecionar os parâmetros de regularização $\mathbf{c}_{u}$ e $\mathbf{c}_{v}$ do modelo SPLS. O conjunto holdout será reservado para a avaliação estatística do modelo final obtido pelo SPLS.

\section{Otimização dos parâmetros de regularização}

Para a otimização dos parâmetros utilizamos apenas o conjunto de dados de otimização separado no passo anterior. A partir deste conjunto, sorteamos sem reposição $80 \%$ dos dados para formar o conjunto de treino e $20 \%$ dos dados para formar o conjunto de validação. Repetimos este sorteio 50 vezes de forma a obter 50 divisões aleatórias (splits) dos dados em conjunto de treino e validação, conforme ilustrado na figura 3.3. A otimização dos parâmetros de regularização é feita com base em um critério conjunto de generalizabilidade e estabilidade buscando selecionar um modelo que apresente resultados consistentes para 

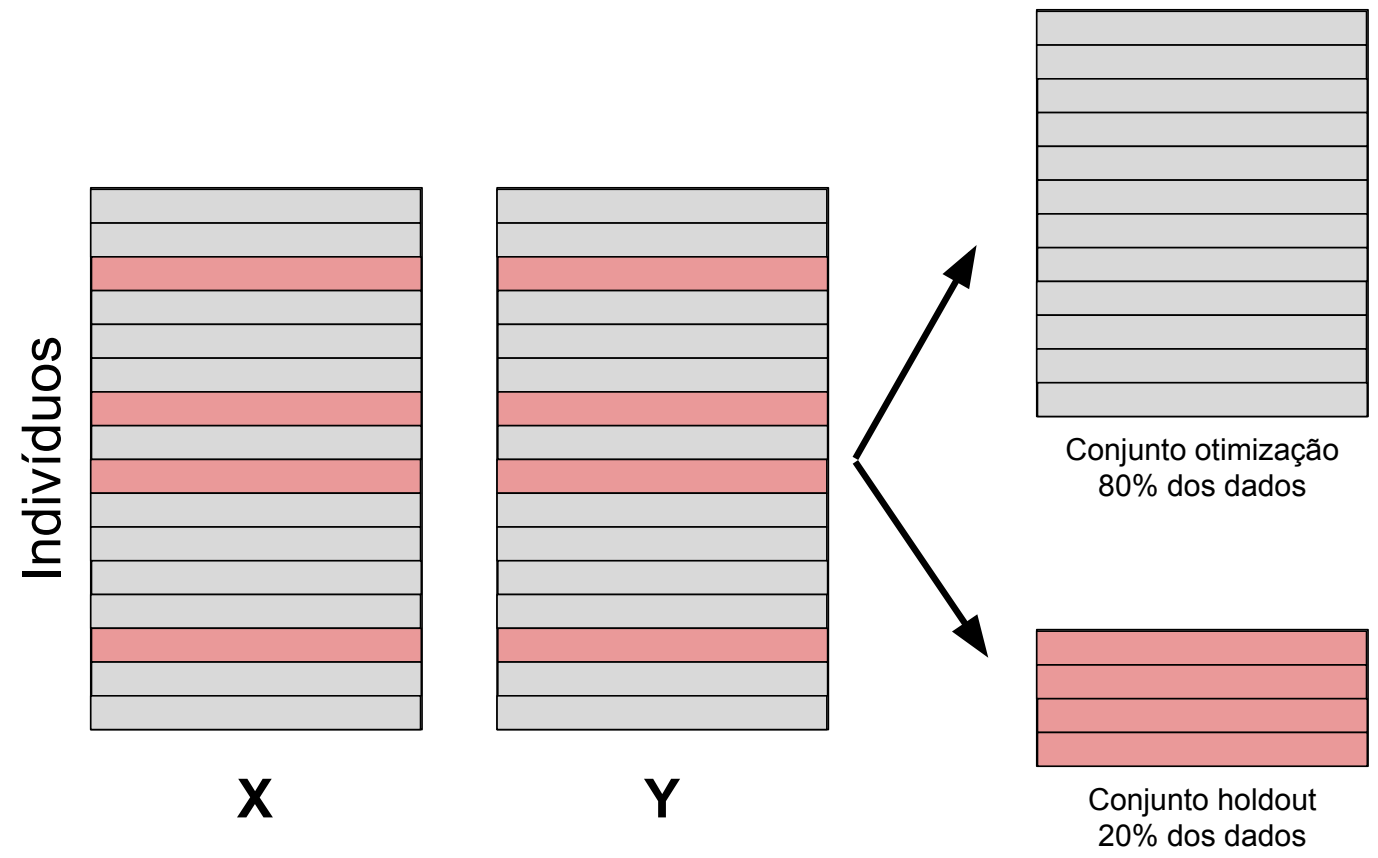

Figura 3.2: Representação da divisão dos dados em conjunto otimização (80\% dos dados) e holdout (20\% dos dados).

diferentes divisões dos dados em treino e validação. Desta forma, é esperado que o modelo consiga melhores resultados ao ser aplicado em dados nunca vistos.

Para cada combinação de parâmetros de regularização $\left(\mathbf{c}_{u}\right.$ e $\left.\mathbf{c}_{v}\right)$, ajustamos o modelo SPLS em cada um dos 50 conjuntos de treinamento. Os dados do conjunto de validação $\mathbf{X}_{\text {val }}$ e $\mathbf{Y}_{\text {val }}$ são projetados nos pesos $\mathbf{u}_{\text {treino }}$ e $\mathbf{v}_{\text {treino }}$ obtidos no conjunto de treino para obter os scores cerebrais $\mathbf{P}_{X_{\text {val }}}$ e comportamentais $\mathbf{P}_{Y_{\text {val }}}$ fazendo

$$
\mathbf{P}_{X_{\text {val }}}=\mathbf{X}_{\text {val }} \mathbf{u}_{\text {treino }}
$$

$\mathrm{e}$

$$
\mathbf{P}_{Y_{\text {val }}}=\mathbf{Y}_{\text {val }} \mathbf{v}_{\text {treino }}
$$

Para cada um dos 50 splits, computamos a correlação absoluta $\rho$ entre os scores cerebrais e comportamentais fazendo

$$
\rho=\left|\operatorname{Corr}\left(\mathbf{P}_{X_{\text {val }}}, \mathbf{P}_{Y_{\text {val }}}\right)\right| \text {. }
$$

Para cada combinação de parâmetros de regularização $\mathbf{c}_{u}$ e $\mathbf{c}_{v}$, calculamos a correlação out-of-sample absoluta média dos 50 splits fazendo

$$
\overline{\rho_{c_{u}, c_{v}}}=\frac{1}{50} \sum_{k=1}^{50} \rho_{k} .
$$

A correlação out-of-sample média determina a generalizabilidade do modelo.

O segundo critério para seleção dos parâmetros de regularização é a estabilidade. 


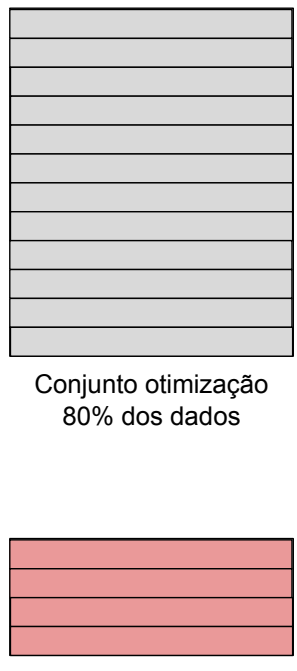

Conjunto holdout $20 \%$ dos dados

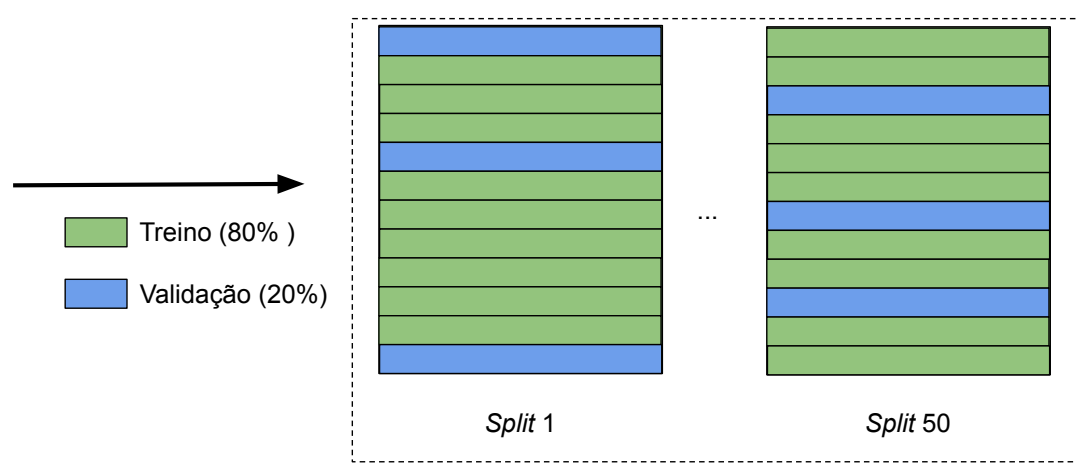

Selecionar melhores parâmetros $\left(\mathrm{c}_{\mathrm{u}}, \mathrm{c}_{\mathrm{v}}\right)$

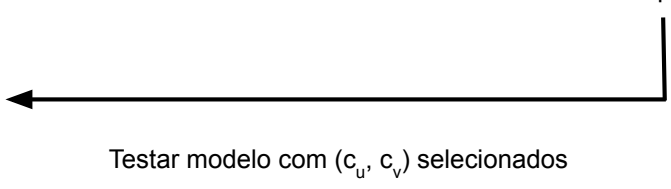

Figura 3.3: Representação das divisões dos dados para otimizar os parâmetros de regularização. Os dados originais são divididos aleatoriamente em um conjunto de otimização (80\% dos dados) e um conjunto holdout (20\% dos dados). O conjunto de otimização é usado para selecionar os parâmetros de regularização do modelo em 50 subconjuntos de treino (80\% dos dados de otimização) e validação (20\% dos dados de otimização). Ajustamos o SPLS com os parâmetros de regularização selecionados em todo o conjunto de otimização (treino + validação), e o modelo resultante é aplicado no conjunto holdout. Avaliamos o resultado estatisticamente utilizando teste de permutação.

A estabilidade é uma medida de sobreposição média das variáveis selecionadas pelos diferentes splits dos dados. Descrevemos esta medida a seguir.

Seja o vetor de pesos $\mathbf{w}_{i}$ referente ao modelo SPLS treinado no split $i$. O suporte do vetor de pesos é o conjunto $\mathbf{I}_{i}$ que contém os índices $k$ dos elementos de $\mathbf{w}_{i}$ diferentes de 0 , sendo $w_{i k}$ o k-ésimo elemento de $\mathbf{w}_{i}$, como segue:

$$
\mathbf{I}_{i}=\left\{k \mid w_{i k} \neq 0\right\} .
$$

Sendo $\left|\mathbf{I}_{i}\right|$ a quantidade de elementos do conjunto $\mathbf{I}_{i}$ e $m$ o número de elementos de $w_{i}$, chamados de esparsidade $S_{i}$ a quantidade relativa de itens não-nulos de $\mathbf{w}_{i}$, como segue:

$$
\mathrm{S}_{i}=\frac{\left|\mathbf{I}_{i}\right|}{m} .
$$

Dessa forma, a quantidade esperada de sobreposição ao acaso entre dois vetores de pesos $\mathbf{w}_{i}$ e $\mathbf{w}_{j}$ é dada por

$$
\mathbf{E}=m \times \mathrm{S}_{i} \times \mathrm{S}_{j} .
$$

Então determinamos a sobreposição corrigida de dois splits $i$ e $j$ par a par como 


$$
\mathbf{O}_{i, j}=\frac{\left|\mathbf{I}_{i} \cap \mathbf{I}_{j}\right|-E}{\max \left(\left|\mathbf{I}_{i}\right|,\left|\mathbf{I}_{j}\right|\right)}
$$

Finalmente, definimos a média da sobreposição corrigida dos pesos par a par entre todos os 50 splits como uma medida de estabilidade do modelo ajustado com os parametros $c_{u}$ e $c_{v}$, como segue:

$$
\mathbf{O}^{\prime}=\frac{1}{50 \times(50-1)} \sum_{i \leq j=1}^{50} \mathbf{O}_{i, j}
$$

Assim, considerando um espaço de busca determinado pelas medidas de generalizabilidade e estabilidade, o ponto ótimo estaria no $(1,1)$. Portanto, selecionamos como combinação ótima de parâmetros de regularização $\mathbf{c}_{u}$ e $\mathbf{c}_{v}$ a combinação cujas medidas conjuntas de generalizabilidade e estabilidade tenham menor distância euclidiana para o ponto $(1,1)$.

O modelo SPLS com os parâmetros de regularização $\mathbf{c}_{u}$ e $\mathbf{c}_{v}$ selecionados é ajustado ao conjunto de dados de otimização (treino + validação). Os dados do conjunto holdout $\left(\mathrm{X}_{\text {holdout }} \mathrm{e} \mathrm{Y}_{\text {holdout }}\right)$ são projetados nos vetores de pesos $\mathbf{u}_{\text {otim }} \mathrm{e} \mathbf{v}_{\text {otim }}$ obtidos no conjunto de otimização para obter scores de cérebro e comportamento dos indivíduos no conjunto holdout

$$
\mathbf{P}_{X_{\text {holdout }}}=\mathbf{X}_{\text {holdout }} \mathbf{u}_{\text {otim }}
$$

$\mathrm{e}$

$$
\mathbf{P}_{Y_{\text {holdout }}}=\mathbf{Y}_{\text {holdout }} \mathbf{v}_{\text {otim }} \text {. }
$$

Chamamos de correlação out-of-sample a medida de correlação entre os vetores de scores obtidos pelo SPLS no conjunto holdout. A correlação out-of-sample será usada como medida de desempenho para o modelo:

$$
\rho_{\text {model }}=\left|\operatorname{Corr}\left(P_{X_{\text {holdout }}}, P_{Y_{\text {holdout }}}\right)\right|
$$

\section{Teste de permutação}

Por fim, avaliamos a significância estatística do modelo usando um teste de permutação. Utilizando o conjunto de dados de otimização do passo anterior, começamos permutando as linhas da matriz de dados do comportamento e mantemos os dados da matriz de cérebro como o original. Assim, é esperado que não exista relação verdadeira entre os dados de cérebro e comportamento nos dados permutados. Treinamos o modelo SPLS no conjunto de dados permutados usando os parâmetros de regularização selecionados no passo de otimização. Assim obtemos novos vetores de pesos $\mathbf{u}_{\text {perm }}$ e $\mathbf{v}_{\text {perm }}$ e projetamos os dados do conjunto holdout $\left(\mathbf{X}_{\text {holdout }}\right.$ e $\left.\mathbf{Y}_{\text {holdout }}\right)$ nesses vetores para obter scores de cérebro e comportamento:

$$
\mathbf{P}_{X_{\text {perm }}}=\mathbf{X}_{\text {holdout }} \mathbf{u}_{\text {perm }}
$$




$$
\mathbf{P}_{Y_{\text {perm }}}=\mathbf{Y}_{\text {holdout }} \mathbf{v}_{\text {perm }} .
$$

A correlação out-of-sample para os dados permutados será

$$
\rho_{\text {perm }}=\left|\operatorname{Corr}\left(P_{X_{\text {perm }}}, P_{Y_{\text {perm }}}\right)\right| \text {. }
$$

Repetimos todos os passos descritos nesta sessão 1000 vezes. Assim treinamos o mesmo modelo SPLS em 1000 permutações diferentes, projetamos os dados do conjunto holdout nos vetores de pesos obtidos e obtemos 1000 correlações out-of-sample para os dados permutados. Por fim, obtivemos um p-valor para testar a significância estatística do nosso modelo SPLS obtido na etapa de otimização. Nossa hipótese nula é $H_{0}$ : "Não existe relação entre os dados do cérebro e os dados do comportamento". Se $H_{0}$ é verdadeira, a correlação out-of-sample $\rho_{\text {model }}$ obtida nos dados originais não é diferente da correlação out-of-sample $\rho_{\text {perm }}$ obtida nos dados permutados. Seja [.] os colchetes de Iverson, que recebem valor 1 se a condição $\rho_{\text {perm }} \geq \rho_{\text {model }}$ for verdadeira e 0 caso o contrário, calculamos o p-valor como segue:

$$
p-\text { valor }=\frac{\sum_{\text {perm=1 }}^{1000}\left[\rho_{\text {perm }} \geq \rho_{\text {model }}\right]}{1000} .
$$

Desta forma, se o p-valor obtido for pequeno, significa que existe uma baixa probabilidade de obter uma correlação tão grande ou maior nos dados permutados que a obtida nos dados originais. Portanto, podemos rejeitar a hipótese nula. Os passos do teste de permutação estão ilustrados na figura 3.4.

\section{Os múltiplos holdouts}

Dados de neuroimagem frequentemente apresentam um tamanho amostral pequeno, o que faz com que o conjunto holdout contenha poucos indivíduos. Isso pode gerar grande variância nos resultados a depender de como os dados foram divididos em conjunto otimização e holdout. Para fazer com que nossa análise seja mais robusta, repetimos todas as etapas descritas nas seções 3.2 .8 e 3.2 .810 vezes, obtendo 10 valores de correlação out-ofsample e 10 p-valores em 10 diferentes divisões de conjuntos de otimização e holdout. Para avaliar se existe efeito estatístico significativo nos dados que estão sendo ajustados pelo SPLS, inicialmente precisamos corrigir os p-valores obtidos para múltiplas comparações por Bonferroni. Então adotamos o conceito de hipótese omnibus, como proposto por Monteiro, Rao, Shawe-Taylor et al., 2016 para avaliação de um mesmo modelo usando múltiplas divisões dos dados. Desta forma, temos uma hipótese nula conjunta para as 10 divisões dos dados que seria $H_{\text {omnibus }}$ : "Todas as hipóteses $H_{0}$ são verdadeiras". Desta forma, basta uma das divisões dos dados obter p-valor significativo e rejeitar $H_{0}$ para rejeitarmos a hipótese nula omnibus.

Por fim, caso $H_{\text {omnibus }}$ seja rejeitada, reportamos os resultados obtidos na divisão de dados que apresentar o menor p-valor. Em caso de mais de uma divisão apresentar o mesmo p-valor, escolhemos o que tiver maior valor de correlação out-of-sample. Este resultado é o que chamamos de primeiro modo de associação cérebro-comportamental. 

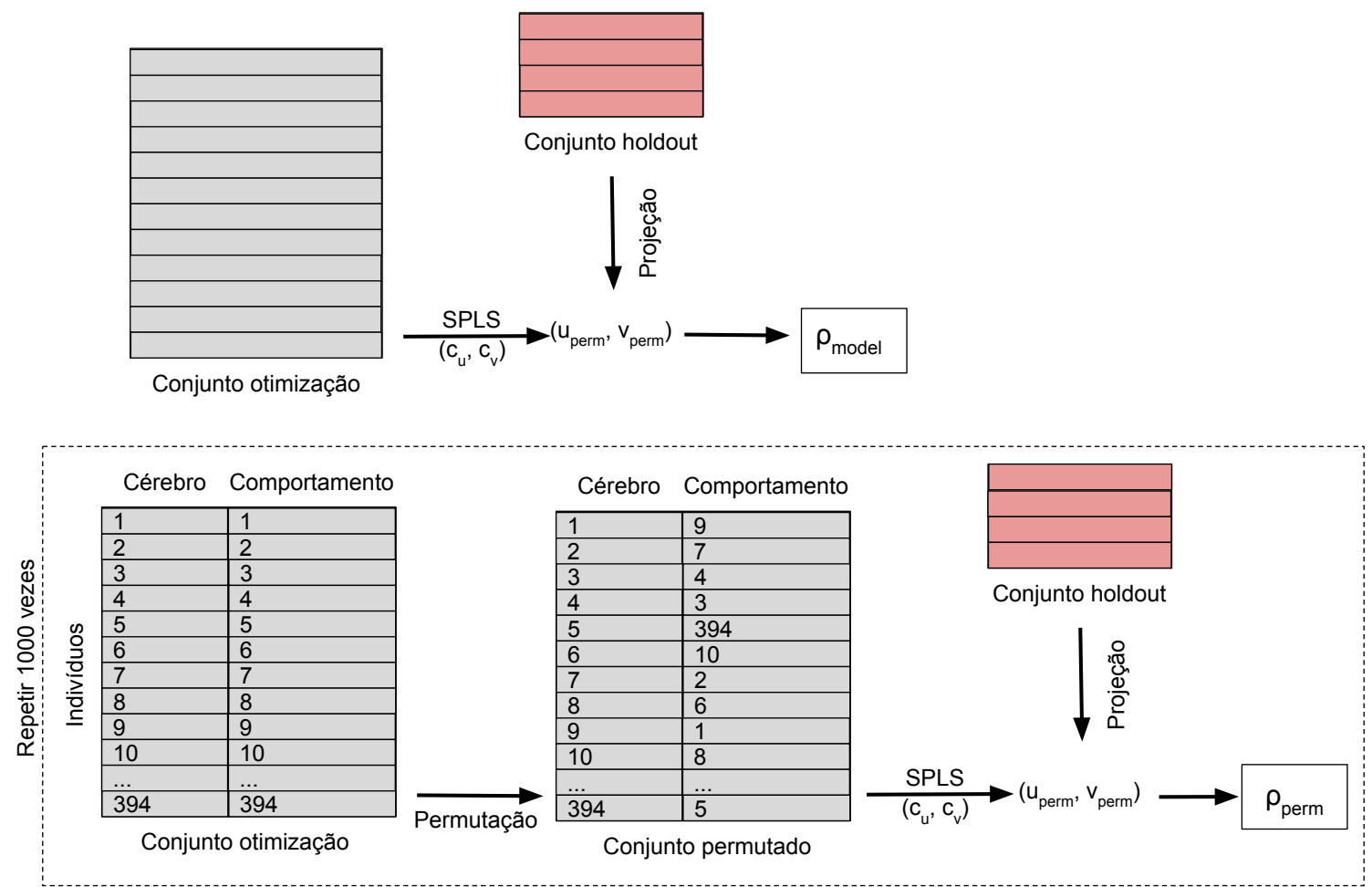

Figura 3.4: Treinamos o SPLS com os parâmetros de regularização selecionados no conjunto de otimização e obtemos vetores de pesos. Obtemos os scores projetando o conjunto holdout nos vetores de pesos. A correlação out-of-sample $\rho_{\text {model }}$ será a correlação entre esses vetores de scores. Permutamos os dados de comportamento para quebrar a associação entre os dados dos indivíduos, treinamos o SPLS com os parâmetros de regularização selecionados e projetamos o mesmo conjunto holdout nos vetores de pesos para obter os vetores de scores dos dados permutados. Calculamos a correlação outof-sample dos dados permutados como a correlação entre esses vetores de scores. Repetimos o processo de permutação 1000 vezes e calculamos o p-valor conforme a equação 3.20 .

Os vetores de pesos obtidos nesta associação serão usados para a deflação dos dados e então, repetimos todo o processo para verificar se existe um segundo modo de associação cérebro-comportamental estatisticamente significativo.

\section{Deflação dos dados}

Se a hipótese omnibus for rejeitada (Seção 3.2.8), então o modo de associação cérebrocomportamental encontrado pelo SPLS é estatisticamente significativo. Portanto, precisamos remover este efeito dos dados antes de verificar se existe uma segunda associação. Este processo é feito através da deflação das matrizes de dados X e Y, removendo os efeitos descritos pelos vetores de pesos $\mathbf{u}$ e $\mathbf{v}$ referentes à primeira associação encontrada.

Queremos garantir a ortogonalidade entre os pares de vetores de pesos encontrados em cada modo de associação. Pois, caso os vetores não sejam ortogonais, podemos encontrar o mesmo efeito (como por exemplo um efeito de idade ou a mesma região do cérebro) representado em mais de um modo de associação (Monteiro, RAO, Ashburner et al., 2016). Para isso, utilizamos o método de "projection deflation" previamente descrito para o método Sparse PCA (Partial Component Analysis) (MACKey, 2008). Este método remove 
o efeito explicado por $\mathbf{u}$ e $\mathbf{v}$ de $\mathrm{X}$ e $\mathrm{Y}$ removendo de cada matriz de dados a sua projeção no espaço gerado por seu respectivo vetor de pesos. Assim, sendo $i$ o i-ésimo efeito de associação a ser avaliado nos dados $\mathbf{X}_{i}$ e $\mathbf{Y}_{i}$, temos:

$$
\mathbf{X}_{i+1}=\mathbf{X}_{i}\left(\mathbf{I}-\mathbf{u}_{i} \mathbf{u}_{i}^{\top}\right)
$$

$\mathrm{e}$

$$
\mathbf{Y}_{i+1}=\mathbf{Y}_{i}\left(\mathbf{I}-\mathbf{v}_{i} \mathbf{v}_{i}^{\top}\right)
$$

Utilizando os dados $\mathbf{X}_{i+1}$ e $\mathbf{Y}_{i+1}$ após a remoção do efeito, repetimos os passos de otimização do modelo (seção 3.2.8) e avaliação do novo efeito encontrado (seção 3.2.8). Repetimos este processo até que um efeito de associação não seja estatisticamente significativo ou até obtermos todos os $d$ modos de associação entre as variáveis, como descrito em 3.2.6.

\subsection{Resultados}

O método SPLS usado na análise ajusta o modelo a 10 diferentes divisões dos dados (sorteios de indivíduos para o conjunto otimização e para o conjunto holdout), apresentamos aqui os resultados para a divisão que apresentou a menor p-valor e maior correlação outof-sample, conforme os critérios descritos em 3.2.8. Identificamos um modo de associação cérebro-comportamental com alta significância (correlação no conjunto holdout $=0.3830$, pvalor $<0.01$, corrigido por Bonferroni para 10 divisões dos dados). Após a deflação dos dados, a segunda associação obtida não obteve significância estatística e por isso não reportamos resultados para outras associações. Para examinar como a relação cérebro-comportamento identificada é expressa em toda a amostra, criamos um gráfico de dispersão de $\mathbf{P}_{X}$ contra $\mathbf{P}_{Y}$ (scores cérebro $\times$ comportamento) mostrado na Figura 3.5. Embora as informações de diagnóstico não tenham sido inseridas no modelo, colorindo os indivíduos com DT em vermelho e os indivíduos com TEA em azul mostra que os scores capturaram uma relação com o diagnóstico tanto na dimensão do cérebro quanto na dimensão do comportamento. Na Figura 3.5, o gráfico mostra que o grupo de DT está localizado principalmente na parte inferior do gráfico, enquanto o grupo TEA está espalhado pela diagonal apresentando uma maior variação nos scores de cérebro e comportamento, o que está de acordo com a "natureza espectral" do distúrbio. A sobreposição dos grupos de diagnóstico no gráfico é pequena, o que indica que a associação identificada captura as diferenças entre os grupos de DT e TEA.

O método SPLS encontra $\mathbf{u}$ and $\mathbf{v}$ que maximiza a covariância entre $\mathbf{P}_{X}$ e $\mathbf{P}_{Y}$. Para obter valores relativos ao envolvimento do conjunto original de conexões cerebrais e variáveis comportamentais, calculamos a correlação de $\mathbf{P}_{X}$ e $\mathbf{P}_{Y}$ com cada variável dos conjuntos originais de dados $\mathrm{X}$ e $\mathrm{Y}$, respectivamente, obtendo $\mathbf{u}^{\prime}$ e $\mathbf{v}^{\prime}$. Assim, obtemos um coeficiente de correlação para cada uma das variáveis cerebrais e comportamentais, mesmo para aquelas que não foram selecionadas pela abordagem esparsa. Os coeficientes de correlação $\mathbf{v}^{\prime}$ para as variáveis comportamentais são apresentados na Figura 3.6. As variáveis de comportamento com coeficientes de correlação mais altos apresentadas na Figura 3.6 estão relacionadas aos sintomas de TEA, então podemos esperar que as conexões cerebrais com alto coeficiente de correlação também tenham relação com o TEA. As variáveis originais 


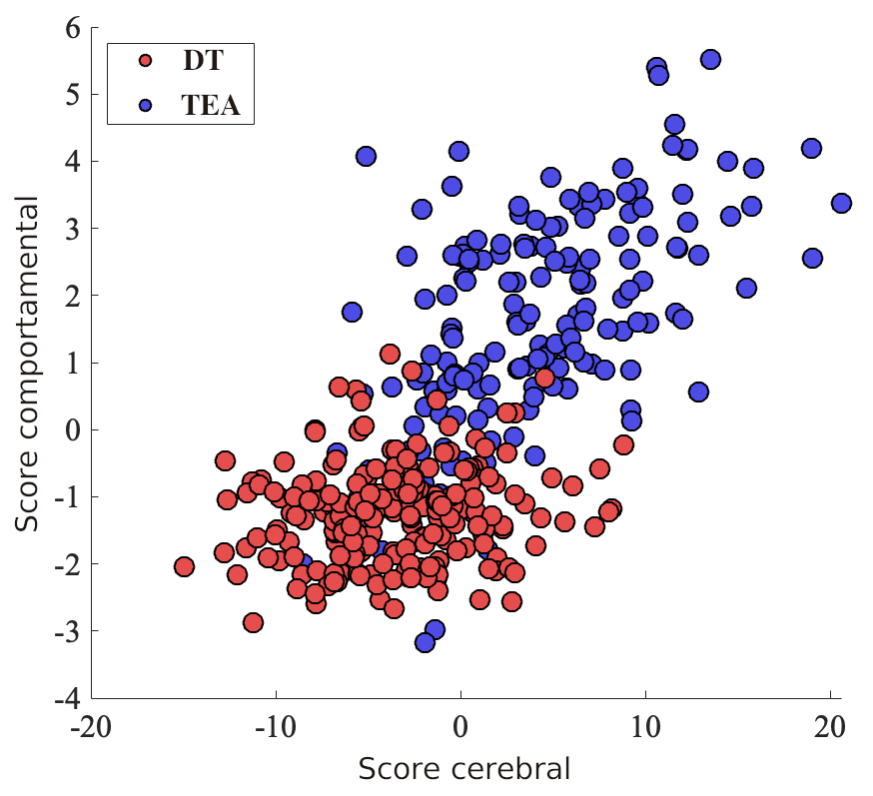

Figura 3.5: Associação cérebro-comportamental representada por um gráfico de dispersão em que cada indivíduo (ponto no gráfico) é representado pelo score do cérebro no eixo x e pelo score do comportamento no eixo y. O ponto que representa cada indivíduo foi colorido de acordo com seu grupo de diagnóstico (vermelho para DT e azul para TEA).

selecionadas pelo SPLS são apresentadas na Figura 3.7.

Para facilitar a análise dos resultados de conectividade cerebral, dividimos o cérebro em redes funcionais usando um atlas obtido por clusterização espectral dos dados de fMRI em estado de repouso do ABIDE 1 (SATo et al., 2016). Como a conectividade de nossa população é representada pela correlação de Pearson, cada conectividade do cérebro pode ter um sinal positivo ou negativo. Assim, o sinal dos coeficientes de correlação u' não representa a direção das diferenças. Para representar o "aumento" ou "diminuição" da conectividade em relação às variáveis comportamentais selecionadas, seguimos os passos a seguir. Multiplicamos cada elemento do vetor $\mathbf{u}^{\prime}$ (coeficientes de correlação do cérebro) pelo sinal do valor médio de conectividade de cada aresta na população, criando um novo vetor $\mathbf{u}^{\prime \prime}$ de "variação da conectividade". Convertemos o vetor em uma matriz de tamanho $316 \times 316$ usando a função matlab "reshape". Usamos essa matriz como uma entrada para o software BrainNet Viewer (M. XIA et al., 2013) para gerar o gráfico de conectividade na Figura 3.8 A e B mostrando os 100 valores mais altos em vermelho e os 100 valores mais baixos em azul. Também criamos dois gráficos de resumo de conectividade por nós (cada nó representa uma ROI). Para o gráfico de "aumento" mostrado na Figura 3.8 C, consideramos um subgrupo das 100 arestas com valores mais altos. Para cada nó, somamos todos os valores das arestas do subgrupo conectado ao nó. O mesmo processo foi repetido para criar o gráfico de "diminuição" mostrado na Figura 3.8 D considerando as 100 arestas com valores mais baixos. Observe que um nó pode estar presente em ambos os gráficos de aumento e diminuição, mas apenas algumas pequenas áreas apresentam sobreposição. Na figura 3.8 A, podemos ver que conexões diminuídas são principalmente de longa distância, ocorrendo entre nós pertencentes ao modo de rede padrão. Também encontramos conexões de curto alcance diminuídas entre nós da rede somatomotora que são predominantemente inter- 


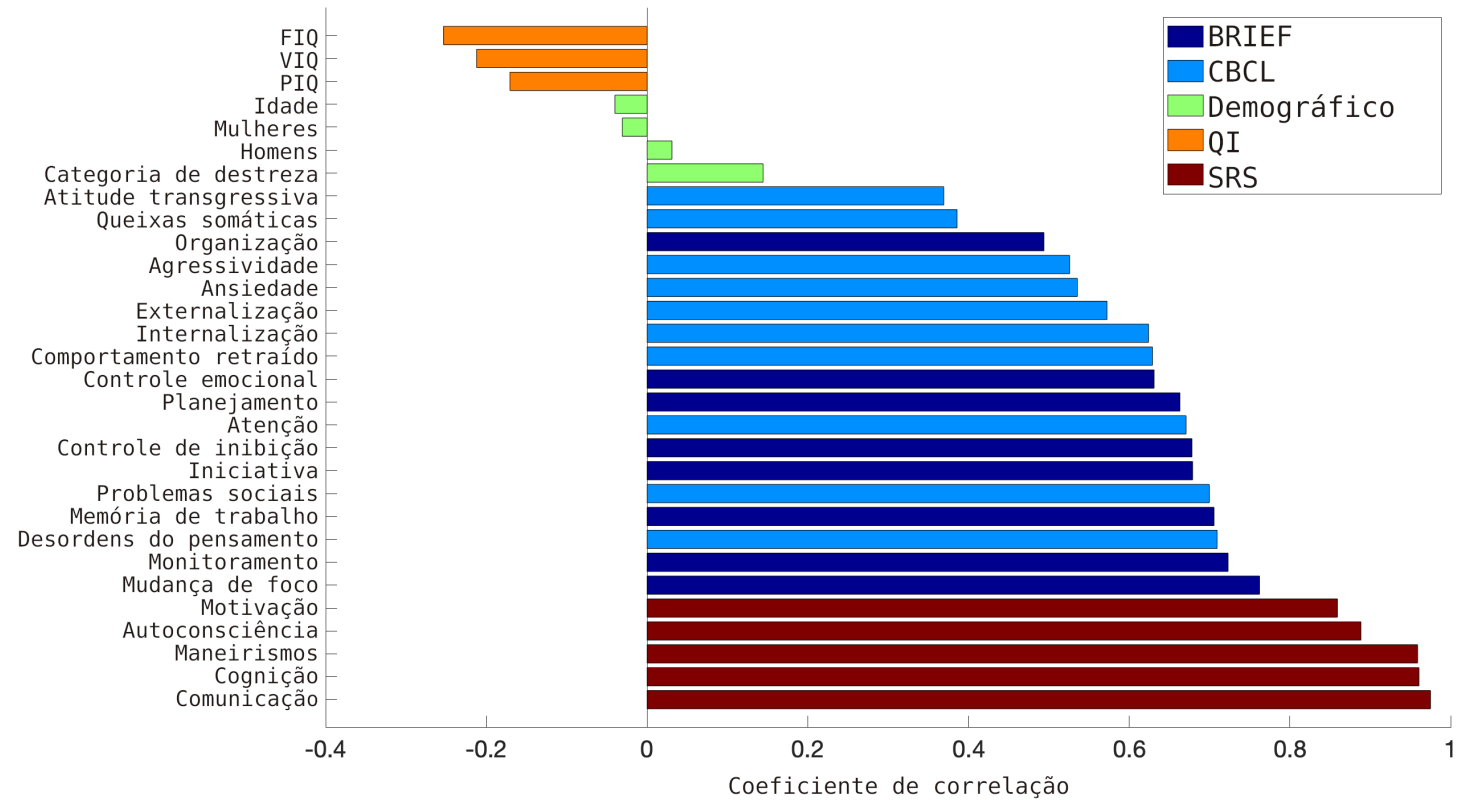

Figura 3.6: Correlações entre as variáveis comportamentais originais e os scores de comportamento do sujeito obtidos pelo SPLS. As variáveis com maior coeficiente de correlação pertencem à escala SRS que mede características de responsividade social.

hemisféricas. Na figura $3.8 \mathrm{~B}$, podemos ver o aumento das conexões principalmente entre os nós pertencentes à rede somatomotora e a rede visual. Nas Figuras 3.8 C e D resumindo as conexões por nó, podemos ver que os nós mais envolvidos em conexões diminuídas (Figura $3.8 \mathrm{C}$ ) pertencem às redes de modo padrão, somatomotora e frontoparietal e os nós mais envolvidos em conexões aumentadas (Figura 3.8 D) pertencem às redes somatomotora, modo padrão, visual e cerebelar.

Para mostrar como a conectividade funcional inter-redes varia na amostra, usamos a variação da conectividade funcional $\mathbf{u}^{\prime \prime}$ para calcular uma matriz da porcentagem de conexões entre cada par de redes mostrado na Figura 3.9. Novamente consideramos apenas as 100 arestas mais negativamente correlacionadas e os 100 arestas mais positivamente correlacionados para criar cada matriz mostrada nos painéis A e B, respectivamente. Já que estamos usando $\mathbf{u}^{\prime \prime}$, o painel A representa conexões mais fracas para indivíduos de score alto e conexões mais fortes para indivíduos de score baixo (gráfico de conectividade diminuída) e o painel B representa conexões mais fortes para indivíduos de score alto e conexões mais fracas para indivíduos de score baixo (gráfico de conectividade aumentada). No painel A, a porcentagem das 100 principais conexões negativamente correlacionadas entre as redes é de $54 \%$ na conectividade intra-rede no modo padrão, $23 \%$ na conectividade intra-rede somatomotora e $10 \%$ entre o modo padrão e inter-rede frontoparietal conectividade. No painel $\mathrm{B}$, podemos ver que a porcentagem das 100 principais conexões negativamente correlacionadas inter-redes é de $49 \%$ entre as redes somatomotora e visual, $16 \%$ entre o as redes de modo padrão e somatomotora, $8 \%$ intra-rede cerebelar e $7 \%$ entre as redes cerebelar e de modo padrão. As outras combinações de conectividade de rede representam menos de $5 \%$ cada, portanto, as excluímos de uma análise posterior. 
3.3 | RESULTADOS

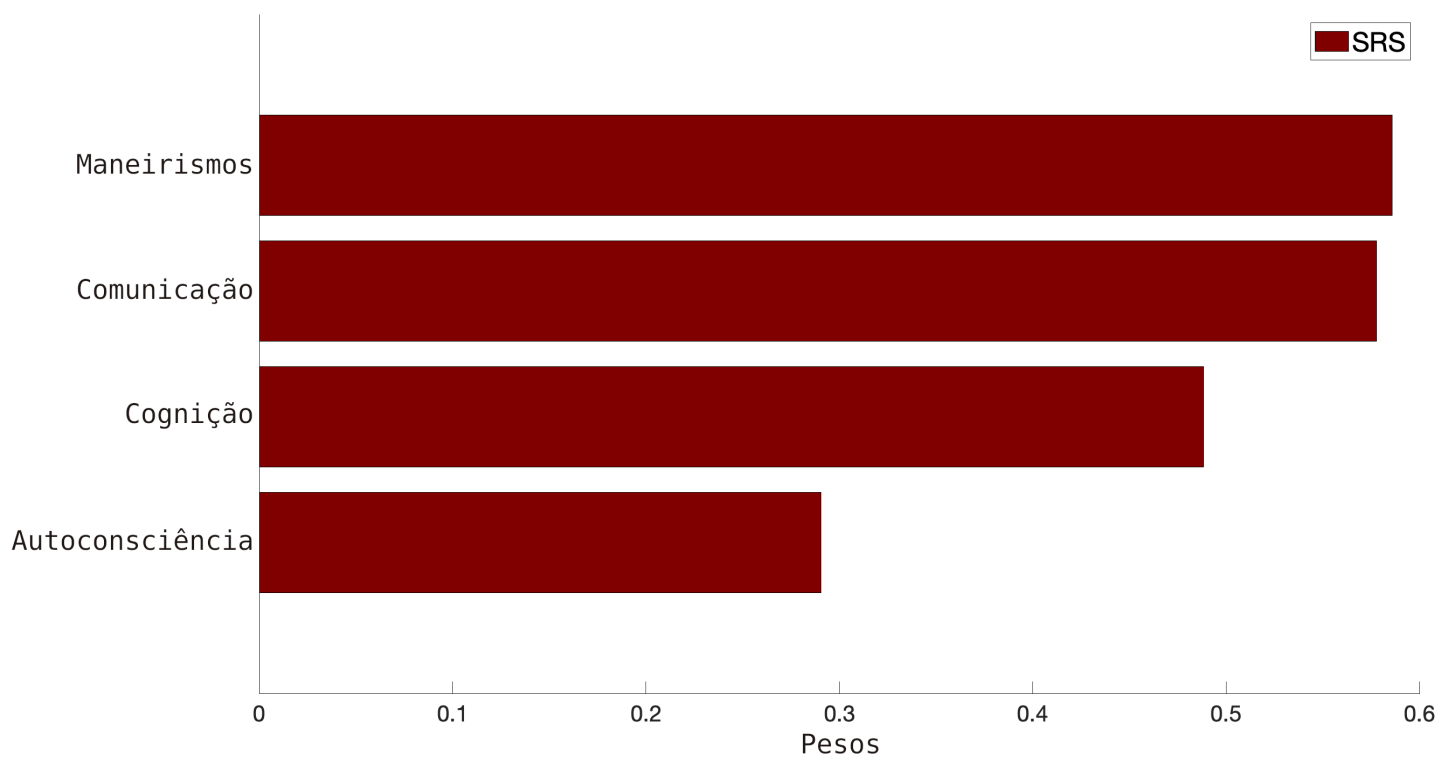

Figura 3.7: Pesos das variáveis de comportamento selecionadas pelo SPLS. O método selecionou apenas quatro variáveis de comportamento, todas pertencentes à SRS. São elas: maneirismos, comunicação, cognição e autoconsciência. 
A

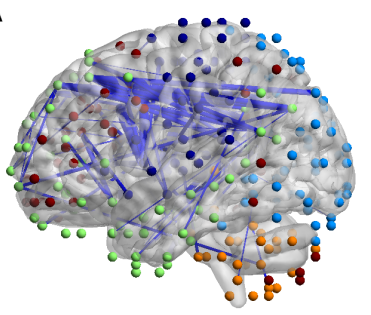

B

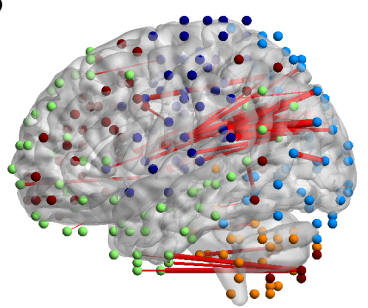

C

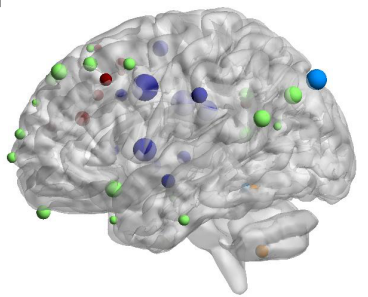

D

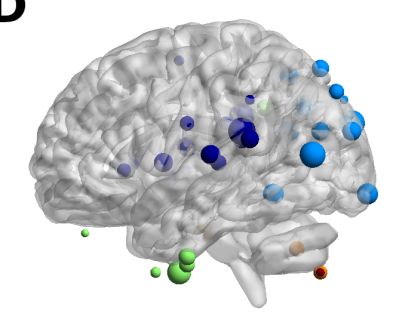

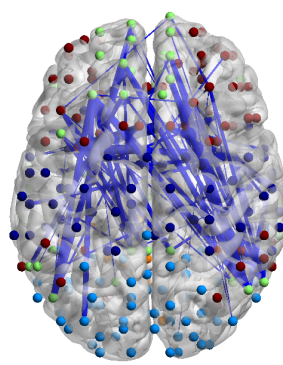
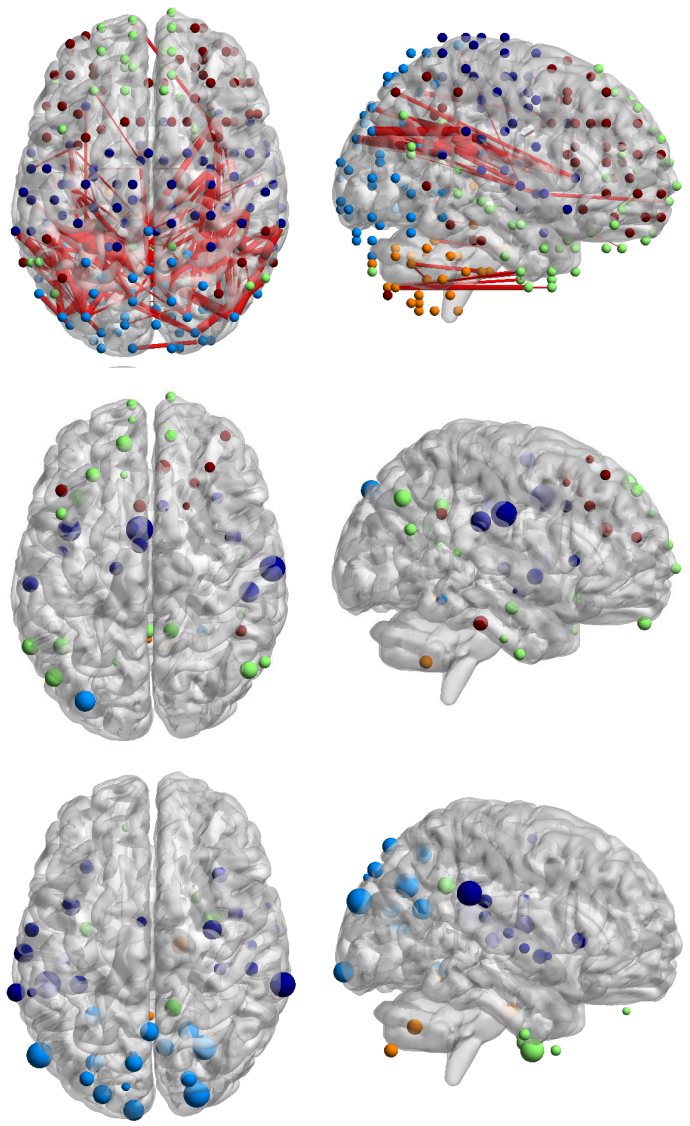

Frontoparietal

Somatomotora

Figura 3.8: Representação da variação da conectividade cerebral em visão sagital (esquerda e direita) e axial (meio). (A) As primeiras 100 conexões cerebrais mais negativamente correlacionadas à associação cérebro-comportamental obtida pelo SPLS. Para fins de interpretabilidade, a correlação de cada conexão foi multiplicada pelo sinal da conexão média da população. Assim, este mapa representa conexões mais fracas para indivíduos com score cerebral alto e conexões mais fortes para indivíduos de score cerebral baixo. A espessura das arestas é proporcional à correlação absoluta. (B) As primeiras 100 conexões cerebrais mais positivamente correlacionadas à associação cérebro-comportamental obtida pelo SPLS. Este mapa representa conexões mais fortes para indivíduos com score cerebral alto $e$ conexões mais fracas para indivíduos com score cerebral baixo. (C) Resumo por nó das primeiras 100 conexões cerebrais mais negativamente correlacionadas à associação cérebro-comportamental obtida pelo SPLS. O tamanho do nó é proporcional à correlação absoluta média. (D) Resumo por nó das primeiras 100 conexões cerebrais mais positivamente correlacionadas à associação cérebro-comportamental obtida pelo SPLS. Em todos os 4 painéis, a cor do nó representa a rede funcional a eles atribuída de acordo com (SATO et al., 2016). 


\section{A \\ Cerebellar Network Default-Mode Network Fronto-Parietal Network Somatomotor Network Visual Network}

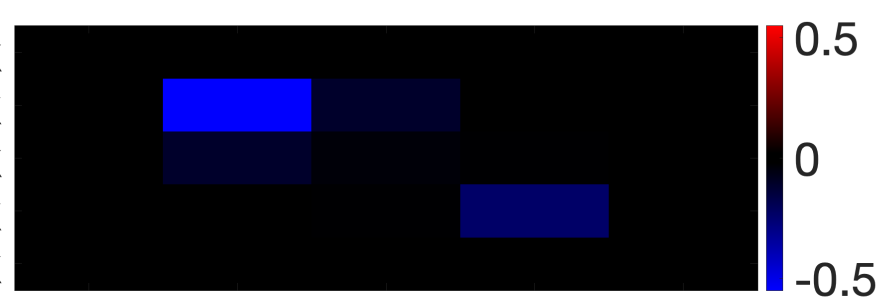

\section{B}

Cerebellar Network Default-Mode Network Fronto-Parietal Network Somatomotor Network Visual Network
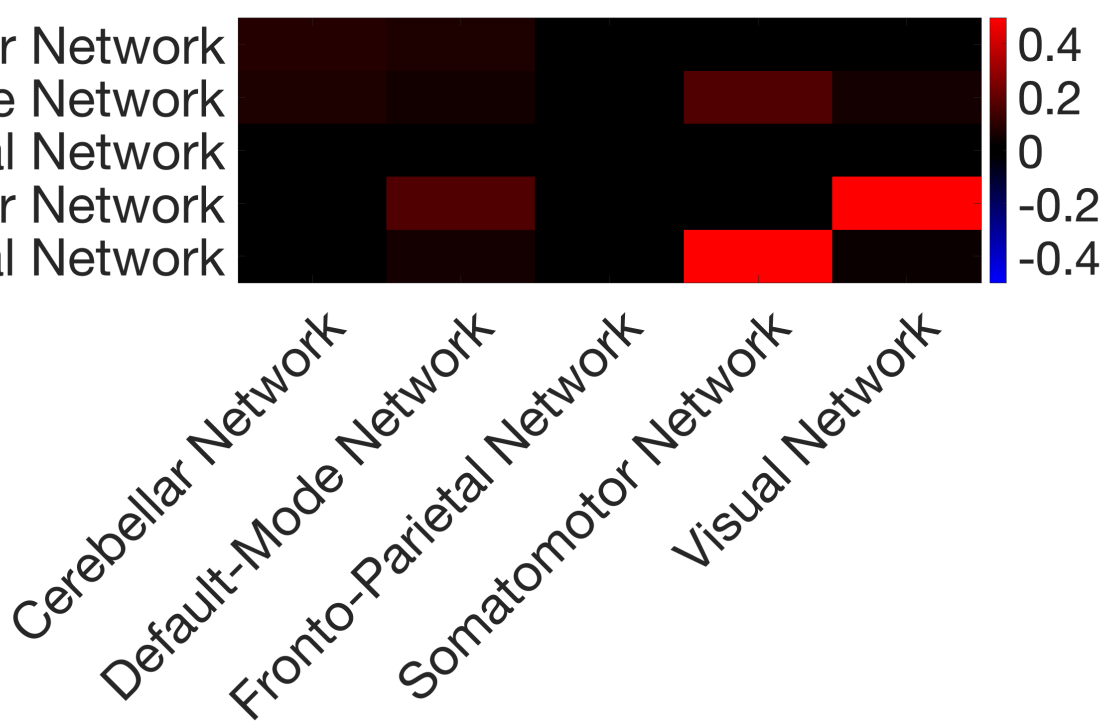

Figura 3.9: Conectividade funcional entre redes. Os painéis ( $A, B)$ representam a porcentagem de conexões que ocorrem entre cada par de redes funcionais. Consideramos as 100 conexões mais negativamente e mais positivamente correlacionadas para os painéis $A$ e $B$, respectivamente. A matriz triangular inferior soma 1 (100\%). 


\subsection{Discussão}

No presente estudo nosso objetivo era encontrar uma associação cérebrocomportamental multivariada relacionando conexões cerebrais funcionais e características comportamentais de forma que essa associação se expresse diferentemente no grupo com DT e no grupo com TEA. Nós identificamos um modo de associação estatisticamente significativo através de um espaço latente que maximiza a associação multivariada entre as conexões cerebrais e as variáveis de comportamento. As conexões cerebrais mais envolvidas na associação identificada estão localizadas principalmente na rede de modo padrão e entre as redes somatomotora e visual. As variáveis de características comportamentais com maior peso representam medidas de responsividade social. A associação identificada se expressa diferentemente nos grupos de DT e TEA (Ver Figura 3.5) sugerindo um possível papel das conexões cerebrais envolvidas e variáveis comportamentais no TEA.

As pesquisas em neurociência geralmente estão interessadas em entender a relação entre diferentes modalidades de medidas, como dados de imagens cerebrais, escalas psicométricas ou informações demográficas. A abordagem mais frequentemente utilizada é realizar uma análise univariada entre uma medida de resumo da condição de interesse com cada uma das medidas das modalidades a serem analisadas e corrigir os resultados para comparações múltiplas. Esta abordagem não considera a relação conjunta entre as diferentes modalidades de dados. Nos últimos 10 anos, um número crescente de estudos tem aplicando métodos multivariados para estudar essas relações (S. SMITH et al., 2015; DrysDALE et al., 2017; Dominik A. Moser et al., 2018; C. H. XiA et al., 2018; Mihalik, Ferreira, Rosa et al., 2019). Uma das principais vantagens sobre uma abordagem univariada, como a regressão linear, é a possibilidade de correlacionar um grupo de variáveis que descrevem a atividade cerebral ou traços morfológicos com um grupo de medidas de comportamento. Dessa forma, podemos caracterizar melhor o transtorno considerando um conjunto de aspectos de um mesmo modelo. No contexto da pesquisa de TEA, os estudos de conectividade funcional do cérebro geralmente focam apenas nas diferenças de conectividade do cérebro entre grupos (RAmos et al., 2019) ou padrões de conectividade correlacionados com medidas comportamentais post-hoc (TANG et al., 2020). Em nosso estudo, selecionamos os pesos das conexões enquanto consideramos as medidas comportamentais no mesmo modelo.

Nossos resultados mostram conectividade diminuída em indivíduos com scores mais altos (majoritariamente composto pelo grupo TEA) principalmente nas redes de modo padrão e somatomotora. Os resultados indicam conectividade aumentada em indivíduos de scores mais altos (majoritariamente grupo TEA) principalmente entre as redes somatomotora e visual e entre as redes somatomotora e de modo padrão. Nossos resultados estão de acordo com a literatura atual de diferenças observadas na conectividade funcional em estado de repouso em TEA. A diminuição da conectividade na rede de modo padrão associada a dificuldades de interação social em TEA foi relatada anteriormente (AssAF et al., 2010; Аввотт et al., 2016). Estudos anteriores incluindo pacientes do ABIDE II também relataram associações de dificuldade de interação social com conectividade diminuída na rede do modo padrão e conectividade inter-rede aumentada entre as redes de modo padrão e rede somatomotora (WANG et al., 2019; TANG et al., 2020). A conectividade intra-rede diminuída na rede somatomotora também é relatada (TANG et al., 2020). O aumento da conectividade entre a rede sensório-motora e a rede visual foi anteriormente associado à 
gravidade dos sintomas de TEA em crianças pequenas (CHEN et al., 2020).

Em relação aos dados de comportamento, podemos observar que todas as variáveis obtidas pelas escalas psicométricas apresentam coeficientes de correlação superiores às variáveis demográficas (Figura 1B). Isso significa que a associação cérebro-comportamental encontrada pelo SPLS está mais relacionada às características comportamentais do que à categoria de idade, sexo ou destreza. As variáveis com maiores coeficientes de correlação foram obtidas pelo formulário SRS. Embora o SRS não tenha sido desenvolvido para ser um instrumento de diagnóstico de autismo por si só (DuKu et al., 2013), ele é frequentemente aplicado como parte de uma avaliação diagnóstica abrangente de TEA e é considerado uma medida quantitativa válida de traços autistas (Constantino et al., 2003). O SRS caracteriza o comprometimento do comportamento social por meio de 5 medidas principais: comunicação social, cognição social, maneirismos, autoconsciência e motivação. Embora o CBCL tenha apresentado baixa acurácia discriminativa para TEA, foi associado a questões emocionais e comportamentais, que podem ser consideradas complementares à análise de traços de autismo (HAVDAHL et al., 2016). Crianças com TEA também apresentam déficits significativos nas funções executivas (GRANADER et al., 2014), portanto, as variáveis obtidas pelo BRIEF, que mede comportamentos associados à função executiva, podem ajudar a caracterizar este grupo.

Uma das principais limitações deste estudo é a falta de um padrão para as escalas psicométricas usadas nos locais de coleta. O projeto ABIDE II contém uma grande amostra de dados (1114 indivíduos), porém, cada laboratório fez a coleta de acordo com seus próprios critérios de pesquisa. Ou seja, não existe conformidade sobre quais escalas psicométricas seriam preenchidas pelo participante no momento da coleta. Assim, foi necessário selecionar uma subamostra de participantes para a qual foram preenchidas ao menos duas das três escalas escolhidas para esta pesquisa. Pelo mesmo motivo, não pudemos analisar como a severidade do TEA está relacionada à associação cérebro-comportamental encontrada, uma vez que não temos informações sobre severidade para a maioria dos indivíduos selecionados na subamostra. Além disso, devido à necessidade de restrição de movimento e tolerância ao ruído da máquina, nossa amostra pode estar viesada para representar mais sujeitos com sintomas sensoriais leves. Finalmente, embora tenhamos usado uma estrutura de múltiplos holdouts, o ideal seria usar uma amostra independente para validar nosso modelo.

Em resumo, nosso estudo conseguiu identificar uma dimensão latente de associação cérebro-comportamental que é expressa diferentemente entre os grupos DT e TEA, havendo pouca sobreposição entre os grupos no gráfico de dispersão dos scores dos sujeitos. As principais variáveis comportamentais envolvidas na associação identificada estão relacionadas ao comprometimento social, como déficits na comunicação social, cognição social e maneirismos. Os principais resultados cerebrais são a diminuição da conectividade funcional na rede de modo padrão e rede somatomotora e o aumento da conectividade funcional entre as redes somatomotora e visual e entre as redes somatomotora e de modo padrão no TEA. Nossos achados sugerem uma relação entre as redes cerebrais identificadas e deficiências sociais em TEA. Além disso, a dispersão diferenciada dos grupos na dimensão latente com pouca sobreposição sugere que a análise multivariada combinando medidas multimodais, como cérebro e comportamento, é uma técnica promissora para elucidar diferenças entre os diagnósticos. 


\subsection{Conclusão}

Detectamos um modo de associação multivariada estatisticamente significativa entre a conectividade de fMRI em estado de repouso e informações fenotípicas e demográficas em crianças com DT e crianças com TEA. Nossos resultados são consistentes em todos os conjuntos de validação testados e devem generalizar para novos dados. 


\section{Capítulo 4}

\section{Considerações finais}

Nossa proposta neste trabalho foi estudar semelhanças e diferenças entre indivíduos analisando a conectividade funcional cerebral. Para isso, nos focamos em duas abordagens: (i) agrupar indivíduos semelhantes e (ii) posicionar os indivíduos em um espectro estratificado do diagnóstico.

Nossa solução para (i) foi modelar as conectividades funcionais de cada indivíduo como um grafo e fazer a clusterização desses grafos. Nossa principal contribuição para esta abordagem foi o desenvolvimento de dois algoritmos de clusterização para grafos baseados em análise espectral: um k-means para grafos do mesmo tamanho e um algoritmo de clusterização por Expectativa-Maximização para grafos de tamanhos diferentes chamado gCEM. Nós incluímos os algoritmos desenvolvidos em uma nova versão do pacote statGraph do R disponível no CRAN (https://CRAN.R-project.org/package=statGraph). Os algoritmos k-means e gCEM correspondem às funções kmeans . graph e gCEM do pacote, respectivamente.

Nossa principal contribuição para (ii) foi mostrar que o método SPLS utilizando framework de múltiplos holdouts desenvolvido por Mihalik, Ferreira, Moutoussis et al., 2020 consegue elucidar uma dimensão de variação de cérebro e comportamento que reflete a natureza espectral do diagnóstico de TEA. Nosso resultado do espalhamento dos indivíduos no espectro representados no plot 3.5 estão de acordo com a literatura ao mostrar uma variação maior nos indivíduos com TEA tanto na dimensão de cérebro como na dimensão de comportamento. Até onde sabemos, este é o primeiro estudo utilizando SPLS com framework de múltiplos holdouts a mostrar uma clara diferença na variação entre os grupos de diagnóstico tanto na dimensão do cérebro como na dimensão do comportamento. Do ponto de vista do método recém proposto, este achado é importante para confirmar seu potencial para estudo de estratificação de diagnósticos utilizando múltiplas modalidades de dados. Nossa contribuição para a área de estudos de TEA é uma evidência de que a hipótese de um espectro de variação do diagnóstico se confirma não só na observação comportamental, mas parece ser também observável na conectividade funcional do cérebro.

No trabalho desenvolvido em (i) nós mostramos por simulação que as medidas de divergência entre os espectros dos grafos nos permitem diferenciar os clusters conforme 
a diferença na organização da conectividade. Mostramos que mesmo quando os clusters apresentam o mesmo número de vértices, arestas e distribuição de graus, conseguimos diferenciar os clusters enquanto os grafos apresentavam diferenças na organização das arestas. Uma possível extensão deste trabalho seria obter uma demonstração da relação dos autovalores do grafo com a organização de suas arestas. Também seria interessante obter uma normalização das densidades espectrais que possibilitassem comparar diretamente espectros de grafos de tamanhos diferentes de qualquer modelo.

No trabalho desenvolvido em (ii) nós utilizamos uma subamostra do banco de dados ABIDE para os quais nós tivéssemos informações de pelo menos duas das três escalas psicométricas utilizadas. Esta limitação nos gerou um grupo de estudos bastante reduzido e sem disponibilidade de informações adicionais como grau de severidade do autismo. Uma possível extensão deste trabalho seria avaliar uma amostra maior de indivíduos com mais dados de escalas psicométricas. Isso nos possibilitaria avaliar a relação do espectro com a severidade dos sintomas e a existência de subgrupos de autismo com características comportamentais em comum. 


\section{Capítulo 5}

\section{Contribuições}

\subsection{Produção de textos técnicos e científicos}

Este trabalho contribui para a análise de diferenças entre grupos de diagnóstico com base em conectividade funcional. Apresentamos dois métodos de classificação não supervisionada (clusterização) modelando a conectividade funcional como um grafo e um método de classificação supervisionada (machine learning) que utiliza dados de cérebro e comportamento para fazer a classificação. A seguir listamos trabalhos que foram ou serão publicados a partir dos resultados.

\subsubsection{Artigo publicado}

Inicialmente fizemos um estudo de conectividades funcionais anormais entre o córtex cerebral e o cerebelo em pessoas com transtorno do espectro autista. Os resultados foram descritos no seguinte artigo:

- Ramos et al. (2019) Taiane Coelho Ramos, Joana Bisol Balardin, João Ricardo Sato e André Fujita. "Abnormal cortico-cerebellar functional connectivity in autism spectrum disorder." Frontiers in systems neuroscience, v. 12, p. 74, 2019.

\subsubsection{Artigo submetido}

Os algoritmos de clusterização propostos neste trabalho e resultados das aplicações em dados reais foram compilados no seguinte trabalho "Spectral density based clustering algorithms for complex networks", de Taiane Coelho Ramos, Janaína Mourão-Miranda e André Fujita, sob revisão.

\subsubsection{Artigos em preparação}

Os algoritmos de clusterização de grafos propostos neste trabalho foram incluídos em uma biblioteca de métodos estatísticos em grafos, juntamente com novos métodos de estatística em grafos desenvolvidos pelo laboratório. Um artigo descrevendo a última versão disponível da biblioteca e todos os seus métodos está atualmente em preparação. 
Com base nos resultados na análise da relação entre variáveis comportamentais e fenotípicas e variáveis de conectividade cerebral em indivíduos com e sem autismo, estamos preparando um manuscrito para publicação.

\subsection{Software}

Os algoritmos de clusterização de grafos propostos estão disponíveis no CRAN no pacote statGraph (https://CRAN.R-project.org/package=statGraph), funções kmeans.graph e gCEM, sob licença GNU GPL. 


\section{Referências}

[Аввотт et al. 2016] Angela E. Аввотт et al. "Patterns of Atypical Functional Connectivity and Behavioral Links in Autism Differ Between Default, Salience, and Executive Networks”. en. Em: Cerebral Cortex 26.10 (out. de 2016), pgs. 4034-4045. ISSN: 1047-3211. DOI: 10.1093/cercor/bhv191. URL: https://academic.oup.com/ cercor/article/26/10/4034/2389082 (acesso em 09/07/2020) (citado na pg. 48).

[Achenbach 2011] Thomas M. Achenbach. "Child Behavior Checklist". en. Em: Encyclopedia of Clinical Neuropsychology. Ed. por Jeffrey S. Kreutzer, John DeLucA e Bruce CAPLAN. New York, NY: Springer, 2011, pgs. 546-552. ISBN: 9780387799483. DOI: 10.1007/978-0-387-79948-3_1529. URL: https://doi.org/10.1007/978-0-38779948-3_1529 (acesso em 23/10/2020) (citado nas pgs. 28, 31).

[Allen et al. 2004] Greg Allen, Ralph-Axel Müller e Eric Courchesne. "Cerebellar function in autism: Functional magnetic resonance image activation during a simple motor task". en. Em: Biological Psychiatry 56.4 (ago. de 2004), pgs. 269278. ISSN: 0006-3223. DOI: 10.1016/j. biopsych.2004.06.005. URL: http://www. sciencedirect.com/science/article/pii/S0006322304006511 (acesso em 28/01/2021) (citado na pg. 1).

[AN et al. 2018] Jingzhi AN et al. "Automated Assessment of Loss of Consciousness Using Whisker And Paw Movements During Anesthetic Dosing in Head-Fixed Rodents". Em: 2018 40th Annual International Conference of the IEEE Engineering in Medicine and Biology Society (EMBC). ISSN: 1557-170X. Jul. de 2018, pgs. 730-733. DOI: 10.1109/EMBC.2018.8512377 (citado na pg. 18).

[Assaf et al. 2010] Michal Assaf et al. "Abnormal functional connectivity of default mode sub-networks in autism spectrum disorder patients”. en. Em: NeuroImage 53.1 (out. de 2010), pgs. 247-256. ISSN: 1053-8119. DOI: $10.1016 /$ j. neuroimage. 2010 . 05 . 067. URL: http : / / www . sciencedirect . com / science / article / pii / S1053811910008013 (acesso em 09/07/2020) (citado na pg. 48).

[Association 2013] American Psychiatric Association. Diagnostic and Statistical Manual of Mental Disorders (DSM-5®). en. American Psychiatric Pub, mai. de 2013. ISBN: 978-0-89042-557-2 (citado na pg. 27). 
[BarabÁsi e Albert 1999] Albert-László BarabÁsi e Réka Albert. "Emergence of scaling in random networks". Em: Science 286.5439 (1999), pgs. 509-512 (citado nas pgs. 12, 13).

[Bellec et al. 2016] Pierre Bellec et al. "The Neuro Bureau ADHD-200 Preprocessed repository”. Em: NeuroImage 144 (jul. de 2016). Dor: 10.1016/j.neuroimage.2016. 06.034 (citado na pg. 29).

[Biswal et al. 1995] B. Biswal, F. Z. Yetkin, V. M. Haughton e J. S. Hyde. "Functional connectivity in the motor cortex of resting human brain using echo-planar MRI". eng. Em: Magnetic Resonance in Medicine 34.4 (out. de 1995), pgs. 537-541. IssN: 0740-3194 (citado na pg. 28).

[Blackwell et al. 2007] Paul Blackwell, Mark Edmondson-Jones e Jonathan JorDAN. Spectra of adjacency matrices of random geometric graphs. University of Sheffield. Department of Probability e Statistics, 2007 (citado na pg. 16).

[Bollobás e Béla 2001] Béla Bollobás e Bollobás BÉla. Random graphs. 73. Cambridge university press, 2001 (citado nas pgs. 12, 13).

[BRANDEs et al. 2008] Ulrik BrANDEs et al. "On modularity clustering”. Em: IEEE transactions on knowledge and data engineering 20.2 (2008), pgs. 172-188 (citado na pg. 5).

[Celeux e Govaert 1992] Gilles Celeux e Gérard Govaert. “A classification em algorithm for clustering and two stochastic versions". Em: Computational statistics \& Data analysis 14.3 (1992), pgs. 315-332 (citado nas pgs. 1, 6, 9).

[Celeux e Govaert 1995] Gilles Celeux e Gérard Govaert. "Gaussian parsimonious clustering models”. Em: Pattern recognition 28.5 (1995), pgs. 781-793 (citado nas pgs. $1,6,9)$.

[CHen et al. 2020] Bosi CHen et al. "Greater functional connectivity between sensory networks is related to symptom severity in toddlers with autism spectrum disorder". eng. Em: Fournal of Child Psychology and Psychiatry, and Allied Disciplines (mai. de 2020). ISSN: 1469-7610. DoI: 10.1111/jcpp.13268 (citado na pg. 49).

[Christensen 2016] Deborah L. Christensen. "Prevalence and Characteristics of Autism Spectrum Disorder Among Children Aged 8 Years - Autism and Developmental Disabilities Monitoring Network, 11 Sites, United States, 2012”. Em: MMWR. Surveillance Summaries 65 (2016). IssN: 1546-07381545-8636. DOI: 10.15585/mmwr. ss6503a1. URL: http://www.cdc.gov/mmwr/volumes/65/ss/ss6503a1.htm (acesso em 22/01/2017) (citado na pg. 27). 
REFERÊNCIAS

[Constantino 2013] John N. Constantino. "Social Responsiveness Scale". en. Em: Encyclopedia of Autism Spectrum Disorders. Ed. por Fred R. Volkmar. New York, NY: Springer, 2013, pgs. 2919-2929. ISBN: 9781441916983. DOI: 10.1007/978-14419-1698-3_296. URL: https://doi.org/10.1007/978-1-4419-1698-3_296 (acesso em 23/10/2020) (citado nas pgs. 28, 31).

[Constantino et al. 2003] John N. Constantino et al. "Validation of a Brief Quantitative Measure of Autistic Traits: Comparison of the Social Responsiveness Scale with the Autism Diagnostic Interview-Revised". en. Em: fournal of Autism and Developmental Disorders 33.4 (ago. de 2003), pgs. 427-433. ISSN: 1573-3432. DOI: 10.1023/A:1025014929212. uRL: https://doi.org/10.1023/A:1025014929212 (acesso em 19/06/2020) (citado nas pgs. 28, 49).

[Craddock et al. 2012] R. Cameron Craddock, G. Andrew James, Paul E. HoltzheiMER, Xiaoping P. Hu e Helen S. MAYBERG. "A whole brain fMRI atlas generated via spatially constrained spectral clustering”. eng. Em: Human Brain Mapping 33.8 (ago. de 2012), pgs. 1914-1928. IssN: 1097-0193. DOI: 10.1002/hbm.21333 (citado na pg. 30).

[Demirci et al. 2008] M. Fatih Demirci, Reinier H. van Leuken e Remco C. Veltkamp. "Indexing through laplacian spectra". en. Em: Computer Vision and Image Understanding. Similarity Matching in Computer Vision and Multimedia 110.3 (jun. de 2008), pgs. 312-325. ISSN: 1077-3142. DOI: 10.1016/j.cviu.2007.09.012. (Acesso em 23/09/2020) (citado na pg. 5).

[Di Martino et al. 2017] Adriana Di Martino et al. "Enhancing studies of the connectome in autism using the autism brain imaging data exchange II". Em: Scientific Data 4 (mar. de 2017). ISSN: 2052-4463. DOI: 10.1038/sdata.2017.10. URL: http: //www.ncbi.nlm.nih.gov/pmc/articles/PMC5349246/ (acesso em 16/09/2017) (citado na pg. 29).

[Drysdale et al. 2017] Andrew T. Drysdale et al. "Resting-state connectivity biomarkers define neurophysiological subtypes of depression”. eng. Em: Nature Medicine 23.1 (2017), pgs. 28-38. ISSN: 1546-170X. DOI: 10.1038/nm.4246 (citado nas pgs. 2, $28,48)$.

[Duku et al. 2013] Eric Duku et al. "Investigating the Measurement Properties of the Social Responsiveness Scale in Preschool Children with Autism Spectrum Disorders". en. Em: Fournal of Autism and Developmental Disorders 43.4 (abr. de 2013), pgs. 860-868. ISSN: 1573-3432. DOI: 10.1007 / s10803-012-1627-4. URL: https://doi.org/10.1007/s10803-012-1627-4 (acesso em 09/07/2020) (citado na pg. 49).

[Dumitriu, Pal et al. 2012] Ioana Dumitriu, Soumik Pal et al. "Sparse regular random graphs: spectral density and eigenvectors”. Em: The Annals of Probability 40.5 (2012), pgs. 2197-2235 (citado na pg. 16). 
[Elsabbagh et al. 2012] Mayada Elsabbagh et al. "Global Prevalence of Autism and Other Pervasive Developmental Disorders”. en. Em: Autism Research 5.3 (jun. de 2012), pgs. 160-179. ISSN: 1939-3806. DOI: 10 . 1002 / aur. 239. URL: http : / / onlinelibrary.wiley.com/doi/10.1002/aur.239/abstract (acesso em 04/05/2017) (citado na pg. 27).

[ERdös e Rényi 1959] Paul Erdös e Alfréd RÉnyi. "On random graphs, i”. Em: Publicationes Mathematicae (Debrecen) 6 (1959), pgs. 290-297 (citado na pg. 12).

[FARKas et al. 2001] Illés J FARKAS, Imre Derényi, Albert-László BarabÁsi e Tamas VicSEK. "Spectra of "real-world" graphs: beyond the semicircle law". Em: Physical Review E 64.2 (2001), pg. 026704 (citado nas pgs. 9, 13, 16).

[Fernandes et al. 2020] Orlando Fernandes et al. "How do you perceive threat? It's all in your pattern of brain activity". eng. Em: Brain Imaging and Behavior 14.6 (dez. de 2020), pgs. 2251-2266. ISSN: 1931-7565. DOI: 10.1007/s11682-019-00177-6 (citado na pg. 28).

[Fox e RAICHLE 2007] Michael D. Fox e Marcus E. Raichle. "Spontaneous fluctuations in brain activity observed with functional magnetic resonance imaging”. en. Em: Nature Reviews Neuroscience 8.9 (set. de 2007), pgs. 700-711. ISSN: 1471-0048. DOI: 10.1038/nrn2201. URL: https://www. nature.com/articles/nrn2201 (acesso em 28/01/2021) (citado nas pgs. 1, 28).

[Friston 2009] Karl J. Friston. "Modalities, Modes, and Models in Functional Neuroimaging”. en. Em: Science 326.5951 (out. de 2009), pgs. 399-403. ISSN: 0036-8075, 1095-9203. DOI: 10.1126/science.1174521. URL: https://science.sciencemag.org/ content/326/5951/399 (acesso em 28/01/2021) (citado na pg. 1).

[Fujiwara e Irie 2014] Yasuhiro Fujiwara e Go Irie. "Efficient label propagation". Em: International Conference on Machine Learning. 2014, pgs. 784-792 (citado na pg. 5).

[Gates e Ahn 2019] Alexander J. Gates e Yong-Yeol Ahn. "CluSim: a python package for calculating clustering similarity”. en. Em: Journal of Open Source Software 4.35 (mar. de 2019), pg. 1264. ISSN: 2475-9066. DOI: 10.21105/joss.01264. (Acesso em 02/09/2020) (citado na pg. 16).

[Gioia et al. 2018] Gerard A. Gioia, Peter K. IsQuith e Robert M. Roth. "Behavior Rating Inventory for Executive Function”. en. Em: Encyclopedia of Clinical Neuropsychology. Ed. por Jeffrey S. Kreutzer, John DeLuca e Bruce CAPLAN. Cham: Springer International Publishing, 2018, pgs. 532-538. ISBN: 9783319571119. DOI: 10.1007/978-3-319-57111-9_1881. uRL: https://doi.org/10.1007/978-3-319-571119_1881 (acesso em 23/10/2020) (citado nas pgs. 28, 32). 
REFERÊNCIAS

[Goldani et al. 2014] Andre A. S. Goldani, Susan R. Downs, Felicia Widjaja, Brittany Lawton e Robert L. Hendren. "Biomarkers in Autism". English. Em: Frontiers in Psychiatry 5 (2014). ISSN: 1664-0640. DOI: 10.3389/fpsyt.2014.00100. URL: https:// www.frontiersin.org/articles/10.3389/fpsyt.2014.00100/full (acesso em 09/06/2020) (citado na pg. 28).

[Granader et al. 2014] Yael Granader et al. "Characterizing the factor structure of parent reported executive function in autism spectrum disorders: the impact of cognitive inflexibility”. eng. Em: Journal of Autism and Developmental Disorders 44.12 (dez. de 2014), pgs. 3056-3062. ISSN: 1573-3432. Dor: 10.1007/s10803-0142169-8 (citado na pg. 49).

[Havdahl et al. 2016] K. Alexandra Havdahl, Stephen von Tetzchner, Marisela HuERTA, Catherine LORD e Somer L. Bishop. "Utility of the Child Behavior Checklist as a Screener for Autism Spectrum Disorder”. Em: Autism research : official journal of the International Society for Autism Research 9.1 (jan. de 2016), pgs. 33-42. IssN: 1939-3792. DOI: 10.1002/aur.1515. URL: https://www.ncbi.nlm.nih.gov/pmc/ articles/PMC4939629/ (acesso em 19/06/2020) (citado nas pgs. 29, 49).

[Hotelling 1935] H. Hotelling. "The most predictable criterion". English. Em: fournal of Educational Psychology 26.2 (1935), pgs. 139-142. IssN: 1939-2176 0022-0663. DOI: 10.1037/h0058165 (citado na pg. 34).

[INSEL et al. 2010] Thomas InsEl et al. "Research Domain Criteria (RDoC): Toward a New Classification Framework for Research on Mental Disorders". Em: American fournal of Psychiatry 167.7 (jul. de 2010), pgs. 748-751. ISSN: 0002-953X. DOI: 10.1176/appi.ajp.2010.09091379. uRL: https://ajp.psychiatryonline.org/doi/full/10. 1176/appi.ajp.2010.09091379 (acesso em 10/06/2020) (citado na pg. 27).

[Kapur et al. 2012] S. Kapur, A. G. Phillips e T. R. Insel. "Why has it taken so long for biological psychiatry to develop clinical tests and what to do about it?" eng. Em: Molecular Psychiatry 17.12 (dez. de 2012), pgs. 1174-1179. ISsN: 1476-5578. DOI: 10.1038/mp.2012.105 (citado na pg. 27).

[Kenworthy et al. 2008] Lauren Kenworthy, Benjamin E. Yerys, Laura Gutermuth Anthony e Gregory L. WAllace. "Understanding executive control in autism spectrum disorders in the lab and in the real world". Em: Neuropsychology review 18.4 (dez. de 2008), pgs. 320-338. ISSN: 1040-7308. DOI: 10.1007/s11065-008-9077-7. URL: https://www.ncbi.nlm.nih.gov/pmc/articles/PMC2856078/ (acesso em 19/06/2020) (citado na pg. 29).

[KING et al. 2019] Jace B. KING et al. "Generalizability and reproducibility of functional connectivity in autism”. Em: Molecular Autism 10.1 (jun. de 2019), pg. 27. ISSN: 2040-2392. DOI: 10.1186/s13229-019-0273-5. URL: https://doi.org/10.1186/s13229019-0273-5 (acesso em 09/06/2020) (citado na pg. 28). 
[LAnge et al. 2014] Siemon de Lange, Marcel de Reus e Martijn Van Den Heuvel. "The Laplacian spectrum of neural networks”. English. Em: Frontiers in Computational Neuroscience 7 (2014). ISSN: 1662-5188. DOI: 10.3389/fncom.2013.00189. (Acesso em 21/08/2020) (citado na pg. 13).

[LÊ CaO et al. 2008] Kim-Anh LÊ CaO, Debra Rossouw, Christèle Robert-Granié e Philippe BESSE. "A sparse PLS for variable selection when integrating omics data”. eng. Em: Statistical Applications in Genetics and Molecular Biology 7.1 (2008), Article 35. ISSN: 1544-6115. DOI: 10.2202/1544-6115.1390 (citado na pg. 34).

[Levandowsky e Winter 1971] Michael Levandowsky e David Winter. "Distance between Sets”. en. Em: Nature 234.5323 (nov. de 1971), pgs. 34-35. IssN: 1476-4687. DOI: 10.1038/234034a0. (Acesso em 03/11/2018) (citado na pg. 16).

[Lloyd 1982] Stuart Lloyd. "Least squares quantization in pcm". Em: IEEE transactions on information theory 28.2 (1982), pgs. 129-137 (citado nas pgs. 1, 5).

[Lombardo et al. 2019] Michael V. Lombardo, Meng-Chuan Lai e Simon BaronCoHEN. "Big data approaches to decomposing heterogeneity across the autism spectrum”. en. Em: Molecular Psychiatry 24.10 (out. de 2019), pgs. 1435-1450. ISSN: 1476-5578. DOI: 10.1038/s41380-018-0321-0. URL: https://www. nature.com/ articles/s41380-018-0321-0 (acesso em 10/06/2020) (citado na pg. 27).

[Luxburg 2007] Ulrike von Luxburg. “A tutorial on spectral clustering”. en. Em: Statistics and Computing 17.4 (dez. de 2007), pgs. 395-416. ISSN: 1573-1375. DOI: 10.1007/s11222-007-9033-z. (Acesso em 09/11/2020) (citado na pg. 5).

[MAckey 2008] Lester Mackey. "Deflation Methods for Sparse PCA". en. Em: Advances in Neural Information Processing Systems 21 (2008), pgs. 1017-1024. URL: https: //papers.nips.cc/paper/2008/hash/85d8ce590ad8981ca2c8286f79f59954-Abstract. html (acesso em 13/01/2021) (citado na pg. 41).

[MAcQueEn et al. 1967] James MAcQueEn et al. "Some methods for classification and analysis of multivariate observations". Em: Proceedings of the fifth Berkeley symposium on mathematical statistics and probability. Vol. 1. 14. Oakland, CA, USA. 1967, pgs. 281-297 (citado nas pgs. 1, 5).

[Mash et al. 2018] Lisa E. Mash, Maya A. Reiter, Annika C. Linke, Jeanne Townsend e Ralph-Axel MüLLER. "Multimodal approaches to functional connectivity in autism spectrum disorders: An integrative perspective”. en. Em: Developmental Neurobiology 78.5 (2018), pgs. 456-473. ISSN: 1932-846X. DOI: 10.1002/dneu.22570. URL: https:// onlinelibrary.wiley.com/doi/abs/10.1002/dneu.22570 (acesso em 09/06/2020) (citado na pg. 28).

[Maslov e Sneppen 2002] Sergei Maslov e Kim Sneppen. "Specificity and Stability in Topology of Protein Networks”. en. Em: Science 296.5569 (mai. de 2002), pgs. 910913. ISSN: 0036-8075, 1095-9203. DOI: $10.1126 /$ science.1065103. (Acesso em 21/08/2020) (citado na pg. 13). 
REFERÊNCIAS

[Maximo et al. 2014] Jose O. Maximo, Elyse J. Cadena e Rajesh K. Kana. "The implications of brain connectivity in the neuropsychology of autism". eng. Em: Neuropsychology Review 24.1 (mar. de 2014), pgs. 16-31. ISSN: 1573-6660. DOI: 10.1007/s11065-014-9250-0 (citado na pg. 28).

[McKay 1981] Brendan D McKay. "The expected eigenvalue distribution of a large regular graph”. Em: Linear Algebra and its Applications 40 (1981), pgs. 203-216 (citado na pg. 16).

[Mihalik, Ferreira, Moutoussis et al. 2020] Agoston Mihalik, Fabio S. Ferreira, Michael Moutoussis et al. "Multiple Holdouts With Stability: Improving the Generalizability of Machine Learning Analyses of Brain-Behavior Relationships". English. Em: Biological Psychiatry 87.4 (fev. de 2020), pgs. 368-376. IssN: 00063223, 1873-2402. DOI: $10.1016 / \mathrm{j}$. biopsych.2019.12.001. URL: https:// www . biologicalpsychiatryjournal.com/article/S0006-3223(19)31918-3/abstract (acesso em 05/06/2020) (citado nas pgs. 2, 28, 34, 36, 51).

[Mihalik, Ferreira, Rosa et al. 2019] Agoston Mihalik, Fabio S. Ferreira, Maria J. Rosa et al. "Brain-behaviour modes of covariation in healthy and clinically depressed young people”. en. Em: Scientific Reports 9.1 (ago. de 2019), pg. 11536. ISSN: 2045-2322. DOI: 10.1038/s41598-019-47277-3. URL: https://www.nature.com/ articles/s41598-019-47277-3 (acesso em 29/07/2020) (citado nas pgs. 2, 48).

[Monteiro, Rao, Ashburner et al. 2016] João M. Monteiro, Anil Rao, John AshburNER, John Shawe-TaYlor e Janaina Mourão-MirandA. "Leveraging Clinical Data to Enhance Localization of Brain Atrophy”. en. Em: Machine Learning and Interpretation in Neuroimaging. Ed. por Irina RISH et al. Lecture Notes in Computer Science. Cham: Springer International Publishing, 2016, pgs. 60-68. ISBN: 9783319451749. DOI: 10.1007/978-3-319-45174-9_7 (citado na pg. 41).

[Monteiro, Rao, Shawe-Taylor et al. 2016] João M. Monteiro, Anil Rao, John Shawe-Taylor e Janaina Mourão-Miranda. "A multiple hold-out framework for Sparse Partial Least Squares”. Em: Fournal of Neuroscience Methods 271 (set. de 2016), pgs. 182-194. ISSN: 0165-0270. DOI: 10.1016/j.jneumeth . 2016.06.011. URL: https://www.ncbi.nlm.nih.gov/ pmc/articles/PMC5012894/ (acesso em 05/06/2020) (citado nas pgs. 2, 36, 40).

[D. A. Moser et al. 2018] D. A. Moser et al. "An integrated brain-behavior model for working memory”. en. Em: Molecular Psychiatry 23.10 (out. de 2018), pgs. 19741980. ISSN: 1476-5578. DOI: 10.1038/mp.2017.247. URL: https://www.nature.com/ articles/mp2017247 (acesso em 04/02/2021) (citado na pg. 35).

[Dominik A. Moser et al. 2018] Dominik A. Moser et al. "Multivariate Associations Among Behavioral, Clinical, and Multimodal Imaging Phenotypes in Patients With Psychosis". en. Em: FAMA Psychiatry 75.4 (abr. de 2018), pgs. 386-395. IssN: 2168-622X. DOI: 10.1001/jamapsychiatry.2017.4741. URL: https://jamanetwork. com/journals/jamapsychiatry/fullarticle/2673930 (acesso em 29/07/2020) (citado na pg. 48). 
[Mottron e BzDok 2020] Laurent Mottron e Danilo Bzdok. "Autism spectrum heterogeneity: fact or artifact?” en. Em: Molecular Psychiatry (abr. de 2020), pgs. 1-8. ISSN: 1476-5578. DOI: 10.1038/s41380-020-0748-y. URL: https://www.nature.com/ articles/s41380-020-0748-y (acesso em 10/06/2020) (citado na pg. 27).

[NAgasaka et al. 2011] Yasuo Nagasaka, Kentaro Shimoda e Naotaka Fujir. "Multidimensional recording (MDR) and data sharing: an ecological open research and educational platform for neuroscience”. eng. Em: PLoS ONE 6.7 (2011), e22561. ISSN: 1932-6203. DOI: 10.1371/journal.pone.0022561 (citado nas pgs. 2, 15).

[O’Reilly et al. 2017] Christian O’Reilly, John D. Lewis e Mayada Elsabbagh. "Is functional brain connectivity atypical in autism? A systematic review of EEG and MEG studies”. Em: PLoS ONE 12.5 (2017). ISSN: 1932-6203(Electronic) (citado na pg. 28).

[Oliveira et al. 2019] Leticia de Oliveira et al. "Predicting Bipolar Disorder Risk Factors in Distressed Young Adults From Patterns of Brain Activation to Reward: A Machine Learning Approach”. eng. Em: Biological Psychiatry. Cognitive Neuroscience and Neuroimaging 4.8 (ago. de 2019), pgs. 726-733. ISSN: 2451-9030. DOI: 10.1016/j.bpsc.2019.04.005 (citado na pg. 28).

[Organization 2004] World Health Organization. International Statistical Classification of Diseases and Related Health Problems. en. Google-Books-ID: Tw5eAtsatiUC. World Health Organization, 2004. ISBN: 9789241546492 (citado na pg. 27).

[Penrose 1999] Mathew D Penrose. "On k-connectivity for a geometric random graph". Em: Random Structures \& Algorithms 15.2 (1999), pgs. 145-164 (citado na pg. 12).

[Portugal et al. 2019] Liana C. L. Portugal et al. "Predicting anxiety from wholebrain activity patterns to emotional faces in young adults: a machine learning approach". eng. Em: NeuroImage. Clinical 23 (2019), pg. 101813. ISSN: 2213-1582. DOI: 10.1016/ j.nicl.2019.101813 (citado na pg. 28).

[Power et al. 2012] Jonathan D. Power, Kelly A. BArnes, Abraham Z. Snyder, Bradley L. Schlaggar e Steven E. Petersen. "Spurious but systematic correlations in functional connectivity MRI networks arise from subject motion”. eng. Em: NeuroImage 59.3 (fev. de 2012), pgs. 2142-2154. IssN: 1095-9572. DOI: 10.1016/j. neuroimage.2011.10.018 (citado na pg. 30).

[Pratt e Hall 2018] Judith Pratt e Jeremy Hall. "Biomarkers in Neuropsychiatry: A Prospect for the Twenty-First Century?” en. Em: Biomarkers in Psychiatry. Ed. por Judith Pratt e Jeremy Hall. Current Topics in Behavioral Neurosciences. Cham: Springer International Publishing, 2018, pgs. 3-10. ISBN: 9783319996424. DOI: 10.1007/7854_2018_58. URL: https://doi.org/10.1007/7854_2018_58 (acesso em 12/06/2020) (citado na pg. 28). 
REFERÊNCIAS

[Ramos et al. 2019] Taiane Coelho Ramos, Joana Bisol Balardin, João Ricardo Sato e André Fujita. "Abnormal Cortico-Cerebellar Functional Connectivity in Autism Spectrum Disorder”. English. Em: Frontiers in Systems Neuroscience 12 (2019). ISSN: 1662-5137. DOI: 10.3389/fnsys.2018.00074. URL: https://www.frontiersin.org/ articles/10.3389/fnsys.2018.00074/full (acesso em 05/06/2020) (citado na pg. 48).

[RosA et al. 2015] Maria J. Rosa et al. "Estimating multivariate similarity between neuroimaging datasets with sparse canonical correlation analysis: an application to perfusion imaging”. English. Em: Frontiers in Neuroscience 9 (2015). ISSN: 1662453X. DOI: 10.3389/fnins.2015.00366. URL: https://www.frontiersin.org/articles/ 10.3389/fnins.2015.00366/full (acesso em 04/02/2021) (citado na pg. 35).

[Rousseeuw 1987] Peter J Rousseeuw. "Silhouettes: a graphical aid to the interpretation and validation of cluster analysis". Em: Fournal of computational and applied mathematics 20 (1987), pgs. 53-65 (citado nas pgs. 8, 9).

[SATo et al. 2016] João Ricardo SATo, Joana BAlardin, Maciel Calebe VidAl e André FujITA. "Identification of segregated regions in the functional brain connectome of autistic patients by a combination of fuzzy spectral clustering and entropy analysis". Em: Journal of Psychiatry \& Neuroscience : JPN 41.2 (mar. de 2016), pgs. 124-132. ISSN: 1180-4882. DOI: 10.1503/jpn.140364. URL: https://www.ncbi. nlm.nih.gov/pmc/articles/ PMC4764481/ (acesso em 05/06/2020) (citado nas pgs. 43,46$)$.

[Singh e Rose 2009] Ilina Singh e Nikolas Rose. "Biomarkers in psychiatry". en. Em: Nature 460.7252 (jul. de 2009), pgs. 202-207. ISSN: 1476-4687. DOI: 10.1038/460202a. URL: https://www.nature.com/articles/460202a (acesso em 12/06/2020) (citado na pg. 28).

[Siqueira Santos et al. 2016] Suzana de Siqueira Santos, Daniel Yasumasa TAKAHASHI, João Ricardo Sato, Carlos Eduardo Ferreira e André Fujita. "Statistical methods in graphs: parameter estimation, model selection, and hypothesis test". Em: Mathematical Foundations and Applications of Graph Entropy (2016), pg. 98 (citado nas pgs. 7, 10).

[S. M. Smith 2012] Stephen M. Smith. “The future of FMRI connectivity”. eng. Em: NeuroImage 62.2 (ago. de 2012), pgs. 1257-1266. IsSN: 1095-9572. DOI: 10.1016/j. neuroimage.2012.01.022 (citado na pg. 1).

[S. Smith et al.2015] Stephen Sмiтh et al. "A positive-negative mode of population covariation links brain connectivity, demographics and behavior”. Em: Nature neuroscience 18.11 (nov. de 2015), pgs. 1565-1567. ISSN: 1097-6256. DOI: 10.1038/ nn.4125. URL: https://www.ncbi.nlm.nih.gov/pmc/articles/PMC4625579/ (acesso em 05/06/2020) (citado nas pgs. 2, 28, 48). 
[Spielman e Teng 2007] Daniel A. Spielman e Shang-Hua Teng. "Spectral partitioning works: Planar graphs and finite element meshes". en. Em: Linear Algebra and its Applications. Special Issue in honor of Miroslav Fiedler 421.2 (mar. de 2007), pgs. 284-305. ISSN: 0024-3795. DOI: 10.1016/j.laa.2006.07.020. (Acesso em 09/11/2020) (citado na pg. 5).

[TAkahashi et al. 2012] Daniel Yasumasa TAkahashi, Joao Ricardo SAto, Carlos Eduardo Ferreira e André Fujita. "Discriminating different classes of biological networks by analyzing the graphs spectra distribution”. Em: PLoS ONE 7.12 (2012), e49949 (citado nas pgs. 7, 10, 11, 23).

[TANg et al. 2020] Siyi TANg et al. "Reconciling Dimensional and Categorical Models of Autism Heterogeneity: A Brain Connectomics and Behavioral Study". English. Em: Biological Psychiatry 87.12 (jun. de 2020), pgs. 1071-1082. ISSN: 00063223, 1873-2402. DoI: 10.1016 / j. biopsych. 2019 .11.009. uRL: https://www. biologicalpsychiatryjournal.com/article/S0006-3223(19)31859-1/abstract (acesso em 29/07/2020) (citado nas pgs. 27, 48).

[R. Tibshirani e Walther 2005] Robert Tibshirani e Guenther Walther. "Cluster Validation by Prediction Strength". Em: Journal of Computational and Graphical Statistics 14.3 (set. de 2005), pgs. 511-528. ISSN: 1061-8600. DOI: 10.1198 / 106186005X59243. (Acesso em 31/08/2020) (citado na pg. 16).

[Tran et al. 2013] Linh V Tran, Van H Vu e Ke Wang. "Sparse random graphs: eigenvalues and eigenvectors”. Em: Random Structures \& Algorithms 42.1 (2013), pgs. 110-134 (citado nas pgs. 9, 16).

[Ugander e Backstrom 2013] Johan Ugander e Lars Backstrom. "Balanced label propagation for partitioning massive graphs". Em: Proceedings of the sixth ACM international conference on Web search and data mining. WSDM '13. New York, NY, USA: Association for Computing Machinery, fev. de 2013, pgs. 507-516. IsBN: 9781450318693. DOI: 10.1145/2433396.2433461. (Acesso em 09/11/2020) (citado na pg. 5).

[UhlhaAs e Singer 2012] Peter J. UhlhaAs e Wolf Singer. "Neuronal Dynamics and Neuropsychiatric Disorders: Toward a Translational Paradigm for Dysfunctional Large-Scale Networks”. en. Em: Neuron 75.6 (set. de 2012), pgs. 963-980. IssN: 0896-6273. DOI: 10.1016/j.neuron.2012.09.004. URL: http://www.sciencedirect. com/science/article/pii/S0896627312008112 (acesso em 28/01/2021) (citado na pg. 1).

[Vasa et al. 2016] Roma A. VAsA, Stewart H. Mostofsky e Joshua B. Ewen. "The Disrupted Connectivity Hypothesis of Autism Spectrum Disorders: Time for the Next Phase in Research”. en. Em: Biological Psychiatry: Cognitive Neuroscience and Neuroimaging. Brain Connectivity in Psychopathology 1.3 (mai. de 2016), pgs. 245-252. ISSN: 2451-9022. DOI: $10.1016 / \mathrm{j}$. bpsc. 2016.02.003. URL: http: // www. sciencedirect.com/science/ article / pii / S2451902216300015 (acesso em 09/06/2020) (citado na pg. 28). 
REFERÊNCIAS

[WANG et al. 2019] Kai WANG et al. "Altered social cognition and connectivity of default mode networks in the co-occurrence of autistic spectrum disorder and attention deficit hyperactivity disorder”. eng. Em: The Australian and New Zealand fournal of Psychiatry 53.8 (2019), pgs. 760-771. ISSN: 1440-1614. DOI: 10.1177 / 0004867419836031 (citado na pg. 48).

[Watts e Strogatz 1998] Duncan J Watts e Steven H Strogatz. "Collective dynamics of 'small-world'networks". Em: Nature 393.6684 (1998), pg. 440 (citado nas pgs. 12, 13).

[Wh et al. 2018] Lee Wh, Moser DA, Ing A, Doucet Ge e Frangou S. "Behavioral and Health Correlates of Resting-State Metastability in the Human Connectome Project." English. Em: Brain Topography 32.1 (ago. de 2018), pgs. 80-86. IssN: 0896-0267, 1573-6792. DOI: 10.1007/s10548-018-0672-5. uRL: https://europepmc.org/article/ pmc/pmc6326990 (acesso em 04/02/2021) (citado na pg. 35).

[Wills e Meyer 2020] Peter Wills e François G. Meyer. "Metrics for graph comparison: A practitioner's guide”. Em: PLoS ONE 15.2 (fev. de 2020). ISSN: 1932-6203. DOI: 10.1371/journal.pone.0228728. (Acesso em 12/08/2020) (citado na pg. 5).

[Wilson e P. Zhu 2008] Richard C. Wilson e Ping Zhu. "A study of graph spectra for comparing graphs and trees”. en. Em: Pattern Recognition 41.9 (set. de 2008), pgs. 2833-2841. ISSN: 0031-3203. DOI: 10.1016/j.patcog.2008.03.011. (Acesso em 17/09/2020) (citado na pg. 5).

[Witten e R. J. Tibshirani 2009] Daniela M Witten e Robert J. Tibshirani. "Extensions of Sparse Canonical Correlation Analysis with Applications to Genomic Data”. Em: Statistical Applications in Genetics and Molecular Biology 8.1 (jun. de 2009). ISSN: 1544-6115. DOI: 10.2202/1544-6115.1470. URL: https://www.ncbi.nlm. nih.gov/pmc/articles/PMC2861323/ (acesso em 03/02/2021) (citado na pg. 35).

[C. H. XIA et al. 2018] Cedric Huchuan Xia et al. "Linked dimensions of psychopathology and connectivity in functional brain networks". en. Em: Nature Communications 9.1 (ago. de 2018), pg. 3003. ISSN: 2041-1723. DOI: 10.1038/s41467-01805317-y. URL: https://www.nature.com/articles/s41467-018-05317-y (acesso em 29/07/2020) (citado na pg. 48).

[M. XIA et al. 2013] Mingrui Xia, Jinhui Wang e Yong He. "BrainNet Viewer: A Network Visualization Tool for Human Brain Connectomics”. Em: PLoS ONE 8.7 (jul. de 2013). ISSN: 1932-6203. DOI: 10.1371 / journal . pone.0068910. URL: https : / / www . ncbi . nlm . nih .gov / pmc/articles / PMC3701683/ (acesso em 05/06/2020) (citado na pg. 43).

[Yahata et al. 2017] Noriaki Yahata, Kiyoto Kasai e Mitsuo Kawato. "Computational neuroscience approach to biomarkers and treatments for mental disorders”. en. Em: Psychiatry and Clinical Neurosciences 71.4 (2017), pgs. 215-237. IssN: 14401819. DOI: $10.1111 /$ pcn.12502. uRL: https://onlinelibrary.wiley.com/doi/abs/10. 1111/pcn.12502 (acesso em 12/06/2020) (citado na pg. 28). 
[Yanagawa et al. 2013] Toru Yanagawa, Zenas C. Chao, Naomi Hasegawa e Naotaka FujII. "Large-Scale Information Flow in Conscious and Unconscious States: an ECoG Study in Monkeys”. en. Em: PLoS ONE 8.11 (nov. de 2013), e80845. IssN: 1932-6203. DOI: 10.1371/journal.pone.0080845. (Acesso em 26/09/2018) (citado na pg. 15).

[X. Zhu e Ghahramani 2002] Xiaojin Zhu e Zoubin Ghahramani. "Learning from labeled and unlabeled data with label propagation”. Em: (2002) (citado na pg. 5).

[Zou e Hastie 2005] Hui Zou e Trevor Hastie. "Regularization and variable selection via the elastic net”. en. Em: fournal of the Royal Statistical Society: Series B (Statistical Methodology) 67.2 (2005), pgs. 301-320. ISSN: 1467-9868. DOI: https: //doi.org/10.1111/j.1467-9868.2005.00503.x. URL: https://rss.onlinelibrary.wiley. com/doi/abs/10.1111/j.1467-9868.2005.00503.x (acesso em 04/02/2021) (citado na pg. 35). 